\title{
Testing a Criterion for Dislocation Nucleation using Molecular Dynamics Simulations of Nano-Indentation
}

by

\section{YanJuan Zhao,} B.Tech. Beijing Institute of Technology, 1995

\begin{abstract}
A thesis submitted to the Faculty of Graduate Studies and Research in partial fulfillment of the requirements for the degree of

\section{Master of Applied Science}

Ottawa-Carleton Institute for Mechanical and Aerospace Engineering

\author{
Department of Mechanical and Aerospace Engineering \\ Carleton University \\ Ottawa, Ontario \\ Canada
}

September 2005

(C) Copyright by YanJuan Zhao

2005 


$\begin{array}{ll}\begin{array}{l}\text { Library and } \\ \text { Archives Canada }\end{array} & \begin{array}{l}\text { Bibliothèque et } \\ \text { Archives Canada }\end{array} \\ \begin{array}{l}\text { Published Heritage } \\ \text { Branch }\end{array} & \begin{array}{l}\text { Direction du } \\ \text { Patrimoine de l'édition }\end{array} \\ \begin{array}{l}\text { 395 Wellington Street } \\ \text { Ottawa ON K1A ON4 }\end{array} & \begin{array}{l}\text { 395, rue Wellington } \\ \text { Ottawa ON K1A ON4 } \\ \text { Canada }\end{array} \\ \end{array}$

Your file Votre référence ISBN: 0-494-10094-X Our file Notre référence ISBN: 0-494-10094-X

NOTICE:

The author has granted a nonexclusive license allowing Library and Archives Canada to reproduce, publish, archive, preserve, conserve, communicate to the public by telecommunication or on the Internet, loan, distribute and sell theses worldwide, for commercial or noncommercial purposes, in microform, paper, electronic and/or any other formats.

The author retains copyright ownership and moral rights in this thesis. Neither the thesis nor substantial extracts from it may be printed or otherwise reproduced without the author's permission.
AVIS:

L'auteur a accordé une licence non exclusive permettant à la Bibliothèque et Archives Canada de reproduire, publier, archiver, sauvegarder, conserver, transmettre au public par télécommunication ou par l'Internet, prêter, distribuer et vendre des thèses partout dans le monde, à des fins commerciales ou autres, sur support microforme, papier, électronique et/ou autres formats.

L'auteur conserve la propriété du droit d'auteur et des droits moraux qui protège cette thèse. $\mathrm{Ni}$ la thèse ni des extraits substantiels de celle-ci ne doivent être imprimés ou autrement reproduits sans son autorisation.
In compliance with the Canadian

Privacy Act some supporting forms may have been removed from this thesis.

While these forms may be included in the document page count, their removal does not represent any loss of content from the thesis.
Conformément à la loi canadienne sur la protection de la vie privée, quelques formulaires secondaires ont été enlevés de cette thèse.

Bien que ces formulaires aient inclus dans la pagination, il n'y aura aucun contenu manquant.

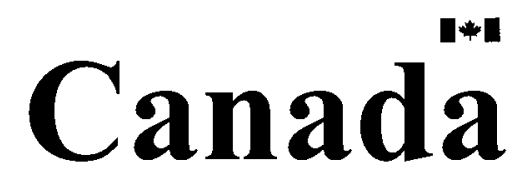


The undersigned recommend to the Faculty of Graduate Studies and Research acceptance of the thesis

Testing a Criterion for Dislocation Nucleation using Molecular Dynamics Simulations of Nano-Indentation

submitted by

YanJuan Zhao, B.Tech.

in partial fulfilment of the requirements for the degree of Master of Applied Science

Thesis Supervisor

Chair, Dept. of Mechanical and Aerospace Engineering

Carleton University

September 12, 2005 


\section{Abstract}

Dislocations are the main lattice defects responsible for the strength and ductility of crystalline solids. Understanding how a new dislocation generates is an essential aspect of crystal defect physics. The commonly held notion of a nucleation criterion based on Schmid's Law (whereby the dislocation will nucleate when the resolved stress acting on it reaches a critical value), is incorrect to predict the dislocation nucleation for some crystal structures. Here, a new nucleation criterion is tested based on stress gradients of a crystalline solid undergoing deformation, and demonstrate the criterion's ability to correctly predict dislocation nucleation via direct atomistic simulations.

Three-dimensional MD (Molecular Dynamics) is used to simulate dislocation nucleation with indentation. The atoms are modeled by empirical interatomic potentials based on the EAM (Embedded Atom Method). The indenter was modeled as a fictitious atom with a specific radius, a repulsive interatomic potential between the indenter and the atoms of the metal is also introduced. A material value for nucleation criterion, $\mathrm{N}_{m, l}$, was computed at every atom in the crystal for every possible dislocation (defined by Burgers Vector and Slip plane) according to the stress gradients. Comparing with the distribution of real dislocations nucleated in the crystal, current MD simulations provide a good agreement between the criterion predictions and actual simulation results. 


\section{Acknowledgments}

This thesis is an outcome of almost two years of work whereby I have been accompanied and supported by many people. It is a pleasant aspect that I have now the opportunity to express my gratitude towards all of them.

I would like to start by thanking my thesis supervisor, Professor Ronald E. Miller for his time, generosity, patience, and insight. He has inspired me in the most important moments of making the right decisions. His guidance is highly appreciated.

I am also thankful to my colleagues who have been supportive and encouraging, Dr. Denis Saraev, Ishraq Shabib, Dr. Behrouz Shiari.

Finally, but not least importantly, I would like to thank my family for their innumerable sacrifices, love, support, continuous inspiration and faith in me. 
To my family

iii

Reproduced with permission of the copyright owner. Further reproduction prohibited without permission. 


\title{
Contents
}

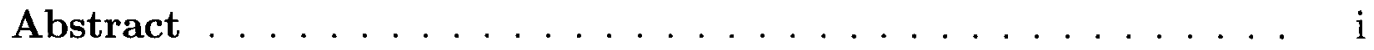

Acknowledgements ................... ii

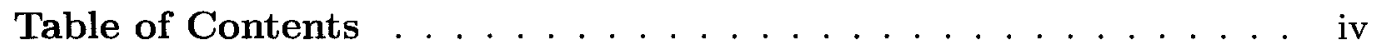

List of Figures ..................... . . vi

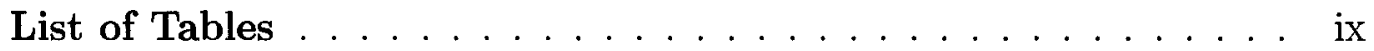

Nomenclature . . . . . . . . . . . . . . . . $\mathrm{x}$
\end{abstract}

1 Introduction 1

1.1 Criterion of Dislocation Nucleation . . . . . . . . . . . . 1

1.1.1 The previous nucleation criteria . . . . . . . . . . 2

1.1.2 Description of the "Acharya/Miller" criterion . . . . . . . . 3

1.2 Overview of the thesis $\ldots \ldots \ldots \ldots$

2 Background and Literature Review $\quad 7$

2.1 The face centered cubic $(\mathrm{fcc}) \ldots \ldots \ldots \ldots$

2.1 .1 fcc slip system . . . . . . . . . . . . 8

2.2 Dislocations in fcc crystal structures f . . . . . . . . . . . 9

2.2 .1 Edge dislocation $\ldots \ldots \ldots \ldots$

2.2 .2 Screw dislocation . . . . . . . . . . . . . . 12

2.2 .3 Burgers vector . . . . . . . . . . . . . . . . 12

2.2 .4 Mixed dislocation . . . . . . . . . . . . . . . 16

2.2.5 Stacking faults \& Shockley partial dislocations . . . . . . . . 17 
2.3 Previous studies of Dislocation nucleation $\ldots \ldots \ldots$. . . . . . . . 21

2.3 .1 Nanoindentation method . . . . . . . . . . . . . . 21

2.3.2 Computational approach . . . . . . . . . . . . . 22

2.3.3 Experimental approach . . . . . . . . . . . . . . . . 28

2.4 Criteria of dislocation nucleation . . . . . . . . . . . 29

2.4.1 "Schmid-like" criterion . . . . . . . . . . . . . . . 30

$2.4 .2 " \wedge \ldots "$ criterion $\ldots \ldots \ldots \ldots \ldots \ldots$

2.4.3 "Acharya/Miller" criterion . . . . . . . . . . . . 33

3 Simulation tools and computational procedure 36

3.1 Simulation method . . . . . . . . . . . . . . 36

3.1.1 Description of Nano-indentation modeling . . . . . . . . 36

3.2 Testing of the "Acharya/Miller" criterion . . . . . . . . . . . . . 40

3.2.1 Potential between the indenter and its neighbor atoms . . . 40

3.2.2 Calculation of atomic level stresses . . . . . . . . . . . . 41

3.2.3 Computation of stress gradient with LOPI method . . . . . . 44

3.2 .4 Calculating $\mathrm{N}_{m, l} \ldots \ldots \ldots \ldots 6 \ldots$

3.3 Method to determine actual defect nucleation $\ldots \ldots \ldots$. . . . . . 63

3.3.1 Identifying the event of dislocation nucleation . . . . . . . 63

3.3.2 The indication of Burgers vector: Slip vector . . . . . . . . . . 63

4 Results and Discussion $\quad 67$

4.1 Pictorial representation of results . . . . . . . . . . . . 67

$4.1 .1 \mathrm{Cu}(001)$ model . . . . . . . . . . . . . . . 68

5 Conclusion $\quad 86$ 


\section{List of Figures}

1.1 Diagram of indentation model . . . . . . . . . . . . . 5

2.1 FCC structure for metals . . . . . . . . . . . . . . . 8

2.2 The $\{111\}$ planes of fcc . . . . . . . . . . . . . 9

2.3 Formation of a pure edge dislocation . . . . . . . . . . . . . 10

2.4 Illustration of edge dislocation . . . . . . . . . . . . . . . . 11

2.5 Slip of an edge dislocation . . . . . . . . . . . . . . . 11

2.6 Dislocation motion leading to plastic deformation . . . . . . . . 12

2.7 Screw dislocation line . . . . . . . . . . . . . . . 13

2.8 Burgers circuit of edge dislocation . . . . . . . . . . . . . . . 14

2.9 Burgers circuit of screw dislocation . . . . . . . . . . . . 15

2.10 Mixed dislocation . . . . . . . . . . . . . 16

2.11 Formation of Shockley partial dislocations . . . . . . . . . . 17

2.12 Burgers vectors of Shockley partial dislocation . . . . . . . . . . . 19

2.13 Formation of Shockley partial dislocations . . . . . . . . . . . . . 19

2.14 TEM graph of dislocations f . . . . . . . . . . . . . 20

2.15 Cathodo-luminescence imaging of dislocation loops . . . . . . . . 30

2.16 Definition of the resolved shear stress . . . . . . . . . . . . 31

2.17 The $\wedge_{\min }$ criterion . . . . . . . . . . . . . 32

2.18 Dislocation nucleation . . . . . . . . . . . . . . . 34

2.19 Predictions of dislocation nucleation . . . . . . . . . . . 35

vi 
3.1 Periodic boundary condition . . . . . . . . . . . . . 38

3.2 Atomic configuration of $(001)$ model . . . . . . . . . . . . . . 39

3.3 Traction definition . . . . . . . . . . . . . . . 44

3.4 least-square approximation . . . . . . . . . . . . . . . . . 48

3.5 Multiquadrics interpolation . . . . . . . . . . . . . 50

3.6 Distribution of Hertz stress . . . . . . . . . . . . . . . . 52

3.7 Stress gradient comparison . . . . . . . . . . . . . 56

$3.8 \mathrm{~N}_{m, l}$ vs. depth curves for $0 \leq x, y \leq 1 \ldots \ldots \ldots$

$3.9 \mathrm{~N}_{m, l}$ vs. depth curves for $4 \leq x, y \leq 5 \ldots \ldots \ldots \ldots$

$3.10 \mathrm{~N}_{m, l}$ vs. depth curves for $16 \leq x, y \leq 17 \ldots \ldots \ldots$

$3.11 \mathrm{~N}_{m, l}$ vs. depth curves for $-1 \leq x, y \leq 0 \ldots \ldots \ldots$

3.12 Burgers vector on slip plane . . . . . . . . . . . . . . . 62

3.13 Prediction of the "Acharya/Miller" criterion . . . . . . . . . . . . . 64

3.14 Definition of $\operatorname{slip}$ vector $\ldots \ldots \ldots \ldots \ldots$

3.15 Slip vector in $3 \mathrm{D}$ space . . . . . . . . . . . . . . 66

4.1 Calculating plane indices . . . . . . . . . . . . . . . 68

4.2 Evolution of the dislocation $(a) \ldots \ldots \ldots 6$

4.3 Evolution of the dislocation $(b) \ldots \ldots \ldots$. . . . . . . 70

4.4 Evolution of the dislocation $(c) \ldots \ldots \ldots 71$

4.5 Evolution of the dislocation $(d) \ldots \ldots \ldots 72$

4.6 Contour of the slip vector for $\mathrm{Cu}(001) \ldots \ldots \ldots 74$

4.7 Vector graph of slip vector for $\mathrm{Cu}(001) \ldots \ldots \ldots \ldots$

4.8 Front view of $\mathrm{Cu}(001) \ldots \ldots \ldots \ldots$

4.9 Prediction curves of $\mathrm{Cu}(001) \ldots \ldots \ldots \ldots$

4.10 Predicted and actual results for $\mathrm{Cu}(001) \ldots \ldots . \ldots . . \ldots 8$

4.11 Simultaneous comparison for $\mathrm{Cu}(110) \ldots \ldots . \ldots . \ldots 5$

vii 
5.1 Prediction curves for $\mathrm{Ni}(001) \ldots \ldots \ldots . \ldots . \ldots . \ldots 9$

5.2 Predicted and actual results for $\mathrm{Ni}(001) \ldots \ldots . \ldots . . \ldots . . \ldots 92$

5.3 Nucleation for $\mathrm{Cu}(110) \ldots \ldots \ldots . \ldots . \ldots . \ldots . \ldots 9$

5.4 Slip vector contour for $\mathrm{Cu}(110) \ldots \ldots \ldots$. . . . . . . . . . 95

5.5 Vector graph of $\operatorname{slip}$ vector for $\mathrm{Cu}(110) \ldots \ldots$. . . . . . . . 96

5.6 Front view of $\mathrm{Cu}(110) \ldots \ldots \ldots$. . . . . . . . . . 97

5.7 Prediction curves for $\mathrm{Cu}(110) \ldots \ldots \ldots$. . . . . . . . . . 98

5.8 Predicted and actual results for $\mathrm{Cu}(110) \ldots \ldots . \ldots 9$

5.9 Prediction curves for $\mathrm{Ni}(110) \ldots \ldots \ldots$. . . . . . . . . . 103

5.10 Predicted and actual results for $\mathrm{Ni}(110) \ldots \ldots$. . . . . . . 104

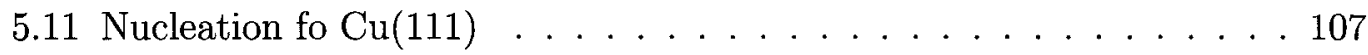

5.12 Slip vector contour for $\mathrm{Cu}(111) \ldots \ldots . \ldots 108$

5.13 Vector graph of slip vector for $\mathrm{Cu}(111) \ldots \ldots . . . \ldots 109$

5.14 Front view of $\mathrm{Cu}(111) \ldots \ldots . \ldots . . \ldots 110$

5.15 Prediction curves for $\mathrm{Cu}(111) \ldots \ldots . \ldots . \ldots 111$

5.16 Predicted and actual results for $\mathrm{Cu}(111) \ldots \ldots . \ldots 113$

5.17 Prediction curves for $\mathrm{Ni}(111) \ldots \ldots \ldots 116$

5.18 Predicted and actual results for $\mathrm{Ni}(111) \ldots \ldots . \ldots 117$

5.19 3D rotation of coordinate system $\ldots \ldots \ldots . \ldots \ldots$

viii 


\section{List of Tables}

4.1 Prediction table for $\mathrm{Cu}(001) \ldots \ldots \ldots$. . . . . . . . . . 82

4.2 Table of simulation results . . . . . . . . . . . . 83

5.1 Prediction table for $\mathrm{Ni}(001) \ldots \ldots . \ldots . \ldots 90$

5.2 Prediction table for $\mathrm{Cu}(110) \ldots \ldots \ldots 10 . \ldots \ldots$

5.3 Prediction table for $\mathrm{Ni}(110) \ldots \ldots \ldots$. . . . . . . . . . 105

5.4 Prediction table for $\mathrm{Cu}(111) \ldots \ldots \ldots$. . . . . . . . . 114

5.5 Prediction table for $\mathrm{Ni}(111) \ldots \ldots \ldots \ldots$ 


\section{Nomenclature}

\begin{tabular}{|c|c|}
\hline $\mathrm{A}_{i}$ & Contact area at atom site $i$ \\
\hline$a$ & Contact radius \\
\hline$a_{0}$ & Original lattice constant for a specific fcc crystal \\
\hline $\mathrm{BCC}$ & Body centered cubic \\
\hline$\vec{b}$ & Burgers vector \\
\hline$C$ & Effective stiffness of the indenter \\
\hline CADD & Coupled atomistics and discrete dislocation \\
\hline EAM & Embedded atom method \\
\hline $\mathrm{E}_{I}$ & Sum of potential between the indenter and its neighbor atoms \\
\hline $\mathrm{E}_{i}$ & Site energy of atom $i$ \\
\hline $\mathrm{E}^{*}$ & Reduced or combined tip/substrate modulus \\
\hline $\mathrm{E}_{t i p}$ & Tip elastic modulus \\
\hline $\mathrm{E}_{s u b}$ & Substrate elastic modulus \\
\hline$\Delta \mathrm{E}$ & The change of energy \\
\hline FEM & Finite Element Method \\
\hline FCC & Face centered cubic \\
\hline$F_{i}\left(\rho_{h, i}\right)$ & Energy to embed atom $i$ into the background with $\rho_{h, i}$ \\
\hline$\vec{f}_{i}$ & Indenter caused force on atom $i$ \\
\hline $\mathrm{HCP}$ & Hexagonal close packed \\
\hline
\end{tabular}




\begin{tabular}{|c|c|}
\hline$I, J$ & Refer to Cartesian components \\
\hline LOPI & Local optimal point interpolating \\
\hline 1 & Unit line direction \\
\hline $\mathrm{MD}$ & Molecular Dynamics \\
\hline $\mathbf{m}$ & Unit vector in the direction of Burgers vector \\
\hline $\mathrm{N}_{m, l}$ & Stress gradients related value \\
\hline $\mathrm{N}_{\text {crit }}$ & Critical material constant \\
\hline$n$ & Number of nearest neighbors to atom $\alpha$ \\
\hline$n_{s}$ & Number of slipped neighbors \\
\hline $\mathrm{P}$ & Indenter load \\
\hline $\mathrm{PBC}$ & Periodic boundary conditions \\
\hline$p_{0}$ & Maximum pressure on the contact circle \\
\hline QC & Quasi-continuum method \\
\hline$R$ & Radius of the indenter \\
\hline$R_{i j}$ & Distance between atom $i$ and $j$ \\
\hline$r_{c u t}$ & Cut-off radius \\
\hline$r_{i}$ & Radius of an individual atom $i$ \\
\hline$r_{I j}$ & Distance from the center of indenter to the $j$ th atom \\
\hline$s^{\alpha}$ & Slip vector \\
\hline TEM & Transmission electron microscopy \\
\hline $\mathbf{T}$ & Stress tensor \\
\hline curl T & Curl of the stress tensor \\
\hline
\end{tabular}




$\begin{array}{ll}\vec{x}^{\alpha \beta} & \text { Vector differences of atoms } \alpha \text { and } \beta \text { in the current positions } \\ \overrightarrow{\mathrm{X}}^{\alpha \beta} & \text { Vector differences of atoms } \alpha \text { and } \beta \text { in the reference positions } \\ \sigma_{i}^{\mathrm{IJ}} & \text { Atomic level stresses at atom } i \\ \sigma_{i}^{\mathrm{K}} & \text { Indenter caused stress on atom } i \\ \sigma_{r}, \sigma_{\theta} & \text { Stress within the framework of cylinder coordinate system } \\ \sigma_{x}, \sigma_{y} & \text { Stress within the framework of Cartesian coordinate system } \\ \tau & \text { Resolved shear stress } \\ \tau_{c r s s} & \text { A critical resolved shear stress } \\ \varepsilon_{i j}^{I J} & \text { Strain between atoms } i \text { and } j \\ \psi\left(r_{I j}\right) & \text { Repulsive potential between indenter and atom } j \\ \delta & \text { Indenter displacement } \\ \rho_{h, i} & \text { Host electron density at atom } i \\ \phi_{i j}(\text { Rij }) & \text { Core-core pair repulsion between atom } i \text { and } j\end{array}$




\section{Chapter 1}

\section{Introduction}

\subsection{Criterion of Dislocation Nucleation}

A fundamental transition in the mechanical behavior of materials is the shift from elastic (reversible) to plastic (irreversible) deformation. Nucleation and kinetics of defects at the atomic scale provide the most fundamental information about the strength and ductility of materials, which are related to the various deformation mechanisms. A material in which dislocations are easy to move and multiply will normally be soft and malleable, whereas a material in which dislocations can neither move nor be easily created will be hard and brittle. Although the fact has been recognized that the generation of new dislocations (line defects in lattice crystals) is an important aspect of crystal defect physics, a correct understanding of the mechanical conditions which lead to defect nucleation still remaines elusive. As such, there remains a need for nanoscale interpretation and prediction of dislocation nucleation. The aim of this thesis is to examine a stress gradient based nucleation criterion (so called "Acharya/Miller" criterion [1]) and demonstrate the criterion's ability to predict dislocation nucleation with fully three dimensional atomic simulation.

In current work, nanoindentation [2] of fcc single crystal metals (e.g., $\mathrm{Cu}, \mathrm{Ni}$ ) is simulated using the Molecular Dynamics(MD) [3] method. The simulations are subsequently analyzed to determine when and how dislocations nucleate during 
indentation, and then these observations are compared with predictions from the "Acharya/Miller criterion" and the criterion's efficiency is proved for predicting defect nucleation. The current work focus on the incipient plasticity (the very initial stage of plastic activity during nanoindenation) to investigate the mechanisms whereby dislocations are nucleated, and to provide a clear and controllable test. The success of the criterion in these conditions will lend confidence to more general application of the criterion.

\subsubsection{The previous nucleation criteria}

During the past years, the importance of defect nucleation criterion has been recognized to predict the mechanical conditions leading to initial plasticity of crystalline materials. A few nano-indentation simulations [4],[5],[6],[7],[8],[9],[10],[11] similar to current work have commonly revealed the process of homogeneous defect nucleation. Other related MD simulations also reveal the details of extended dislocation configurations and the complex processes of dislocation multiplication $[12],[13],[14]$. Furthermore, the details of heterogeneous defect nucleation and interactions in rela-

tively complex structures of nano-crystalline materials have also been described [15], [16]. At the same time, several criteria of dislocation nucleation based on different continuum and atomistic models have been introduced, and are summarized here.

\section{"Schmid-like" criterion}

The motion of dislocations in fcc crystals is often observed to obey Schmid's Law (the dislocation will move when the resolved shear stress acting on it reaches a critical, material specific value), while other crystal structures, such as bcc crystal, follow the so-called "non-Schmid" dislocation behavior [17] (the motion of dislocation depends on stress components other than the resolved shear stress). A natural extension of Schmid's Law, whereby it has been assumed that a critical, material specific stress level must be reached at a point in the crystal for nucleation to occur, has been 
adopted to give a "Schmid-like" criterion [18]. Simulations demonstrate that this commonly held notion of nucleation criterion based on the magnitude of local stress components is incorrect, and the nucleation location predicted by this criterion does not agree with the real location.

\section{Rice's criterion}

Rice's study of dislocation nucleation from a crack tip leads to a nucleation condition based on the so-called "unstable stacking fault" energy of crystalline slip. Although this criterion serves some other types of nucleation (e.g., as a basis for the studies of dislocation nucleation from a crack [19], nucleation from the corner of a knifeedge indenter [20], and prediction of twinning near a crack tip [21]), it has not been extended to the instances of homogeneous nucleation away from singular points like crack tips or indenter corners.

\section{$" \wedge-$ criterion"}

More recently, an energy-based instability criterion of dislocation nucleation, the so called " $\wedge$-criterion", was developed based on instability analysis and has been examined to correctly predict the location and slip character (slip plane and Burgers vector) of the corresponding dislocation nucleation[11], [10]. This criterion remains a fully continuum method, and its implementation is in a standard finite element software package and requires that the user supply the exact Cauchy-Born constitutive relation.

\subsubsection{Description of the "Acharya/Miller" criterion}

In this thesis, a stress gradient based criterion, named "Acharya/Miller" criterion [1] and developed from the continuum thermomechanical considerations to correctly predict dislocation nucleation, is examined for dislocation nucleation.

The essential standpoint of this criterion is that there is a material property $\mathrm{N}_{c r i t}$, 
when the stress at some atom position satisfies the following condition, the corresponding dislocation will nucleate.

$$
\mathrm{N}_{m, l} \geq \mathrm{N}_{\text {crit }}
$$

where $\mathrm{N}_{m, l}$ depends on the stress gradients, the unit dislocation line $\mathbf{l}$, and the unit Burgers vector $\mathbf{m}$ on the slip plane, which will be described in detail in section 2.4.3. In Acharya and Miller's original work [1], the criterion had already been examined to accurately predict dislocation nucleation in two-dimensional atomistic models. It also provided a critical material constant, $\mathrm{N}_{\text {crit }}$, to correctly predict the location and moment of nucleation. Furthermore, they also demonstrated that the "Schmid-like" criterion is incorrect. To continue their work, current work quantitatively simulates nanoindentation by the fully three-dimensional MD method with different crystal orientations shown in Figure 1.1.

\subsection{Overview of the thesis}

Chapter 1 includes a brief introduction of the previous nucleation criteria, the fundamental ideas of the "Acharya/Miller" criterion and the simulation approaches used in current work.

Chapter 2 will give a summary of similar previous work done in this field and related fields on dislocation activities. Furthermore, an in-depth overview of some dislocation nucleation criteria (e.g., "Schmid-like" criterion, " $\wedge$-criterion", and the "Acharya/Miller" criterion) and a simple description of the computational and experimental approaches used to study the dislocation nucleation will also be provided. Chapter 3 will give a detailed description of the MD simulation approach used in current simulations. It shows how the model is built and the determination of 


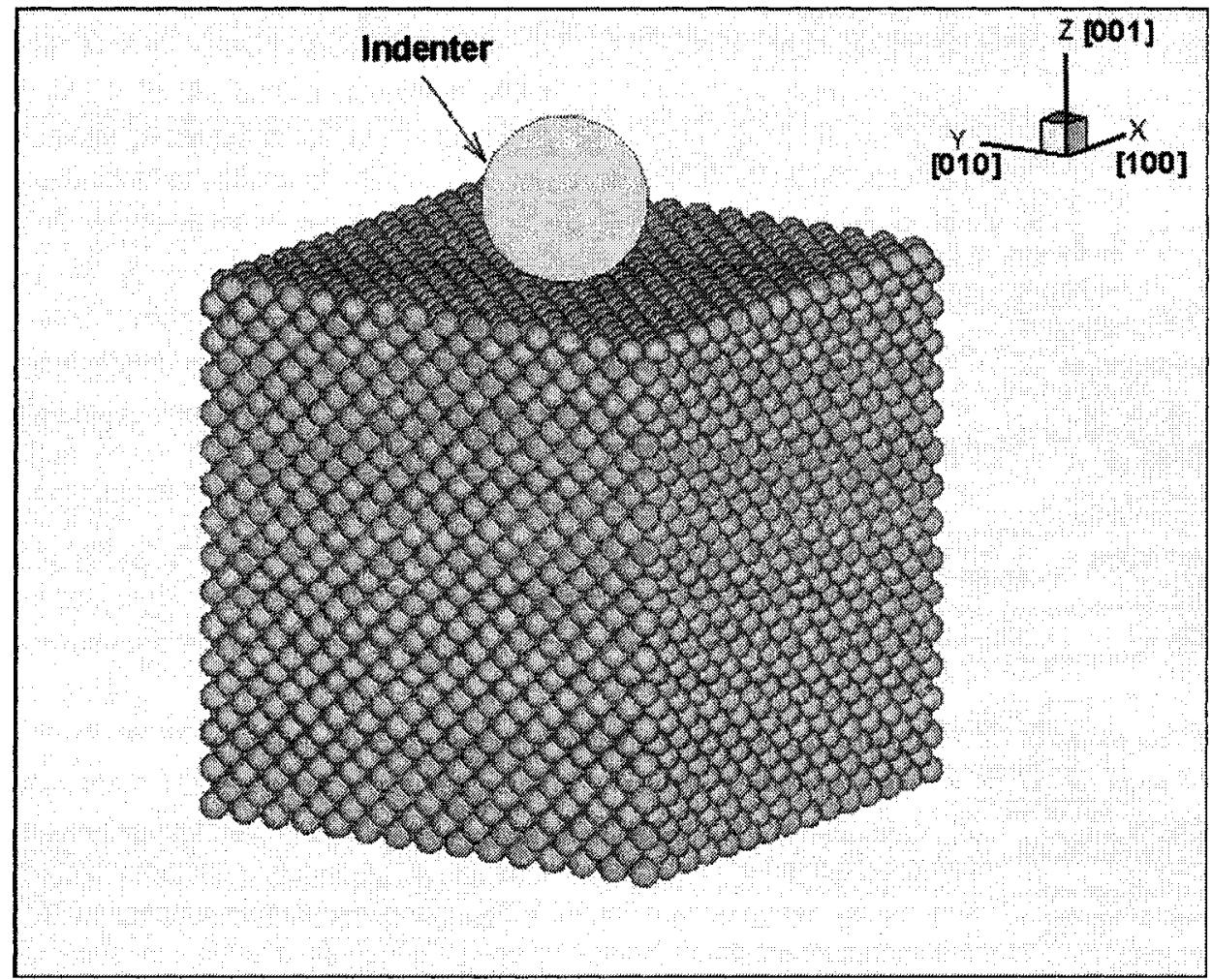

Figure 1.1: Diagram of a nano-indentation simulation example, separately rotate a fcc perfect single crystal structure to make the indentation surface either (001) as shown here, or the (110), (111) plane. 
related parameters, and also gives an explanation of the embedded atom method and periodic boundary condition used in this simulation. Then, an essential part of this thesis, a related procedure is designed to evaluate the current criterion.

In order to test the criterion, two necessary steps will be required. One is a way to find stress and stress gradients in an atomistic simulation, another is a way to identify when a dislocation nucleates and how to determine its character. All these techniques are presented in chapter 3 .

The detailed simulation results and related discussion are described in chapter 4 , and the conclusions are summarized in chapter 5. More results and details of a calculation method for coordinate transformation, which are not shown in the body of the thesis, are given in the appendices. 


\section{Chapter 2}

\section{Background and Literature Review}

Many commonly used metals such as copper, aluminium, nickel and their alloys, have face-centered cubic (fcc) crystal structures. The pure metals are soft and ductile but can be hardened considerably by plastic deformation and alloying. The deformation behavior is closely related to the atomic structures of the core of dislocations [22]. In this thesis, fcc crystals are exclusively focused.

\subsection{The face centered cubic (fcc)}

For most engineering materials including metals and ceramics, the atoms are arranged in a manner that repeats itself periodically in three-dimensional space. This periodically repeating pattern of arrangement is termed as crystalline, and the arrangement of the atoms is called the crystal structure. To visualize the structures, atoms of crystalline materials are approximately regarded as hard spherical balls having well defined diameters and holding together by attractive forces. This is termed as the atomic hard sphere model in which each single spherical ball touches with its neighbor atoms.

The crystalline structures of most pure metals at room temperature belong to one of three relatively simple types, which are body-centered cubic (bcc), face-centered cubic (fcc), and hexagonal close packed (hcp). Typical metals with the fcc structures 
include $\mathrm{Al}, \mathrm{Ni}, \mathrm{Cu}, \mathrm{Ag}$, and $\mathrm{Au}$. Figure 2.1 shows the fcc crystal structure, the atoms are positioned at the corners of the unit cell and at the centers of all the cube faces.

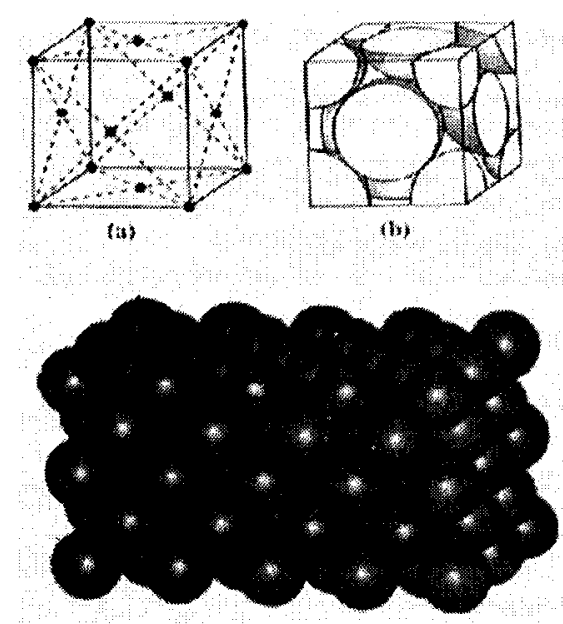

(c)

Figure 2.1: Face-centered cubic (fcc) structures for metals (a) the arrangement of lattice points for a unit cell; (b) the actual packing of atoms within the unit cell; (c) the repeating fcc structures, equivalent to many adjacent unit cells (Taken from [23])

\subsection{1 fcc slip system}

Observing the fcc hard sphere model in Figure 2.1, the atoms touch along the $\langle 110\rangle$ directions which are termed the close-packed directions, the $\{111\}$ planes are the close packed planes and contain three close-packed directions which are $60^{\circ}$ apart. The stacking sequence of the $\{111\}$ planes is the ABCABCABC... arrangement as shown in Figure 2.2. A slip plane and a slip direction in the plane are termed a slip system. The slip plane of crystal structures is normally the close-packed plane and the direction of slip is the close-packed direction. Therefore, the slip of fcc crystals often occurs on $\{111\}$ planes in $\langle 110\rangle$ directions, fcc crystals have four $\{111\}$ planes and three $\langle 110\rangle$ directions, and thus in total have twelve $\{111\}\langle 110\rangle$ slip systems. 


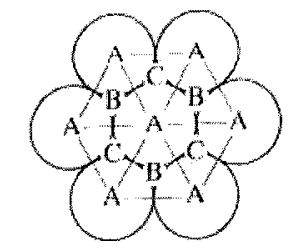

(a) Slacking of (111) plancs

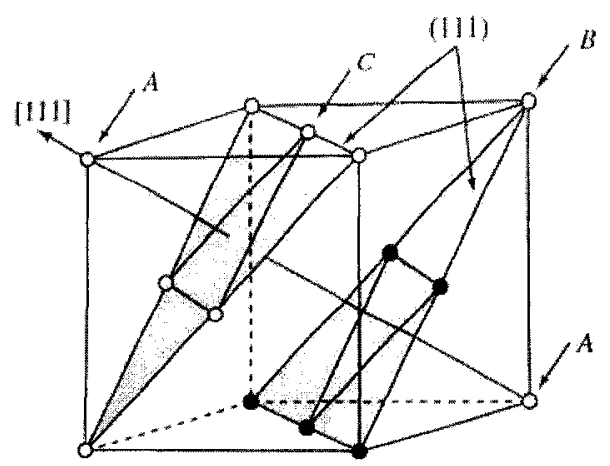

(b) Face-centered cubic

Figure 2.2: The $\{111\}$ close-packed planes of atoms in fcc structures (Taken from [23])

\subsection{Dislocations in fcc crystal structures}

The concept of dislocations, which are linear defects of crystal structures, arose primarily from the study of plastic deformation processes and is used to explain the ductility in crystalline materials. A basic understanding of dislocations can be introduced by the idea of slip, that means the sliding or successive displacement of one plane of atoms over another in a crystal. According to the configuration and formation of dislocation, there exist three type of dislocations (i.e., edge, screw and mixed dislocation.)

\subsubsection{Edge dislocation}

Figure 2.3 shows the illustration of slip, where a unit slip has occurred only over a portion of the crystal, with an unslipped region on the other part of the crystal. The slipped region and the unslipped region are separated by the dislocation. As 
shown in the figure, dislocation line FE and an extra half plane EFGH are formed, although it is emphasized that a uniform slip across the slip plane AEFD does not occur in practice. Considering the atoms in the fcc structure of Figure 2.3 and looking through along line EF, the atom positions of the dislocation are shown in Figure 2.4, and this type of dislocation is called an edge dislocation. Figure 2.5 shows the same idea with a group of dynamically changed atomistic views, where the edge dislocation moved plane by plane and finally produced a unit step of slip under the applied shear stress. Figure 2.6 shows how slip, dislocation and dislocation motion lead to plastic deformation if the dislocations are able to move across the slip plane from one side of the crystal to the other [22].

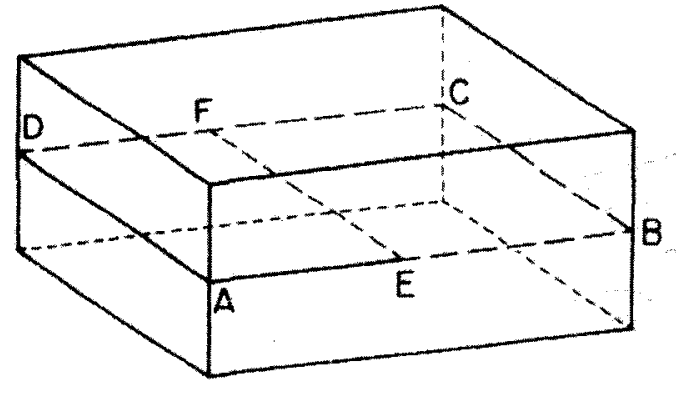

(a)

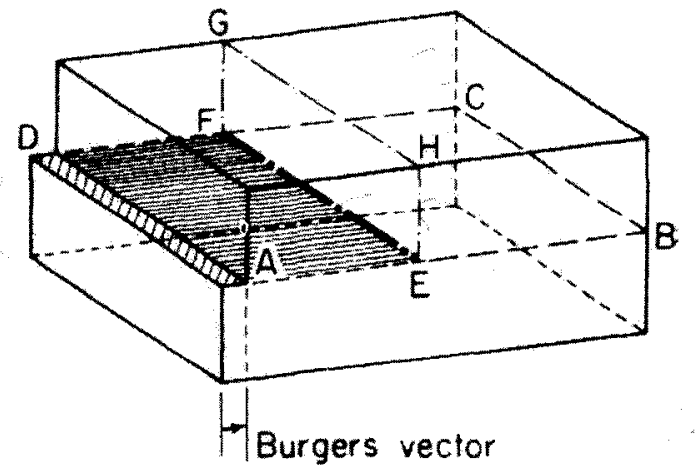

(b)

Figure 2.3: Formation of a pure edge dislocation FE [22] 


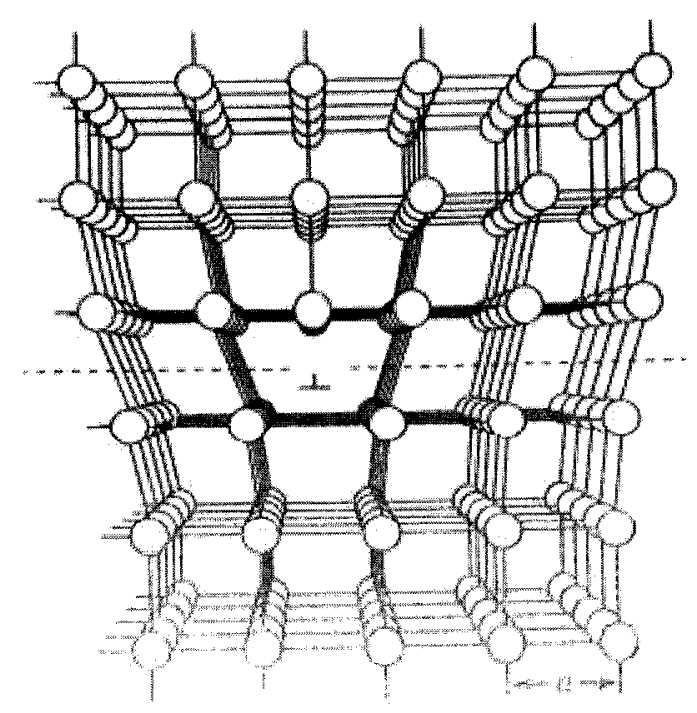

Figure 2.4: Edge dislocation, the linear defect is represented by the edge of an extra half-plane of atoms. (Taken from [23])

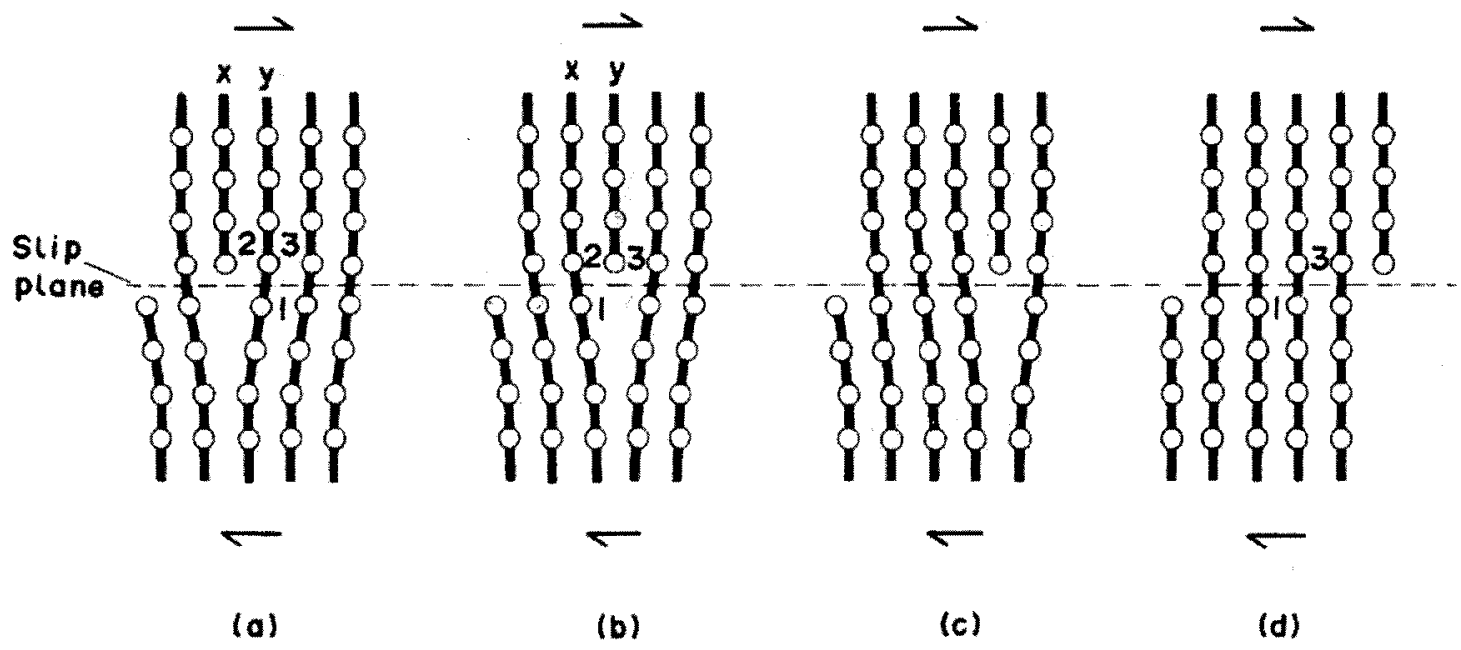

Figure 2.5: Slip of an edge dislocation. (Taken from [22]) 

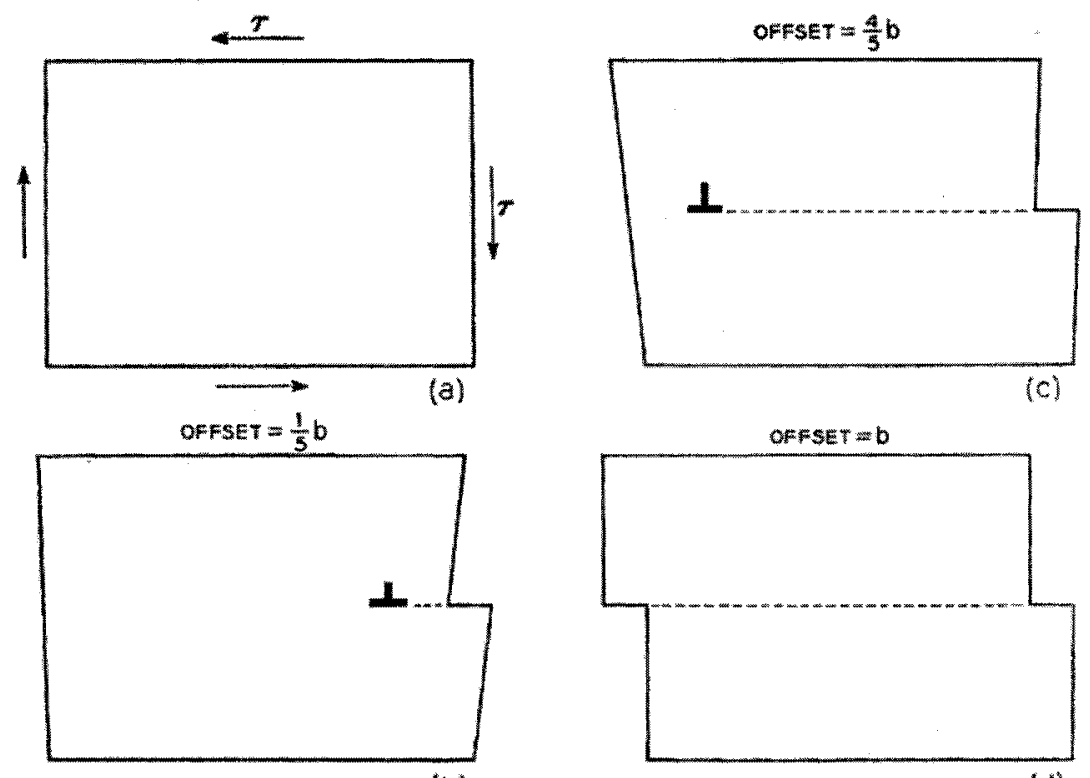

(b)

(d)

Figure 2.6: Dislocation motion leads to plastic deformation. (a) application of shear stress to deform the crystal. (b) and (c) dislocation has moved a fraction of the offset b. (d) dislocation has moved to the other end of the crystal. (Taken from [24])

\subsubsection{Screw dislocation}

A screw dislocation can be formed when a shear stress is applied on a block as to produce the distortion shown in Figure 2.7, the left region of the crystal has been shifted by one atomic distance to the upper relative to the right part.

\subsubsection{Burgers vector}

The most important definition of a dislocation is given in terms of the Burgers circuit, it is any atom to atom path taken in a crystal containing dislocations which forms a closed loop. Such a path is illustrated in Figure 2.8(a) and Figure 2.9(a). However, if the same atom to atom path is made in a dislocation-free crystal, the circuit does not close shown as Figure 2.8(b) and Figure 2.9(b). The vector required to complete the circuit is called the Burgers vector $\vec{b}$, which represents the magnitude 


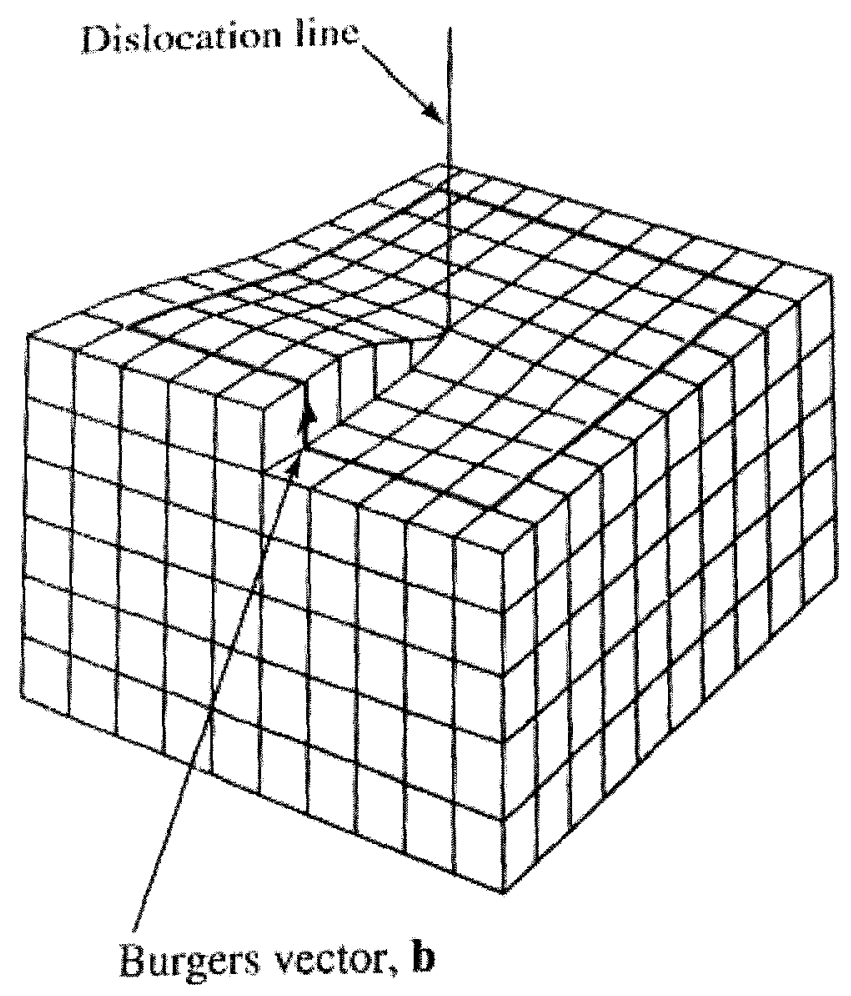

Figure 2.7: Screw dislocation, the spiral stacking of crystal planes leads to the Burgers vector being parallel to the dislocation line (Taken from [23]) 
of the structural defect. It is obvious that edge and screw dislocations are the two kinds of basic geometry of dislocations. The Burgers vector of an edge dislocation is perpendicular to the dislocation line, while the Burgers vector of a screw dislocation is parallel to dislocation line. Sites where the crystal stacks have been disrupted, such as an extra half plane produces to form an edge dislocation; a spiral produces a screw dislocation [22].

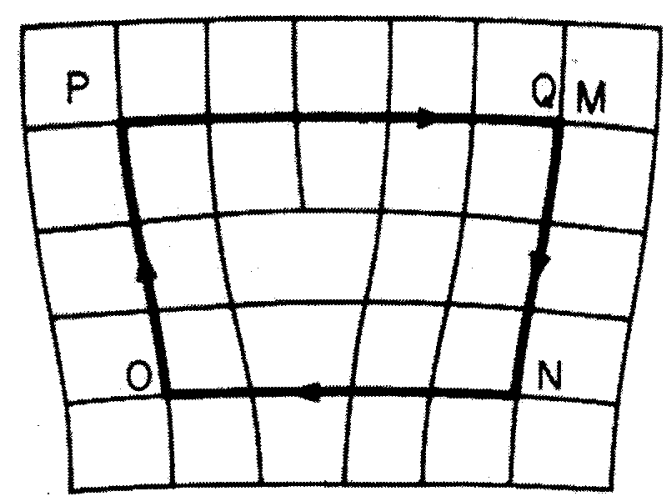

(a)

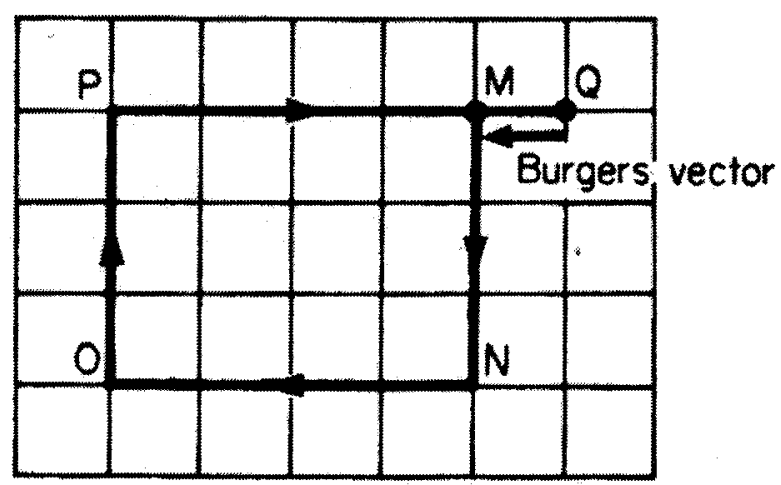

(b)

Figure 2.8: (a) Burgers circuit rounds an edge dislocation; (b) The same circuit in a perfect crystal and the closure failure is the Burger vector (Taken from [22]) 

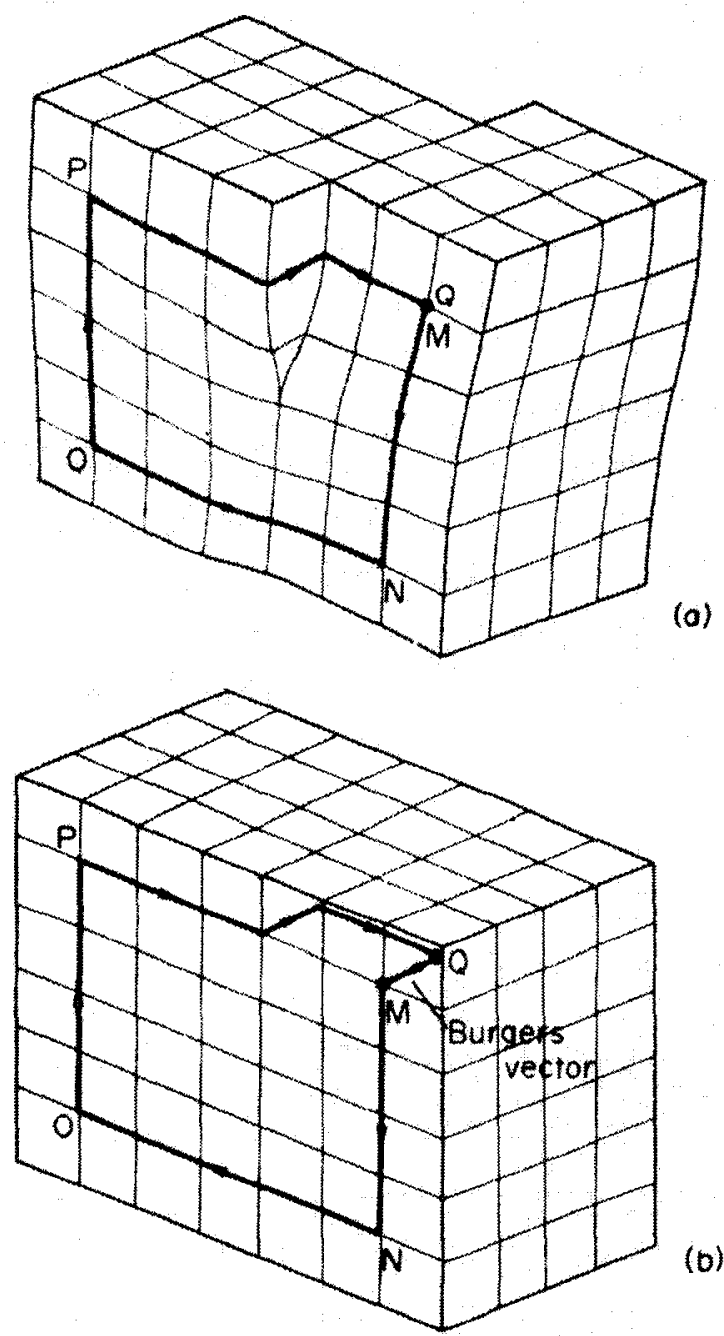

Figure 2.9: (a) Burgers circuit rounds a screw dislocation; (b) The same circuit in a perfect crystal and the closure failure is the Burger vector (Taken from [22]) 


\subsubsection{Mixed dislocation}

Most dislocations found in materials are neither pure edge nor pure screw but a combination of both of them, this condition is termed a mixed dislocation. Normally, the dislocation line lies at an arbitrary angle to its Burgers vector and it has mixed edge and screw character. Figure 2.10 shows the three different types of dislocations for an actual dislocation. The dislocation character changes from screw, mixed and finally edge dislocation. The lattice distortion produced away from the two faces is mixed. It is worthy to emphasis that even though a dislocation changes direction and type (e.g., edge to mixed or screw), the Burgers vector will be the same at all points along the dislocation line.

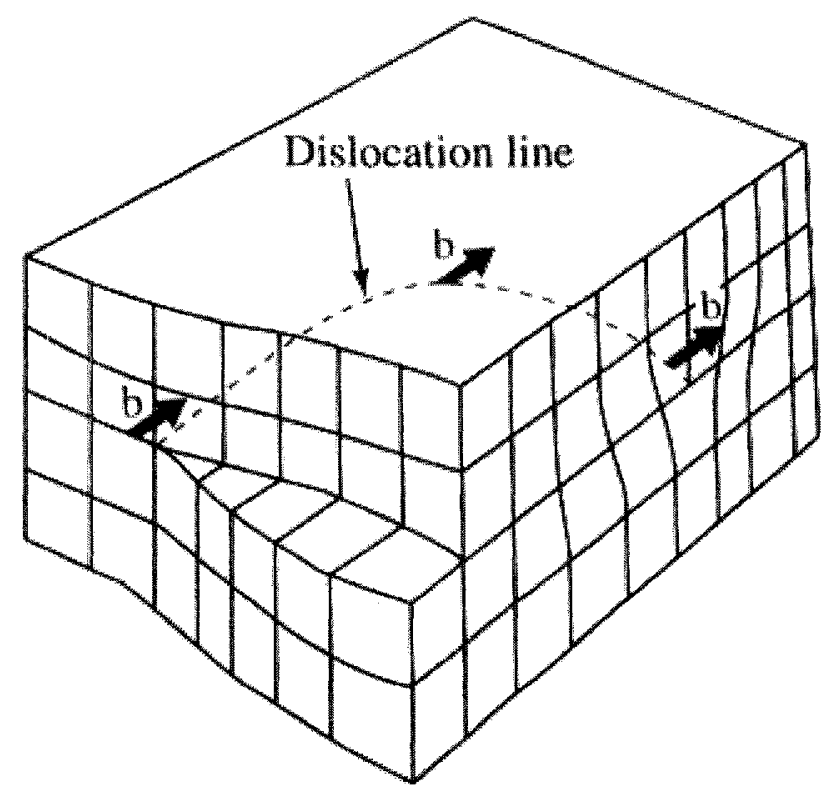

Figure 2.10: Mixed dislocation, its Burgers vector consistents with the pure edge and pure screw regions. (Taken from [23]) 


\subsubsection{Stacking faults \& Shockley partial dislocations}

A stacking fault is a planar defect. As its name implies, it is a local region in the crystal where the regular stacking sequence has been interrupted. When a stacking fault ends inside a crystal, the boundary in the plane of the fault, separating the faulted region from the perfect region of the crystal, is a partial dislocation, named a Shockley partial dislocation. As one of important partial dislocations, the Shockley partial dislocation is associated with slip and has been recognized in fcc metals. The formation of a Shockley partial edge dislocation is illustrated in Figure 2.11 and can be compared with the formation of an edge dislocation in an elastic model shown in Figure 2.4. The diagram represents a $(10 \overline{1})$ section through the lattice. The close-packed (111) plane is normal to the plane of the diagram. The $A$ layers rest on $C$ layers at the right of the diagram and the lattice is perfect, however, the $A$ layers along $L M$ have slipped in a [1리] direction to a $B$ layer position to produce a stacking fault and partial dislocation at the left of the diagram [22].

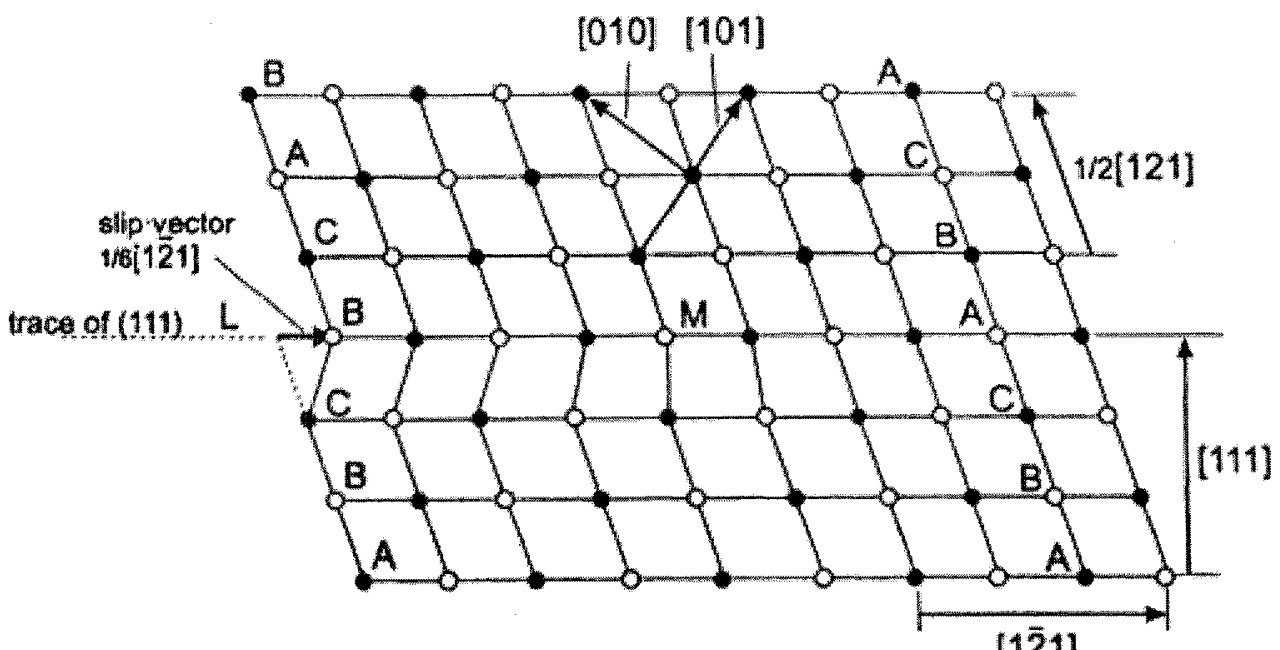

[1리]

Figure 2.11: Formation of an $\frac{1}{6}[1 \overline{2} 1]$ Shockley partial dislocation at $M$ due to slip along $L M$. (Taken from [22]) 
For fcc crystal structures, on any of the four $\{111\}$ slip planes there are three $\langle 110\rangle$ directions and three $\langle 112\rangle$ directions, as shown for the (111) plane in Figure 2.12. A perfect dislocation will split into two partial dislocations, for example, on the (111) plane, the Burgers vector reactions are given by:

$$
\begin{aligned}
& \frac{1}{2}[\overline{1} 10] \rightarrow \frac{1}{6}[\overline{2} 11]+\frac{1}{6}[\overline{1} 2 \overline{1}] \\
& \frac{1}{2}[\overline{1} 01] \rightarrow \frac{1}{6}[\overline{2} 11]+\frac{1}{6}[\overline{1} \overline{1} 2] \\
& \frac{1}{2}[0 \overline{1} 1] \rightarrow \frac{1}{6}[1 \overline{2} 1]+\frac{1}{6}[\overline{1} \overline{1} 2]
\end{aligned}
$$

It is necessary in dislocation reactions to ensure that the total Burgers vector is unchanged. The right-hand side of the above reactions formula, for example, is $\frac{1}{6}[\overline{2}+\overline{1}, 1+2,1+\overline{1}]$, which equals the left-hand side. The same result is demonstrated diagrammatically by the vector triangles in Figure 2.12. Since the energy of dislocation is proportional to the magnitude of Burgers vector square [22], the splitting reactions (i.e., from Equation 2.1 to Equation 2.3) can be proved to be energetically favorable by the fact that the value $\frac{a^{2}}{2}$ from Equation 2.5 is greater than the sum of two Shockley value $\frac{a^{2}}{3}$ from Equation 2.4.

A Shockley partial dislocation:

$$
\left(\mathbf{b}=\frac{1}{6}\langle 112\rangle\right): b^{2}=\frac{a^{2}}{36}\left(1^{2}+1^{2}+2^{2}\right)=\frac{a^{2}}{6}
$$

Unit edge dislocation:

$$
\left(\mathbf{b}=\frac{1}{2}\langle 110\rangle\right): b^{2}=\frac{a^{2}}{4}\left(1^{2}+1^{2}+0\right)=\frac{a^{2}}{2}
$$

Shown as Figure 2.13, the unit edge dislocation $\mathbf{b}_{c}$ will split into two Shockley partial dislocations $\mathbf{b}_{p b}$ and $\mathbf{b}_{p a}$, there will be a ribbon of stacking fault between them, and the configuration is called an extended dislocation. Figure 2.14 shows a set of extended dislocations lying on the same slip plane. The stacking fault between the two partial dislocations appears as a parallel fringe pattern. 


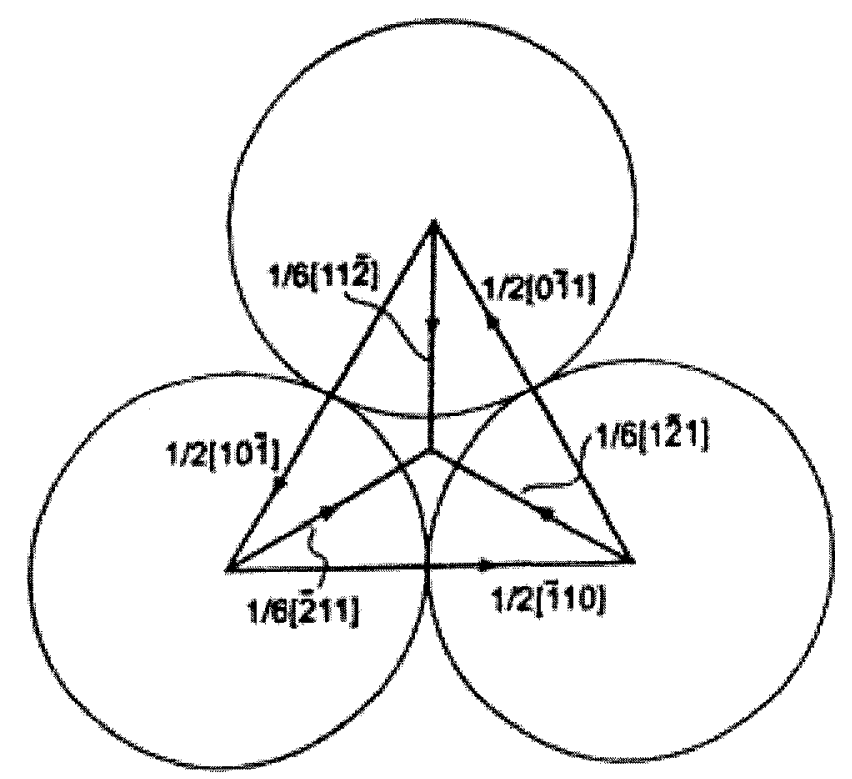

Figure 2.12: Burgers vectors of perfect Shockley partial dislocations in the (111) plane. (Taken from [22])

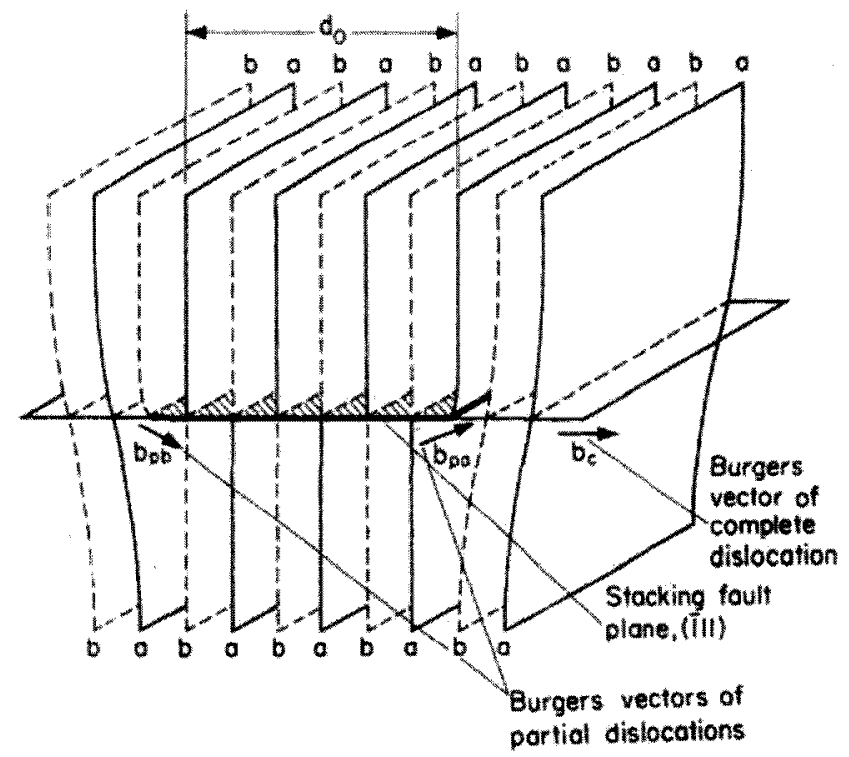

Figure 2.13: Formation of an extended dislocation by dissociation of a unit edge dislocation into two Shockley partial dislocations. (Taken from [22]) 


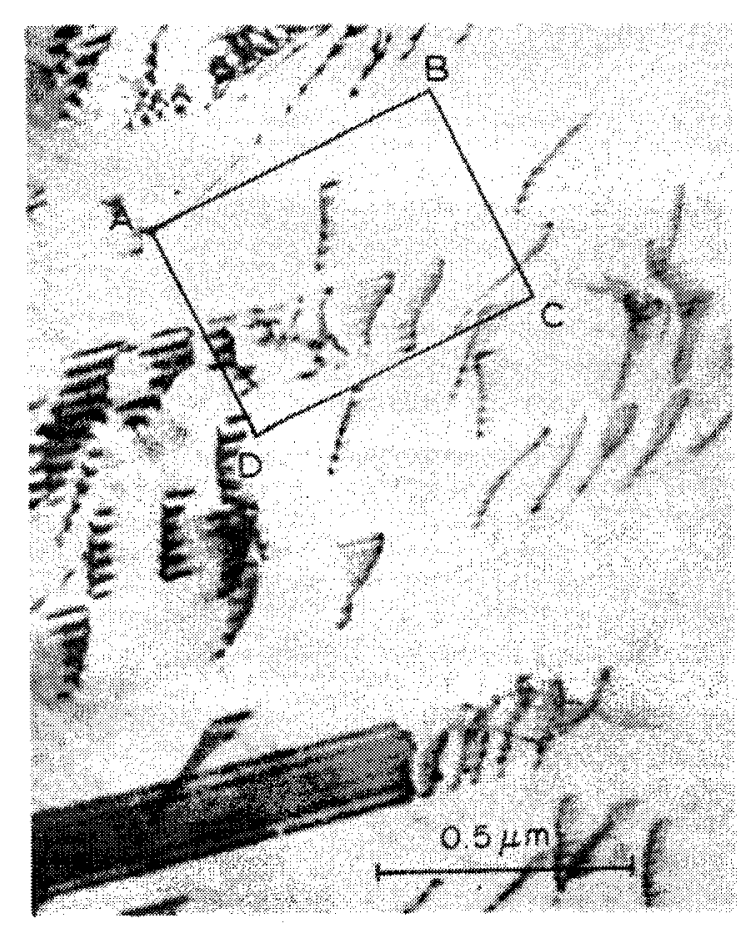

(o)

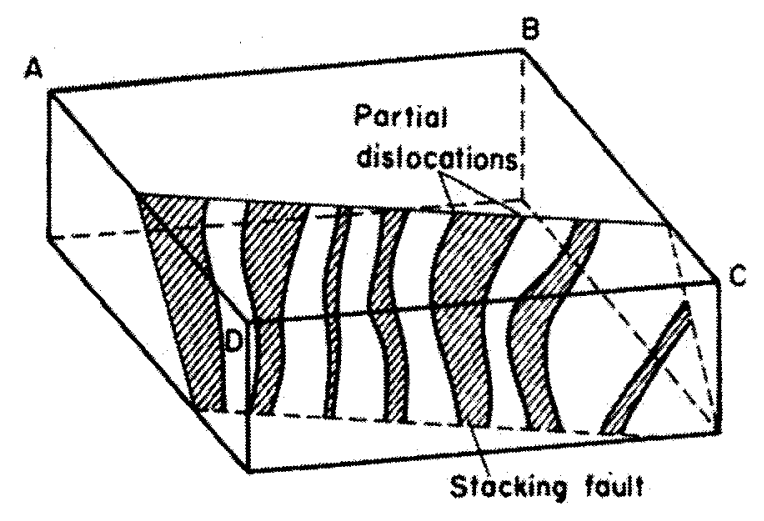

(b)

Figure 2.14: (a) Transmission electron micrograph of extended dislocations in copper. (b) Arrangement of dislocations in the inset in (a) (Taken from [22]) 


\subsection{Previous studies of Dislocation nucleation}

A series of sophisticated computational and experimental tools have been used to explore mechanical response of materials near the atomic level. During the recent years, nanoscale contact experiments $[25],[12],[4],[13],[5],[14],[26],[10],[27]$ and simulations [7],[8],[28],[1],[11] have demonstrated the potential to probe the incipient plasticity and dislocation nucleation in crystals.

\subsubsection{Nanoindentation method}

The verifying of nucleation criteria and investigations of the mechanical properties of surfaces and thin films at atomic scale are done favourably by nanoindentation tests, which means the penetration of the surface to nanometer depths by the molecular dynamics (MD) simulation method or using an indentation device. As the resolution of experimental measurement improves and computational power increases, the worlds of simulation and experiment are able to be bridged.

Nanoindentation has now become a standard technique for evaluating the mechanical properties of thin films. In this procedure, an indenter with dimensions of the order of hundreds of nanometers is pressed into the surface of a solid sample. The nanoindentation test is basically an extension of traditional indentation tests to a very small scale. The traditional tests offer a reasonably unambiguous measure of mean pressure beneath the indenter for a given load which can then be related to the yield strength of the material. However, this relative clarity is lost in nanoindentation. At a very small scale and load, the deformation is characterized by discrete dislocation nucleation events and the subsequent interaction of the small numbers of dislocations that have been generated. This is not the large-scale plasticity observed at the macroscopic scale.

In recent years, the nanoindentation experiments and simulations have demonstrated 
the potential to probe the incipient plasticity or the onset of permanent deformation in crystals. Such studies not only measure the mechanical properties of thin films or material parameters (e.g., elastic constants, yield stress and hardness), but also point out the need for an understanding of the mechanism governing the defect nucleation.

The interpretation of nanoindentation results is complex because of the large number of dependent variables that affect the loading behavior, such as the nature of the surface, the size and geometry of the indenter, the penetration depth, the orientation relative to the specimen crystallography, the elastic anisotropy, the thickness of the substrate, the grain boundary, and pre-existing defect populations [29]. In order to clearly analyze and demonstrate the mechanism of nucleation, in current simulation model, a spherical indenter is used and it is assumed that there is a frictionless contact between the indenter and crystal atoms. Although the simulation result depends on the size of indenter, the evolutions of dislocation nucleation with different indenter radii are very similar for a specific crystal orientation.

\subsubsection{Computational approach}

In recent years, several researchers have elucidated the transitions from the elasticity to the early stage of plasticity during nanoindentation by modeling $3 \mathrm{D}$ fcc crystal structures and observing dislocation activities [4],[13],[7],[14],[15] in metals. However, most observations have not been sufficiently culminated in predictive capabilities.

\section{Atomic scale simulations}

Reviewing the development of nanoindentation simulations, a few famous methods have been used to investigate the mechanical mechanisms of the transition from elastic to plastic deformation. At the early stage of such work, finite element methods 
(FEM) were used in the analysis of indentation problems to provide insight into the true areas of contact and the extent of plasticity. However, FEM requires that the indented body acts as a continuous medium, and thus can not be used to address the discrete nature of incipient plasticity [28]. More recently, the quasi-continuum (QC) method offers an advantage over purely atomistic calculations with regard to system size [28]. The QC methodology is a mixed FEM continuum and MD atomistic method developed to study problems in mechanics of materials where multiple scales are applied simultaneously. The basic idea is that most of the lattice experiences a slowly varying deformation on the atomic scale which is well characterized by the continuum approximation. Atoms are individually represented only in the vicinity of defects or in the presence of mechanical manipulations on the order of the lattice spacing where discrete atomic effects generally become important, whereas the rest of the system is divided into multiple atom elements that are each characterized by a representative atom from that element [29]. However, the 2D nature of the QC method is an idealization of the problem, which limits the comparisons between calculations and real experiments. Ultimately, a fully 3D MD method of nanoindentation, which is applied for a single crystal fcc structure, has been used to show the process of dislocation nucleation without considering grain boundaries and distributions of defects [28].

Comparing with other simulation methods (e.g., FEM and QC method), the fully 3D MD method has been shown its capability of preserving accurate atomistic details of dislocation nucleation for a single crystal structure and explicating the atomic level mechanism of dislocation motion. Although MD simulation is limited by the achievable length and time scales, the approach has the distinct advantage of revealing the emerging dislocation. The simulation results show that the direct MD simulations conducted for a small system can provide the required visualization and precise control over a very well defined time period. 


\section{MD principle [3]}

Among various methodologies of atomic level simulation, the MD method is one of the most popular tools to investigate mechanical behaviors of materials. It can provide the atomic details in this analysis, while the continuum simulations can not. There are two basic assumptions made in standard MD simulations: one is that no mass changes in the system or the number of atoms in the system remains the same; the other is that molecules or atoms are described as a number of interacting material points, whose motion is described dynamically with a vector of instantaneous position and velocity. For example, a single crystal fcc structure is built by representing each atom in the lattice space with its three dimensional coordinates and placing them in a simulation box. For each atom, the relationship between its coordinates and its associating interatomic potentials is represented by the Newton's equations of motion, and its movement is computed explicitly at each time step during the simulation. For this purpose, time integration algorithms have been devised to solve the equations of motion based upon truncated Taylor's expansions with respect to time.

MD simulations have become a powerful tool for elucidating complex mechanics phenomena demonstrated by many researchers. However, for the study of problems such as nanoscale coating, ion-beam deposition and nanoindentation, models need to be on the scale of several microns, consisting of billions of atoms, which is too large for current MD simulations. Therefore, a parallelized MD computation has been created to eliminate the computational limitations of serial computer [30].

The MD method concerns the solution of Newton's equation of motion and the equation can be written in the Newton form:

$$
m_{i} \ddot{r}_{i}=-\frac{\partial u\left(r_{1}, r_{2} \cdots r_{n}\right)}{\partial r_{i}} \equiv F_{i}, \quad i=1,2 \cdots N
$$


Here, $F_{i}$ refers to the internal force. $r_{i}$ indicates the instantaneous position and $m_{i}$ is the mass of particle $i . u\left(r_{1}, r_{2} \cdots r_{n}\right)$ is the energy of particle $i$.

\section{Issue of time step in MD [30]}

In MD simulations, the time step is a crucial parameter that determines the computational expense and accuracy. Two criteria must be followed in choosing the value of the time step. Firstly, the time step should be small enough so that the trajectory of each atom is realistic. The truncation error in a simulation is proportional to the power of the time step, thus a large time step would lead to divergent physical behaviors. Secondly, the time step should be large enough so that the simulation is efficient. A constant time step is commonly implanted in MD simulations for the simplicity of the algorithm, and a varying time step can be used in simulating large time-scale phenomena to improve the efficiency of the simulation.

\section{Atomic potentials: Embedded atom method [31]}

A detailed understanding of the energy and structure of defects in metals is required to explain the problems in solid-state physics and material science. Historically, one complementary theory, named the pair potential [32], was proposed to present the reasonable interpretation of the experimental investigation in these areas. However, this theory is frequently restricted by its inherent limitations. The pair potential may be derived by fitting to a set of experimentally known values, the ambiguity of the atomic volume calculation will influence the accuracy of the pair potential calculation when the local environment is different from the uniform bulk. These problems include surfaces, grain boundaries, internal voids.

More recently, an alternative to the pair potential approach, named the embeddedatom method (EAM), has been developed by Daw and Baskes [32]. This is the same 
method used in the works by Kelchner [7] and Zimmerman [15]. The EAM has some prominent advantages comparing with pair potential models, such as, the efficient calculation of energy for arbitrary arrangements of atoms and successfully avoiding the ambiguity of the volume dependence existing in a pair potential model. Because the EAM provides a realistic description of the metallic cohesion, it appears to be a desirable alternative to the pair potential model and is widely used in the energy calculation for MD simulations.

In the EAM, each atom in a solid can be regarded as an impurity embedded in a host comprising all the other atoms. Therefore, the EAM is based upon the recognition that the cohesive energy of a metal is governed not only by the pairwise potential of the nearest neighbor atoms, but also by the embedding energy related to the "electron sea" in which the atom is embedded. The energy of embedding an impurity is a function of the electron density of the unperturbed host (without the impurity), the total electron density in a metal is reasonably approximated by the linear superposition of contributions from the individual atoms. Since this density is well defined at surface, there are no ambiguities involved in this approach.

In order to facilitate the total energy calculation, an additional assumption is made that the total energy of the collection of atoms can be decomposed into a sum of well-defined site energies associating with each atom.

$$
\mathrm{E}=\sum_{i=1}^{N} \mathrm{E}_{i}
$$

where, $\mathrm{E}_{i}$ is the site energy of atom $i$.

According to the EAM, the total energy of the system shown in Equation 2.8 [31] is the sum of embedding energy determined by the electron density and the electrostatic pair energies set by atomic position.

$$
\mathrm{E}_{i}=\frac{1}{2} \sum_{j(\neq i)} V\left(r_{i j}\right)+F_{i}(\bar{\rho})
$$


where $V$ is a pair potential, $r_{i j}$ is the distance between the atom $i$ and $j, F$ is the embedding energy, $\bar{\rho}$ is the electron density at the site of atom $i$ and given by:

$$
\bar{\rho}=\sum_{j(\neq i)} \rho\left(r_{i j}\right)
$$

where $\rho\left(r_{i j}\right)$ is the spherically symmetric electron density around an isolated nucleus. The sum in these equations runs over all atoms which are in the neighborhood of atom $i$, within some cut-off radius $r_{c u t}$. With this approximation for the electron density, the actual computation using this method do not require significantly more work than the use of pair potential models.

Then, the force on atom $k$ is given by:

$$
\bar{f}_{k}=-\frac{\partial \mathrm{E}}{\partial \bar{x}_{k}}=\frac{-\partial}{\partial \bar{x}_{k}}\left(\sum_{i} \mathrm{E}_{i}\right)
$$

\section{Effect of periodic boundary condition [30]}

Due to the computational limitations of serial computer, MD simulations consider only small representative volume of the full system under analysis, the actual effects of the surrounding media are typically ignored with the use of rigid or periodic boundary conditions. Therefore, the physical behaviors and properties of representative volume can be unambiguously attributed to a corresponding macro-scale system of interest. In a small MD system, a large fraction of atoms are located on the surface of the domain and these surface effects dominate the properties of the simulated material. The use of periodic boundary conditions (PBC) can overcome the surface effects to a certain extent.

In general, the PBC may introduce an artificial periodicity into the simulated system, and the effects of PBC depend on the range of the interatomic potentials and the phenomena under investigation. A simulation is considered to be valid only when 
the size of the simulated domain is larger than the cut-off radius of the interatomic potential and the effective range of the phenomena. In current work, since the $\mathrm{Cu}-\mathrm{Cu}$ or $\mathrm{Ni}-\mathrm{Ni}$ interaction is short-ranged (a few $\AA$ ), a valid MD simulation for this case can be based on a simulation domain with the size on the order of tens of nanometers. The use of the fixed or the PBC may not be capable of capturing long-range physical processes.

In MD nanoindentation simulations, rigid boundary conditions are typically applied on the bottom surface of the cubic single crystal with PBC on the side surfaces of the crystal. These boundary conditions may artificially stiffen the materials and the evolution of any emitted dislocations may also be affected by these boundary conditions. Reliability studies of the simulation results require the estimation of PBC effects, and a standard approach is to test the simulation results repeatedly with increasing simulation domain size.

\subsubsection{Experimental approach}

Initial plasticity in nanocrystalline metals (e.g., $\mathrm{Cu}$ and $\mathrm{Ni}$ ) is mainly determined by dislocation nucleation events. Nevertheless, experimental measurements aiming of identifying the mechanism of intrinsic plastic deformation at the atomic level are suffering from some difficulties. For example, synthesis of the "ideal" nanocrystalline bulk samples for conventional mechanical testing (e.g., tensile and compression tests) is still a challenge. Especially, stable and pure nanocrystalline metal samples with grain size on the order of $10 \mathrm{~nm}$ are extremely difficult to prepare due to their poor thermal stability with high purity at room temperature.

Recent development of nanoindentation offers an effective experimental technique to investigate the mechanical properties and the onset of plasticity of materials at the atomic level. For example, samples used for nanoindentations tests can be very small 
in dimension, and synthesizing suitable nanocrystalline samples [25],[12],[4],[13],[7],[14] for nanoindentation tests is much easier than before. Moreover, the onset of plasticity in crystals is demonstrated by discrete bursts in the experimental load-displacement curves, which are attributed to the initial nucleation of individual dislocations.

The displacement controlled nanoindentations are done by pushing the indenter against the sample to a selected penetration depth. Concurrently, mechanical measurements are performed to investigate how the dislocations originate and slip away from the indentation point [4],[27]. Recently, experimental results of dislocation nucleation measurements show a good agreement with the theory of dislocations, the pop-in drop of indenter load for displacement controlled experiment has been observed in metals (e.g., $\mathrm{Al}, \mathrm{Cu}, \mathrm{Ni}$ ) and some semiconductors [5]. The corresponding dislocations were proved by means of microscopy imaging techniques (transmission electron microscopy (TEM), cathodo-luminescence imaging (CL) ). Figure 2.15 shows a CL imaging of experiment results and presents that the Pop-in-effect does not appear for the force value of $0.05 \mathrm{mN}$ (i.e. loading and unloading curves are reversible, hence pure elastic), but the dark point contrast characterising the nucleated dislocations are observed for indentation forces from $0.1 \mathrm{mN}$ onward.

\subsection{Criteria of dislocation nucleation}

Dislocation activities play an important role in the mechanical behavior of materials; Especially, initial plasticity is decided by dislocation nucleation [30]. The displacement-controlled MD simulation with nanoindentation is used to analyze the mechanical conditions which lead to dislocation nucleation in single crystal metals, and the load-displacement response of such simulation provides a direct measure of the effects of small-scale microstructures on strength [33].

In recent years, a number of MD simulations [7],[28],[8],[1],[11] of nanoindentation 


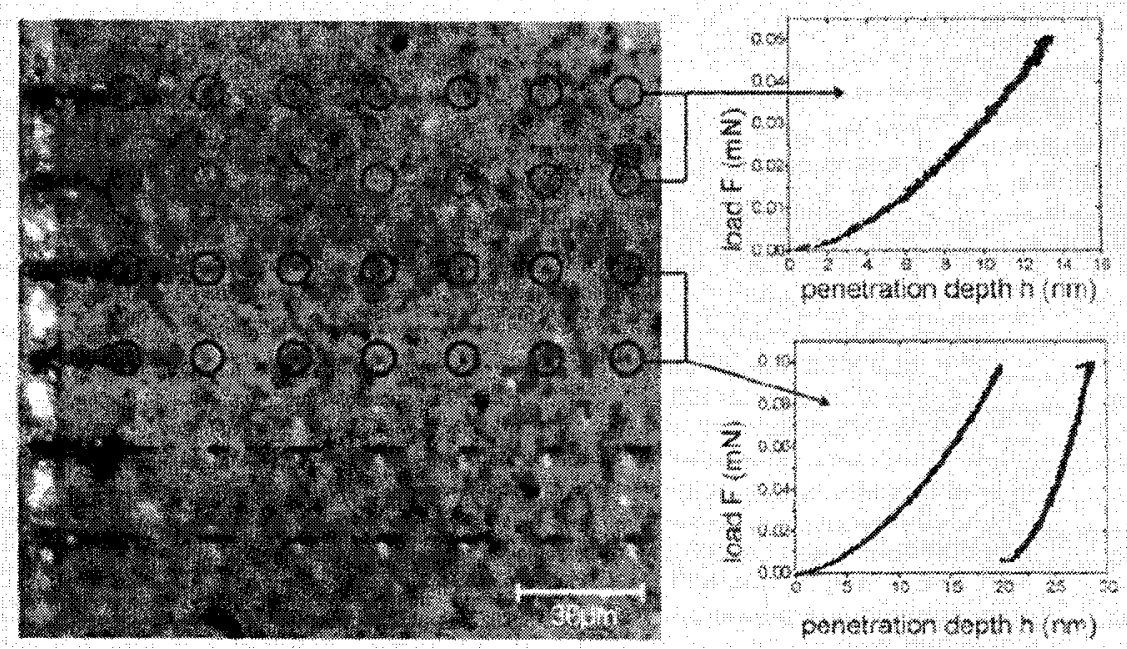

Figure 2.15: Cathodo-luminescence imaging of dislocation loops in ZnSe-layer after indentation with different loads. (Taken from [5])

have led to a great insight into the micro-mechanics of materials. The simulation results demonstrate that the event of dislocation nucleation directly determines the onset of plastic behavior of materials. Therefore, predicting dislocation nucleation events using some efficient criteria becomes the task of nanoscale studies. A few criteria (e.g., the "Schmid-like" criterion and " $\wedge$-criterion") have been provided to elucidate the mechanical conditions at the moment of nucleation.

\subsection{1 "Schmid-like" criterion}

Figure 2.16 shows that the resolved shear stress $\tau$, which lies on the slip plane along the slip direction, is a part of the applied stress. The Schmid's law for dislocation motion states that a dislocation will move when:

$$
\begin{aligned}
& \tau \geq \tau_{\text {crss }} \\
& \tau=\mathbf{s} \cdot \mathbf{T} \cdot \mathbf{n}
\end{aligned}
$$

Or, for uniaxial tension shown in Figure 2.16:

$$
\tau=\frac{F}{A} \cos \phi \cos \lambda
$$


where $\mathbf{s}$ is the unit vector of the slip direction, $\mathbf{T}$ is the stress tensor and $\mathbf{n}$ is the unit vector of the slip plane normal.

A Schmid-like nucleation criterion is extending this idea for dislocation nucleation, and assumes that a critical resolved shear stress, $\tau_{\text {crss }}$, exists for defect nucleation. The criterion is widely used by many researchers to predict nucleation of a specific dislocation at the atom point in crystal structures where $\tau$ first reaches to $\tau_{\text {crss }}$. However, such a criterion has been shown to be a poor predictor of dislocation nucleation [22],[1].

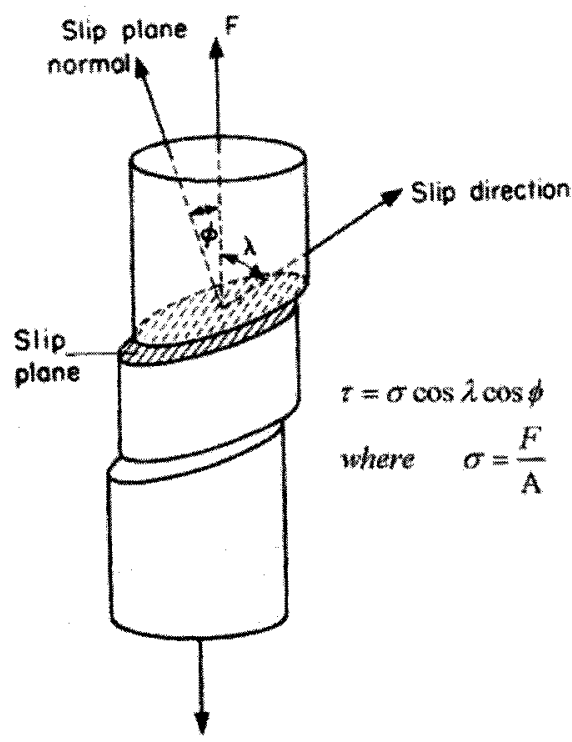

Figure 2.16: Illustration of the geometry of slip in crystalline materials and definition of the resolved shear stress, $\tau$.

\subsection{2 $" \wedge-"$ criterion}

The "^-criterion" [6],[9] was originally developed from an analytical formulation of the elastic limit that predicts the homogeneously nucleated defect, and then it was extended to be a local elastic stability criterion at atomic scale level. It is a position sensitive criterion based on the elastic stability. The goal of this criterion is to quantify and predict the early stages of the plasticity or the onset of the permanent deformation. 
As an energetic, atomic scale criterion, " $\wedge$-criterion" is a successful criterion to perform a correct study of the nucleation of discrete dislocations in fcc metals [9]. It remains a fully continuum method and its implementation in a standard finite element software package requires only that the user supply the exact Cachy-Born constitutive relation by incorporating large strain constitutive relations provided directly by interatomic potentials. This criterion has been validated by both experimental methods and simulation methods. Figure 2.17 shows the displacement-controlled response, the dips correspond to elastic instabilities that can be predicted by this free-energy-based, position-dependent criterion.

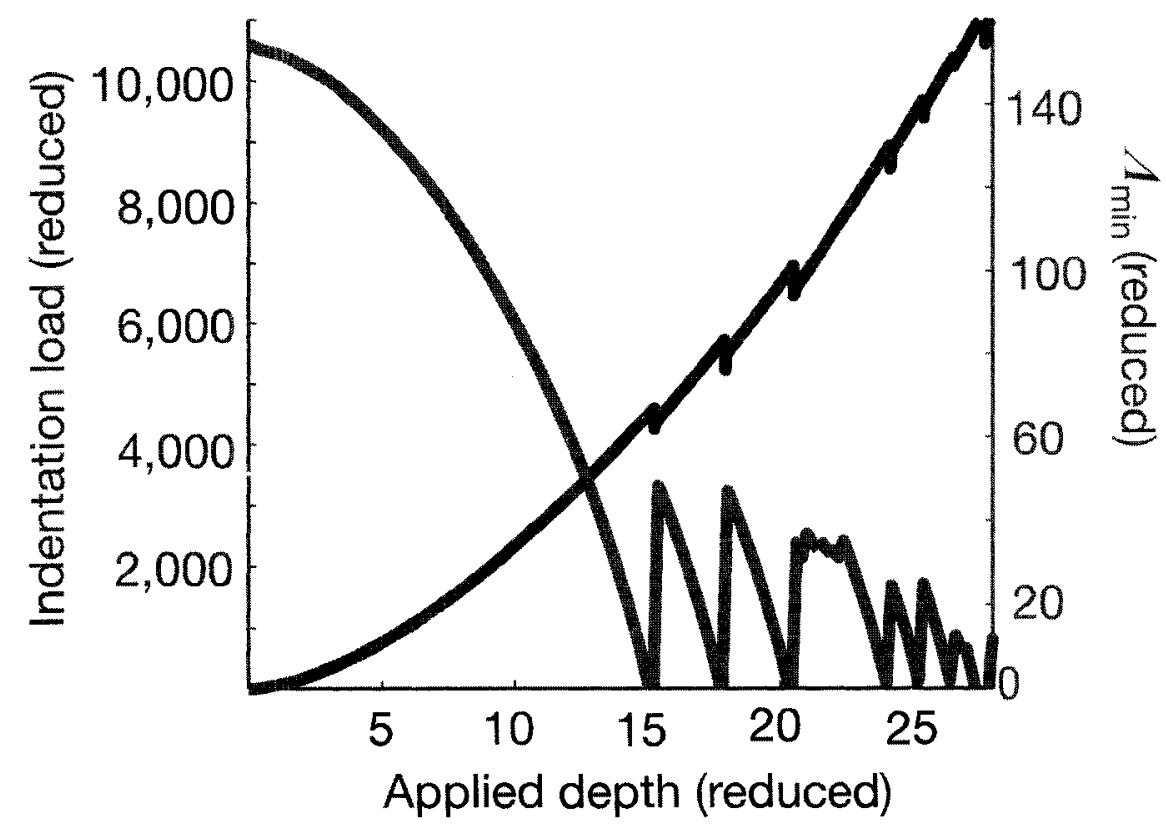

Figure 2.17: The correlation of load drops with a sequence of observed homogeneous dislocation dipole nucleation events. The elastic instability criterion $\wedge_{\min }$ tends to zero for the first several nucleation events. (Taken from [9]) 


\subsection{3 "Acharya/Miller" criterion}

Recently, a so-called "Acharya/Miller" criterion [1], was derived from the consideration of thermo-mechanics to predict the mechanical conditions which lead to dislocation nucleation. The basic idea of the criterion is that dislocation nucleation of a particular character depends upon a quantity, $\mathrm{N}_{m, l}$, defined as

$$
\mathrm{N}_{m, l}=|\mathbf{m} \cdot \operatorname{curl} \mathbf{T} \cdot \mathbf{l}|=\left|m_{i} e_{r j k} \mathrm{~T}_{i k, j} l_{r}\right|
$$

where, $\mathbf{m}$ is the unit in the direction of Burgers vector, $\mathbf{l}$ is the unit line direction, and $\operatorname{curl} \mathbf{T}$ is the curl of the stress tensor.

The criterion is used to predict the nucleation event of a specific dislocation (defined by its Burgers vector and line direction) at a point in the crystal where $\mathrm{N}_{m, l}$ for that dislocation type first reaches $\mathrm{N}_{\text {crit }}$ (A critical, material specific value).

To test whether this criterion accurately predicts dislocation nucleation it is necessary to evaluate $\mathrm{N}_{m, l}$ at each point in the crystal for each possible combination of line direction and Burgers vector. The largest value of $\mathrm{N}_{m, l}$ at each point in the crystal must be compared to the corresponding value at all the other points. Ultimately, the location and magnitude of the largest of all possible $\mathrm{N}_{m, l}$ values will identify the predicted nucleation site and dislocation type. As well, the maximum value of $\mathrm{N}_{m, l}$ just prior to nucleation will quantify the material property $\mathrm{N}_{c r i t}$ for this material.

The "Acharya/Miller" criterion had been tested and its ability to correctly predict dislocation nucleation was demonstrated via $2 \mathrm{D}$ atomistic simulations of the deformation of perfect crystal. Such simulations were performed using the CADD (coupled atomistics and discrete dislocation) method whereby a fully atomistic region is coupled to a larger linear elastic region [34]. Figure 2.18 shows the accurate location of dislocation nucleation for fcc Ni nanoindentation. As shown in the figure, 
locations of the nucleated defects are approximately indicated in the right-hand part of the figure by the inverted "T" symbols, which were originally nucleated at the positions of the circles. Correspondingly, Figure 2.19 shows the result of dislocation nucleation predictions. The circles shown in the figure indicate the actual locations of defect nucleation, demonstrating that the Schmid-like criterion is not an accurate predictor of nucleation. However, the stress-gradient based criterion accurately predicts the location and the type of nucleated defect. In this thesis, these tests are extended to more complex and rigorous cases of $3 \mathrm{D}$ indentation into crystals of various orientations.

\section{[FCCNi lndentation}

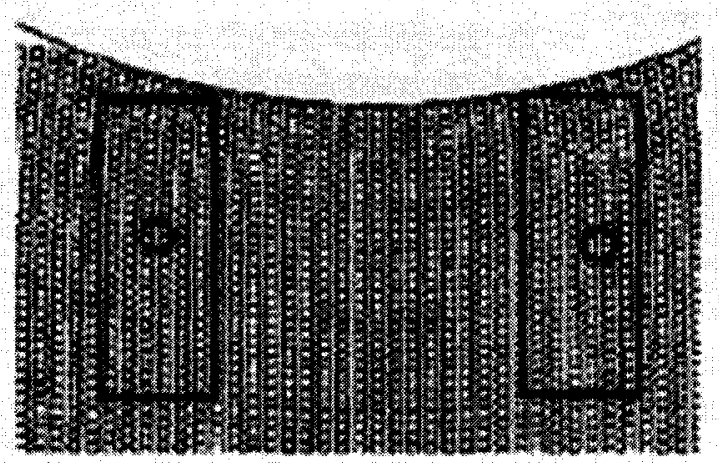

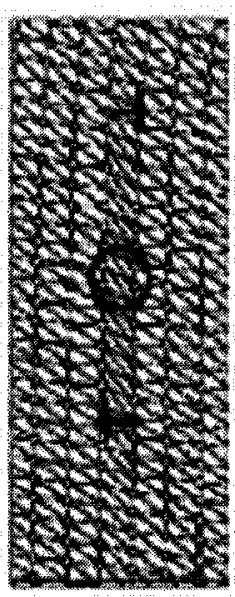

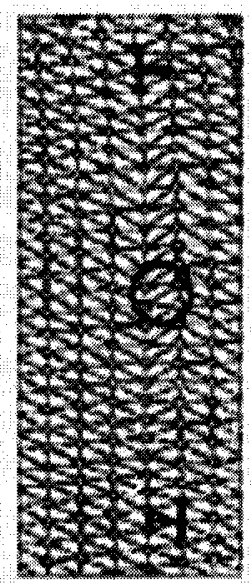

Figure 2.18: Dislocation nucleation. Snapshots of the nucleated defects in the atomistic region. The boxes in the figures of the left column are presented in close-up in the figures on the right. (Taken from [1]) 

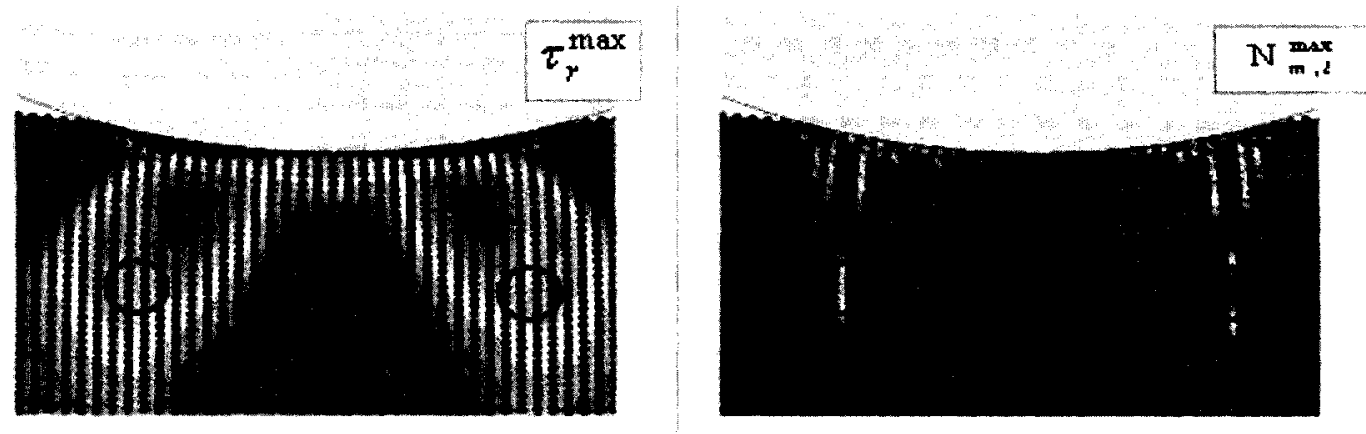

Figure 2.19: Predictions of dislocation nucleation. Comparison of the maximum Schmid stress in the left column with the maximum value of the stress-gradient based criterion in the right column. (Taken from [1]) 


\section{Chapter 3}

\section{Simulation tools and computational procedure}

\subsection{Simulation method}

The MD method is used to simulate nanoscale plasticity in order to understand the mechanisms of dislocation nucleation with nano-indentation simulations at 0K temperature. The model is built by making an initially perfect single crystal structure (i.e. a thin film of fcc crystal structure), then, a load is incrementally applied with a hard-sphere indenter through fixed displacement boundary conditions. In current work, the indentation is simulated by pushing the indenter to contact with metal substrate with a velocity of $0.05 \AA / p \sec (0.5 \mathrm{~m} / \mathrm{s})$. The atoms are modeled by the empirical interatomic potentials based on the EAM (Embedded Atom Method) potential. The indenter is modeled as a fictitious atom with a large specific radius, a repulsive potential is introduced to simulate the interaction between the indenter and its neighbor atoms.

\subsubsection{Description of Nano-indentation modeling}

Three different surfaces ( $(001),(110)$ and (111)) for two fcc metals ( $\mathrm{Cu}$ and $\mathrm{Ni}$ ) are considered and used to test the capability of the "Acharya/Miller" criterion to correctly predict the location and moment of the initial plastic deformation. The model of nano-indentation is simulated within the framework of MD method using 
the "dynamo" code [35] in a three-dimensional Cartesian coordinate system. First, the single fcc crystal structure is built by representing each atom in the lattice by its three dimensional coordinates, and a simulation box is defined in which the atoms reside.

After the simulation cell is created, the boundary conditions are applied to the model. The periodic boundary conditions (PBC) are set to the four side surfaces ( $x \& y$ directions) to avoid the surface effects. In other words, the cubical simulation box is replicated throughout the $x \& y$ plane to form an infinite crystal. In the course of the simulation, if an atom leaves the central simulation box, one of its images will enter through the opposite face. Therefore, there are no walls at the boundary of the central box, and the system has no surfaces except the free surface to be indented and the fixed bottom surface. The number of particles in the central box (and hence in the entire system) is conserved. The visualization of periodic boundary conditions is shown in Figure 3.1.

To obtain the equilibrium atomic crystal structure before the displacement controlled MD simulation, the conjugate gradient method is used for the energy minimization to realize the system relaxation. Ultimately, a lattice constant in the equilibrium state is obtained and will be used in the following dislocation calculations. Here, the detailed information of three simulation models with different orientations (separately rotating the standard fcc crystal structure to make the indentation surface either a (001), (110) or (111) plane) is introduced. 


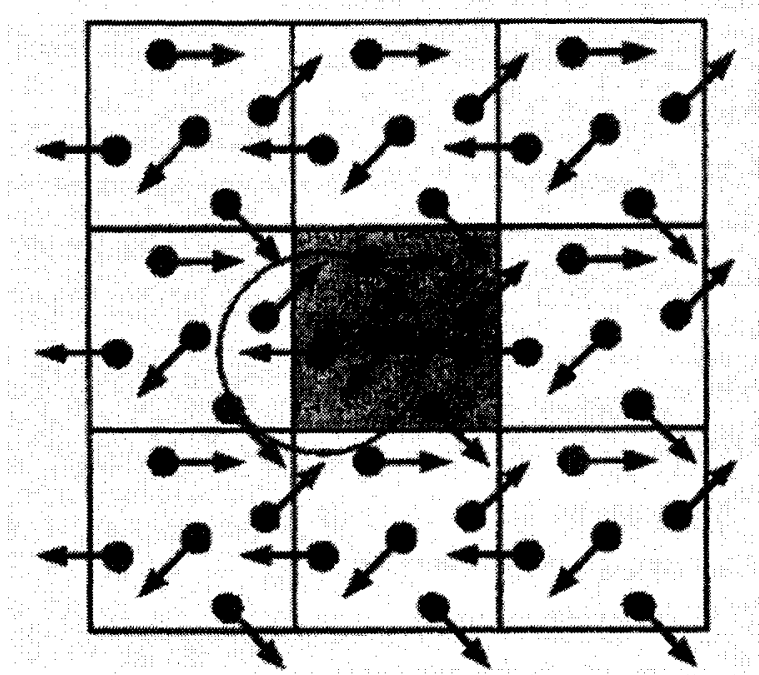

Figure 3.1: Showing the periodic boundary positions of atoms. The shaded square (unit cell) has periodic copies all around it. (Taken from [36])

\section{(001) orientation system}

A three-dimensional atomic configuration of the $C u(001)$ or $N i(001)$ model is shown in Figure 3.2. The metal substrate is modeled as a slab (approximately $72 \times 72 \times 54 \AA^{3}$ in the $x, y$ and $z$ directions respectively) containing a total of 24000 atoms with periodic boundary conditions paralleling to the side surfaces. The indentation surface is traction free and the bottom atomic layer is fully constrained. The orientation of the slab is such that the directions [100], [010] and [001] coincide with $x, y$ and $z$ axes.

\section{(110) orientation system}

A three-dimensional atomic configuration of the $C u(110)$ or $N i(110)$ model is modeled as a slab (approximately $77 \times 73 \times 52 \AA^{3}$ in the directions of $x, y$ and $z$ respectively) containing a total of 24000 atoms with periodic boundary conditions paralleling to the side surfaces. Similarly, the indentation surface (110) is traction free and the bottom atomic layer is fully constrained. The orientation of the slab is such that the directions [111], [112] and [110] coincide with $x, y$ and $z$ axes. 


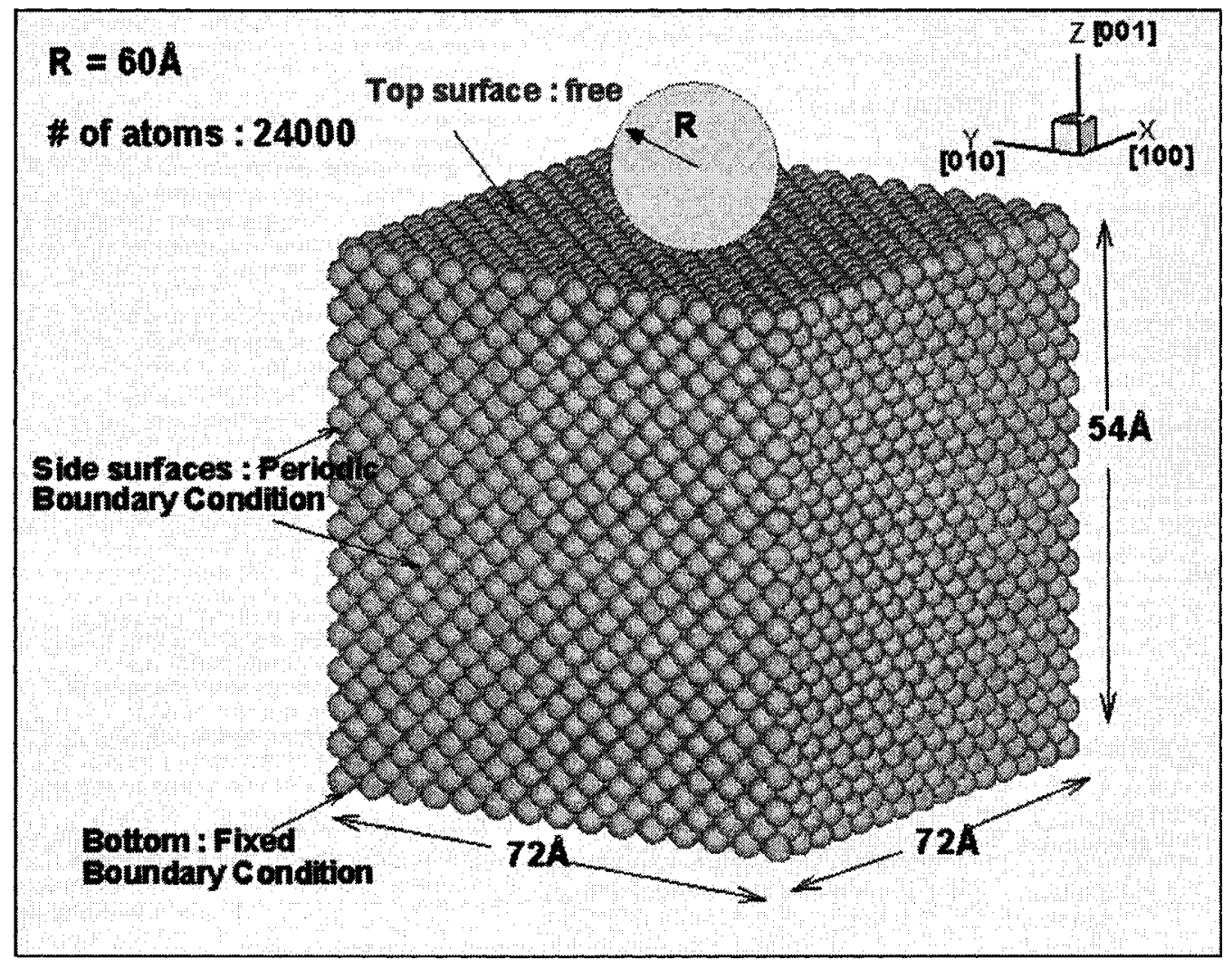

Figure 3.2: Atomic configuration of indenter and $\mathrm{Cu}$ or Ni substrate, indentation surface is (001) plane. 


\section{(111) orientation system}

A three-dimensional atomic configuration of the $C u(111)$ or $N i$ (111) model is modeled as a slab (approximately $67 \times 67 \times 63 \AA^{3}$ in the directions of $x, y$ and $z$ respectively) containing a total of 23400 atoms with periodic boundary conditions paralleling to the side surfaces. The indentation surface (111) is traction free and the bottom atomic layer is fully constrained. The orientation of the slab is such that the directions $[1 \overline{1} 0],[11 \overline{2}]$ and $[111]$ coincide with $x, y$ and $z$ axes.

\subsection{Testing of the "Acharya/Miller" criterion}

The requirement of the "Acharya/Miller" criterion is to calculate a scalar $\mathrm{N}_{m, l}$ for every atom in the atomic system and the major task of current calculation is to obtain the stress gradient from known stresses (the result of MD simulations) and coordinates of atoms in three-dimensional space. Firstly, the atomic level stresses should be calculated based on EAM potential and indenter introduced potential using the MD method, then the stress-gradient for every atom is obtained with LOPI (local optimal point interpolating) [37] method and the accuracy is verified with the solution of Hertz theory, ultimately, the $\mathrm{N}_{m, l}$ values are computed to test the current criterion.

\subsubsection{Potential between the indenter and its neighbor atoms}

In this MD simulation system, the displacement controlled nanoindentation is conducted by positioning a one-atom spherical indenter with a specific radius (60 ̊), modeled by a repulsive potential, into the surface of the atomistic sample. This is achieved by introducing a repulsive interatomic potential between the indenter and its neighborhood atoms (where the distance from the indenter center to the atom center is less than the radius of indenter). Thus, the indenter effectively repels these 
atoms, behaving approximately like a rigid and frictionless sphere. The repulsive potential is described by the equation:

$$
\Psi\left(r_{I j}\right)= \begin{cases}C\left(R-r_{I j}\right)^{2}, & r_{I j}<R \\ 0 & r_{I j}>R\end{cases}
$$

where $C$ is a constant related to the effective stiffness of the indenter and is set to $C=1 \mathrm{eV} / \stackrel{\circ}{\mathrm{A}}^{2}$ in current simulations. $R$ is the radius of the indenter and $r_{I j}$ is the distance from the center of the indenter to the center of the $j$ th atom. Two values of $R, 30 \AA$ and $60 \AA$, are used. Note that, the evolutions of dislocation activities corresponding to different indenter radii are found to be very similar for a specific crystal orientation.

The interaction between the indenter and its neighbor atoms will change the system energy shown as the equation 3.3:

$$
\begin{aligned}
& \mathrm{E}_{I}=\sum_{j} \Psi\left(r_{I j}\right) \\
& \mathrm{E}=\sum_{i=1}^{N} \mathrm{E}_{i}+\mathrm{E}_{I}
\end{aligned}
$$

The first term $\sum_{i=1}^{N} \mathrm{E}_{i}$ is regarded as the EAM potential, the second term $\mathrm{E}_{I}$ is the sum of potentials between the spherical indenter and its neighbor atoms.

\subsubsection{Calculation of atomic level stresses}

In current atomic simulation, the atomic level stress is based on the EAM potential and the repulsive potential. Specially, the atomic stress of indenter neighbors is the superposition of these two part of stress, the first part is the stress evaluated from the EAM potential and the second part is the stress introduced by the repulsive potential of the indenter. For other atoms in the atomic system away from the indenter, the stress is represented only by the EAM term. 


\section{Part 1: Atomic level stress evaluated from the EAM potential}

The change of energy due to the deformation to the first order in the strain tensor is given by [38]:

$$
\Delta \mathrm{E}=\sum_{i} \sum_{j \neq i}\left\{\frac{1}{2}\left[\frac{\partial \mathrm{E}}{\partial \varepsilon_{i j}^{I J}}\right]_{\varepsilon=0} \varepsilon_{i j}^{I J}\right\}
$$

where the superscripts $I$ and $J$ refer to Cartesian components, and $\varepsilon_{i j}^{I J}$ is the strain between atoms $i$ and $j$. The notation $\varepsilon=0$ implies that the quantity in the square brackets is evaluated for all strains equal to zero. For the special case where the strain tensor is constant throughout the body, that means all $\varepsilon_{i j}^{I J}=\varepsilon^{I J}$, this expression can be re-written in the simple form:

$$
\Delta \mathrm{E}=\sum_{i} \Omega_{i} \sigma_{i}^{I J} \varepsilon^{I J}
$$

where $\Omega_{i}$ is the atomic volume at site $i$, and the atomic level stresses are defined as:

$$
\sigma_{i}^{I J}=\frac{1}{\Omega_{i}} \sum_{j \neq i}\left[\frac{\partial \mathrm{E}}{\partial \varepsilon_{i j}^{I J}}\right]_{\varepsilon=0}
$$

where $\sigma_{i}^{I J}$ refers to the stress tensor at atom $i$.

Ultimately, this is commonly known as virial definition of atomic stresses, the partial derivative in this expression can be evaluated from EAM [32] to give,

$$
\sigma_{i}^{r J}=\frac{1}{\Omega_{i}} \sum_{j \neq i}\left[V^{\prime}\left(r_{i j}\right)+F^{\prime}\left(\bar{\rho}_{i}\right) \rho^{\prime}\left(r_{i j}\right)+F^{\prime}\left(\bar{\rho}_{j}\right) \rho^{\prime}\left(r_{i j}\right)\right] \frac{r_{i j}^{I} r_{i j}^{J}}{r_{i j}}
$$

where $r_{i j}^{I}$ is the $I$ th component of the relative position vector between atoms $i$ and $j, r_{i j}$ is its magnitude, and the remaining quantities are as previously defined for EAM. 


\section{Part 2: stress introduced by repulsive potential of indenter}

After introducing the indenter, the force on indenter neighbor atom $i$ becomes:

$$
\begin{aligned}
& \vec{f}_{i}=-\frac{\partial \mathrm{E}_{I}}{\partial \vec{x}_{i}}=-\frac{\partial \Psi\left(r_{I i}\right)}{\partial \vec{x}_{i}}=-\Psi^{\prime}\left(r_{I i}\right) \frac{\partial r_{I i}}{\partial \vec{x}_{i}} \\
& \Psi\left(r_{I i}\right)=C\left(R-r_{I i}\right)^{2} \\
& \Psi^{\prime}\left(r_{I i}\right)=-2 C\left(R-r_{I i}\right) \\
& r_{I i}=\sqrt{\left(\vec{x}_{I}-\vec{x}_{i}\right) \cdot\left(\vec{x}_{I}-\vec{x}_{i}\right)}
\end{aligned}
$$

where, $\vec{x}_{i}$ is the position of atom $i$, which is the indenter's neighbor; $R$ is the radius of indenter; $r_{I i}$ is the distance from the center of indenter to atom $i . \bar{f}_{i}$ is the atomic force introduced by the indenter to its neighbor atom $i$.

Substituting Equation 3.10 into 3.8, the force between indenter and its neighbor atom $i$ is given by:

$$
\vec{f}_{i}=2 C\left(R-r_{I i}\right) \frac{\partial r_{I i}}{\partial \vec{x}_{i}}
$$

In order to obtain the atomic level stress introduced by the indenter to its neighbor atoms, a traction $t_{i}^{\mathrm{K}}$ shown in Figure 3.3 along the direction of $\vec{f}_{i}$ is defined and can

be obtained by dividing force $\vec{f}_{i}$ with the contact area $A_{i}$ between indenter and a specific atom $i$.

$$
\begin{aligned}
& \mathbf{t}_{i}^{K}=\frac{\vec{f}_{i}}{A_{i}}=\frac{2 C}{A_{i}}\left(R-r_{I i}\right) \frac{r_{I i}^{K}}{r_{I i}} \\
& A_{i}=\pi \cdot r_{i}^{2}=\frac{\pi \cdot a_{0}^{2}}{8}
\end{aligned}
$$

where, $a_{0}$ is the lattice constant for a specific fcc metal crystal. $r_{i}$ is the radius of contact area between the indenter and an individual atom. 
The atomic level stress is then found from,

$$
\mathbf{t}_{i}^{\mathrm{K}}=\sigma_{i}^{\mathrm{K} J} \mathbf{r}^{J}
$$

where, $\mathbf{r}^{J}$ is the surface normal.

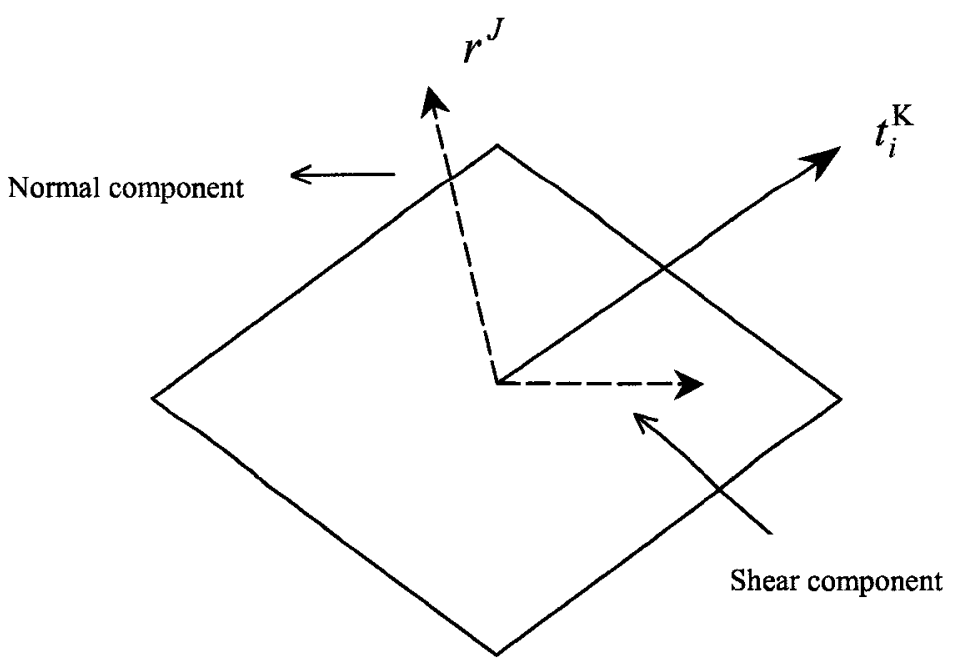

Figure 3.3: The definition of traction $\mathbf{t}_{i}^{\mathrm{K}}$.

To verify the accuracy of the atomic stress, the results of stresses calculated by the MD method and Hertz theory are compared for small indentation depth and found that stress formula given in current work are efficient with high quality. This gives confidence that the atomic stress used to test the "Acharya/Miller" criterion are correct. The atomic level stress just prior to the critical nucleation event will be computed later to verify the criterion of dislocation nucleation below the indenter.

\subsubsection{Computation of stress gradient with LOPI method}

Here, an interpolation method is used as a tool to calculate the stress gradient within a meshfree framework using the recently developed LOPI [37] scheme. This method 
is significantly faster than the interpolation of the finite element method due to the elimination of mesh generation, and can be easily linked to a CAD system. The LOPI method yields not only continuous stress fields but also continuous stress gradients. However, in FEM, the first order derivative of stresses are continuous only inside the element but discontinuous between the adjacent elements. Therefore, the LOPI method is more suitable for current task than the FEM interpolation.

Here, the meshfree approximation and interpolation are constructed among a set of scattered particles that have no particular topological connections among them. The scattered data interpolation problem can be defined by giving a set of $n$ irregularly distributed points:

$$
P_{i}=\left(x_{i}, y_{i}, z_{i}\right), \quad i=1, \cdots \cdots, n
$$

The scalar values $F_{i}$ associated with each point satisfy $F_{i}=F\left(x_{i}, y_{i}, z_{i}\right)$. For some underlying function $F(x, y, z)$, an interpolating function $\bar{F} \approx F(x, y, z)$ is obtained such that for $i=1, \cdots \cdots, n$

$$
\bar{F}\left(x_{i}, y_{i}, z_{i}\right)=F_{i}
$$

In current work, the scattered points $P_{i}$ are selected from the neighbors of the evaluated atom within the cut-off radius, and they distribute around the represented atom. All these requirements make the function $F$ a multivariate function that defines a smooth bended shell surface in three-dimensional space (e.g., $F=a x^{2}+$ $\left.b y^{2}+c z^{2}+d x y+e y z+f x z+h\right)$. The basic ideas of LOPI is to use a least-square polynomial approximation based on a set of scattering points, and then correcting the difference between the real values and the least-square approximation values with multiquadrics interpolation. The following two sections are simple introductions to 
the algorithms of "Least-squares polynomial approximation" and "Multiquadrics interpolation".

\section{Least-squares polynomial approximation}

A Least-squares polynomial approximation is used to find a continuous function for a group of scattered points. The approximation function should fit the profile distribution of these scattered points, but will not exactly pass through the scattered points. LOPI uses a polynomial approximation to obtain the distribution configuration of stresses decided by the stresses of neighbor atoms for a specific atom [37].

It is possible to use any polynomial of $m$ degree to fit experimental data (provided that $n \geq m+1)$ :

$$
\left(x_{1}, y_{1}, z_{1}, f_{1}\right),\left(x_{2}, y_{2}, z_{2}, f_{2}\right), \cdots,\left(x_{n}, y_{n}, z_{n}, f_{n}\right)
$$

For least-squares polynomial approximation, a polynomial approximation model $\varphi$ is given by:

$$
\varphi(x, y, z)=\sum_{i=0}^{m} c_{i}(x, y, z) p_{i}(x, y, z)=\mathbf{p}^{\mathrm{T}}(x, y, z) \mathbf{c}(x, y, z)
$$

where, $m$ is the number of terms in the basis, $p_{i}(x, y, z)$ are monomial basis functions, and $c_{i}(x, y, z)$ are their coefficients, which as indicated are functions of the spatial coordinates $x, y, z$ because the approximation varies from atom to atom in the crystal with LOPI.

In 3D, examples of commonly used bases are the linear basis:

$$
\mathbf{p}^{\mathrm{T}}=(1, x, y, z)
$$

And the quadratic basis:

$$
\mathbf{p}^{\mathrm{T}}=\left(1, x, y, z, x^{2}, y^{2}, z^{2}, x y, y z, x z\right)
$$


Assuming the quadratic function $I(x, y, z)$ is given by:

$$
I(x, y, z)=\sum_{i=0}^{m}\left[f_{i}-c_{i}(x, y, z) p_{i}(x, y, z)\right]^{2}
$$

The goal of the approximation is to minimize the difference between the value of approximation function and experimental data on the scattered points, the following formula is regarded as the prerequisite which makes $x, y, z$ reach to the minimum value.

$$
\frac{\partial I}{\partial c_{k}}=2 \sum_{i=0}^{m}\left[f_{i}-c_{i}(x, y, z) p_{i}(x, y, z)\right] p_{k}(x, y, z)=0, \quad k=0,1, \cdots, n
$$

The above formula could be written as a group of equations of linear algebra based on coefficients $c_{0}, c_{1}, \cdots, c_{n}$,

$$
\sum_{j=0}^{n}\left(\varphi_{j}, \varphi_{k}\right) c_{j}=\left(\mathbf{f}, \varphi_{k}\right) \quad k=1,2, \cdots, m
$$

where, $\varphi_{j}=\left(\varphi_{j}\left(x_{0}, y_{0}, z_{0}\right), \varphi_{j}\left(x_{1}, y_{1}, z_{1}\right), \cdots, \varphi_{j}\left(x_{m}, y_{m}, z_{m}\right)\right), \mathbf{f}=\left(f_{0}, f_{1}, \cdots, f_{m}\right)$, $(\cdot, \cdot)$ indicates the dot product.

These linear equations can be solved and obtain the unique solution of $c_{0}, c_{1}, \cdots, c_{n}$, then, the expression of polynomial approximation function $\varphi(x, y, z)$ can be obtained.

Figure 3.4 shows the visualization of least-square approximation based on a set of scattered points on a one-dimensional line. Note that the curve does not exactly pass through the points and LOPI uses multiquadrics to correct this small error. 


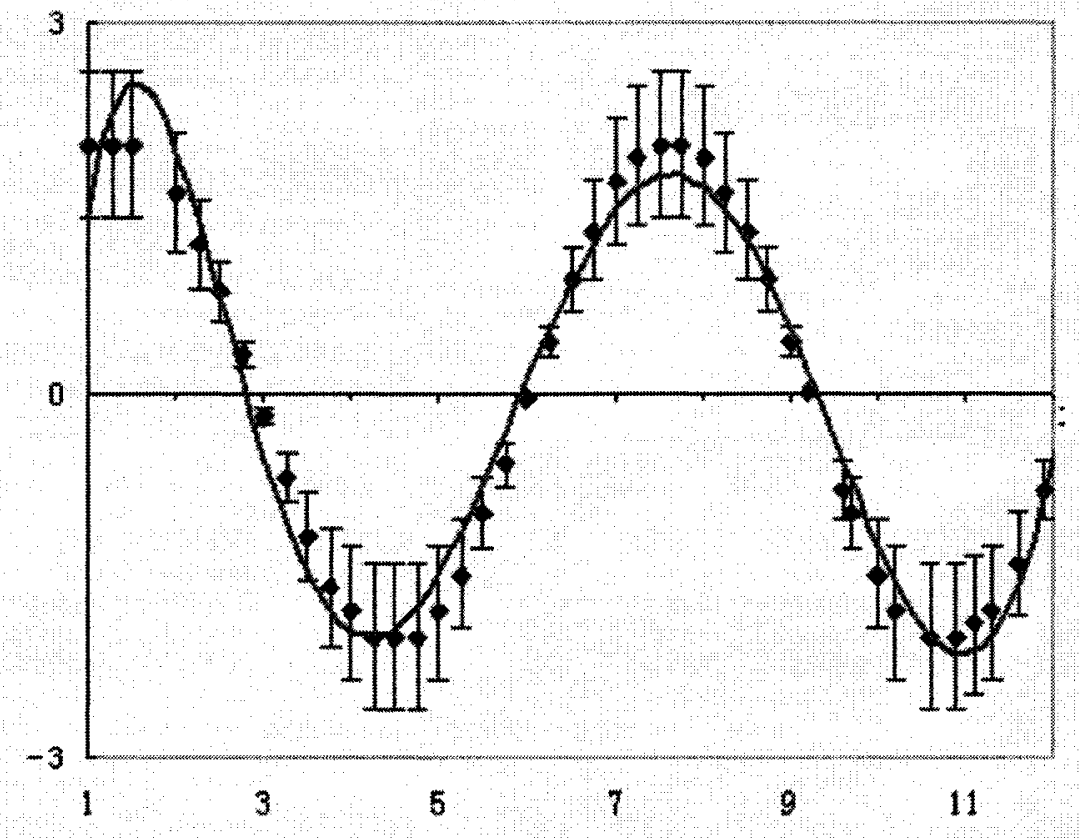

Figure 3.4: An example curve of one-dimensional approximation with least-square method based on a set of scattered points. 


\section{Multiquadrics interpolation}

Multiquadrics [39] is one of important methods of interpolation, for example, in three-dimensional space, an interpolating function $F(x, y, z)$ of $n$ scattered points $\left(x_{k}, y_{k}, z_{k}\right)$ is a combination of basis functions $g_{k}$ and is defined by:

$$
F(x, y, z)=\sum_{k=1}^{n} a_{k} g_{k}(x, y, z)
$$

where the coefficients $a_{k}$ are determined from the interpolation requirements $F_{k}=$ $F\left(x_{k}, y_{k}, z_{k}\right)$. There are many choices for the basis $g_{k}$, a popular choice is the radial basis function:

$$
\begin{aligned}
& g_{k}(x, y, z)=g_{k}\left(r_{k}\right) \\
& r_{k}=\left\|(x, y, z)-\left(x_{k}, y_{k}, z_{k}\right)\right\|_{2}=\sqrt{\left(x-x_{k}\right)^{2}+\left(y-y_{k}\right)^{2}+\left(z-z_{k}\right)^{2}}
\end{aligned}
$$

The Multiquadrics interpolation used in LOPI method is given by:

$$
g_{k}\left(r_{k}\right)=\sqrt{1+c_{q} r_{k}^{2}}, \quad c_{q}>0
$$

An exact interpolation of data $\mathbf{f}$ as the linear combination $\sum_{k=1}^{n} a_{k} g_{k}\left(r_{k}\right)$ may be constructed by computing the coefficients $a_{k}$ using:

$$
\mathbf{a}=\mathbf{V}^{-1} \mathbf{f}
$$

where $\mathbf{f}=\left[F_{1}, F_{2}, \cdots, F_{n}\right]^{\mathrm{T}}, \mathbf{a}=\left[a_{1}, a_{2}, \cdots, a_{n}\right]^{\mathrm{T}}$

And the Vandermondian (or Grammian) $\mathbf{V}$ is given:

$$
\mathbf{V}=\left[\begin{array}{lll}
g\left(x_{1}-x_{1}\right) & \cdots & g\left(x_{k}-x_{1}\right) \\
\vdots & \ddots & \vdots \\
g\left(x_{1}-x_{k}\right) & \cdots & g\left(x_{k}-x_{k}\right)
\end{array}\right]
$$


To find the corresponding interpolation, the coefficients a must be solved for this system. Figure 3.5 shows an visualized example of Multiquadrics method in onedimensional space, interpolating the error between the data and the least-squares fit shown in Figure 3.4. The sum of the least squares function and the multiquadrics interpolation define the LOPI method. In current work, the LOPI method is used to obtain a 3D smooth stress shell which is very convenient to calculate the stress gradient of the presented atom.

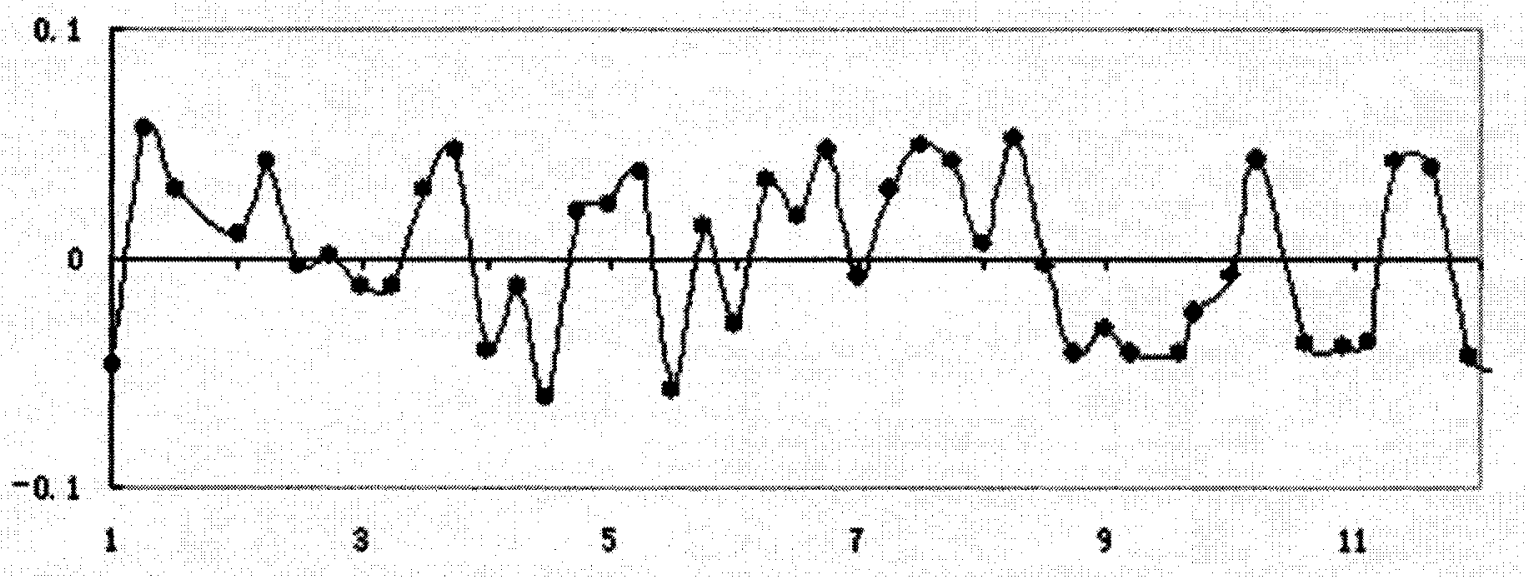

Figure 3.5: An interpolation example using Multiquadrics method based on the difference between approximation and real value.

\section{Validating with Hertz Theory}

To test the efficiency and accuracy of stress gradient calculation with LOPI method, the LOPI result is compared with the standard solution of contact mechanics (Hertz theory) [40]. Under Hertzian contact between an axisymmetric (spherical) tip with radius $R$ and a flat substrate, the relationship between the indenter load $P$ and its displacement $\delta$ is given as equation 3.31 .

$$
\mathrm{P}=\frac{4}{3} \delta^{3 / 2} R^{1 / 2} \mathrm{E}^{*}
$$


The radius of the contact circle is:

$$
a=\left(\frac{3 P R}{4 \mathrm{E}^{*}}\right)^{1 / 3}
$$

The displacement of indenter is:

$$
\delta=\frac{a^{2}}{R}=\left(\frac{9 P^{2}}{16 R E^{* 2}}\right)^{1 / 3}
$$

where, $\mathrm{E}^{*}$ is the reduced or combined tip/substrate modulus and given by:

$$
\frac{1}{\mathrm{E}^{*}}=\frac{1-\gamma_{t i p}^{2}}{\mathrm{E}_{t i p}}+\frac{1-\gamma_{s u b}^{2}}{\mathrm{E}_{s u b}}
$$

With the above equations, it is possible to predict the load-displacement curve in the elastic regime and to estimate the contact radius to first order.

According to the Hertz theory, the pressure which is exerted between two contracting frictionless elastic solids of revolution is given by:

$$
p(r)=p_{0}\left(a^{2}-r^{2}\right)^{1 / 2} / a
$$

where, $r$ is the distance from the $z$ axis to a specific point $\left(r^{2}=x^{2}+y^{2}\right), p_{0}$ is the maximum pressure on the contact circle, and $a$ is the contact radius.

\section{Distribution of Hertz stress}

Within the framework of a cylinder coordinate system, the stresses and displacements can be produced by the circular distributed point contacts acting normally to the surface and are given by [2], [40]: 

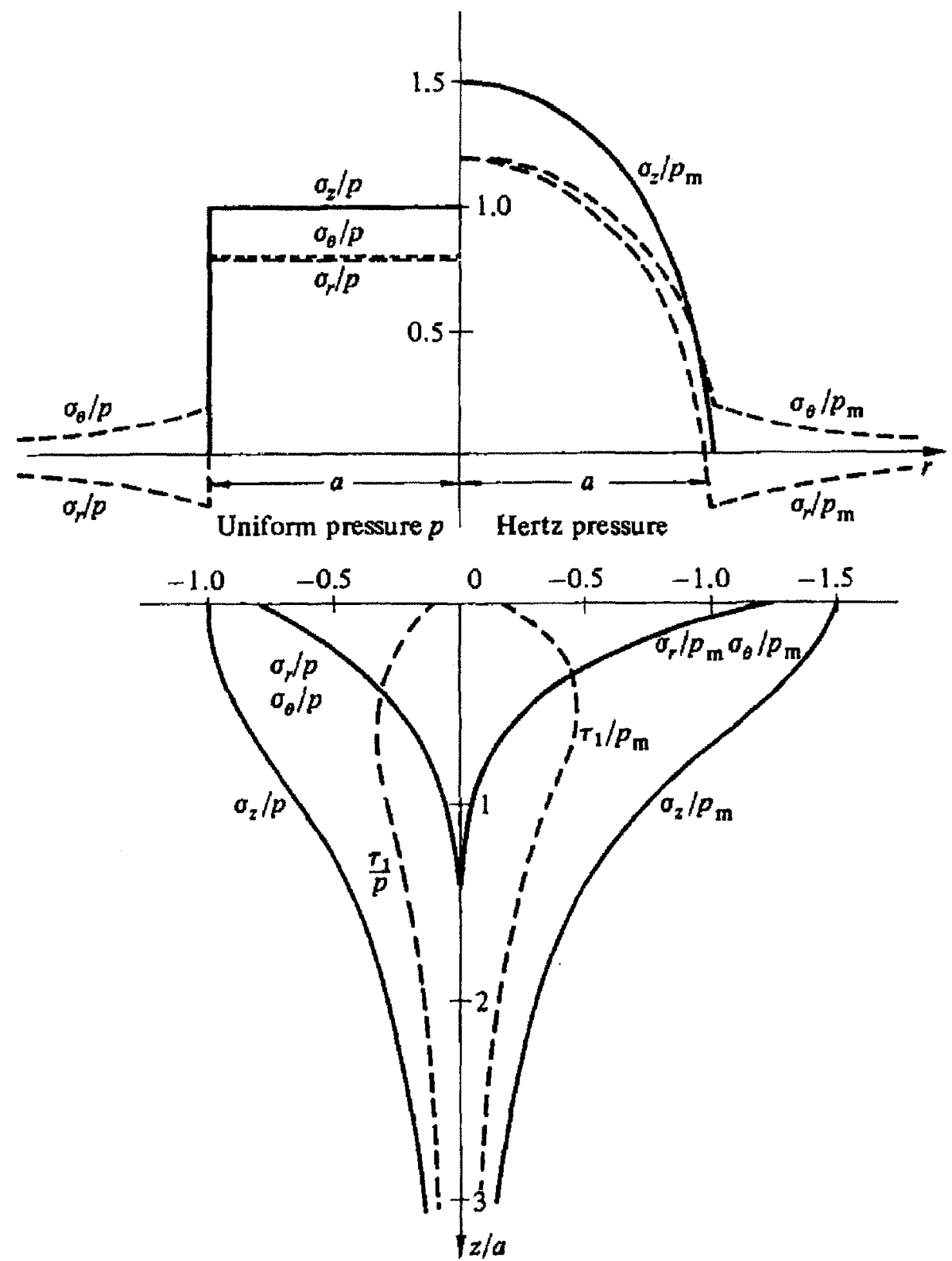

Figure 3.6: Stress distributions at surface and along the axis of symmetry caused by (left) uniform pressure and (right) Hertz pressure acting on a circular area radius $a$. (Taken from [40]) 
Stress on the surface $(z=0)$

$$
\begin{array}{ll}
\frac{\bar{\sigma}_{r}}{p_{0}}=\frac{1-2 \nu}{3}\left(\frac{a^{2}}{r^{2}}\right)\left\{1-\left(1-\frac{r^{2}}{a^{2}}\right)^{\frac{3}{2}}\right\}-\left(1-\frac{r^{2}}{a^{2}}\right)^{\frac{1}{2}}, \quad r \leq a \\
\frac{\bar{\sigma}_{\theta}}{p_{0}}=-\frac{1-2 \nu}{3}\left(\frac{a^{2}}{r^{2}}\right)\left\{1-\left(1-\frac{r^{2}}{a^{2}}\right)^{\frac{3}{2}}\right\}-2 \nu\left(1-\frac{r^{2}}{a^{2}}\right)^{\frac{1}{2}}, \quad r \leq a \\
\frac{\bar{\sigma}_{z}}{p_{0}}=-\left(1-\frac{r^{2}}{a^{2}}\right)^{\frac{1}{2}}, & r \leq a
\end{array}
$$

and:

$$
\begin{array}{ll}
\frac{\bar{\sigma}_{r}}{p_{0}}=-\frac{\bar{\sigma}_{\theta}}{p_{0}}=(1-2 \nu) \frac{a^{2}}{3 r^{2}}, & r>a \\
\frac{\bar{\sigma}_{z}}{p_{0}}=-\left(1-\frac{r^{2}}{a^{2}}\right)^{\frac{1}{2}}, & r>a
\end{array}
$$

Stresses below the top surface

$$
\begin{aligned}
\frac{\sigma_{r}}{p_{m}}= & \frac{1-2 \nu}{2} \frac{a^{2}}{r^{2}}\left[1-\left(\frac{z}{u^{\frac{1}{2}}}\right)^{3}\right]+\frac{3}{2}\left(\frac{z}{u^{\frac{1}{2}}}\right)^{3} \frac{a^{2} u}{u^{2}+a^{2} z^{2}}+ \\
& \frac{3 z}{2 u^{\frac{1}{2}}}\left[u \frac{1-\nu}{a^{2}+u}+(1+\nu) \frac{u^{\frac{1}{2}}}{a} \tan ^{-1}\left(\frac{a}{u^{\frac{1}{2}}}\right)-2\right] \\
\frac{\sigma_{\theta}}{p_{m}}= & \frac{2 \nu-1}{2} \frac{a^{2}}{r^{2}}\left[1-\left(\frac{z}{u^{\frac{1}{2}}}\right)^{3}\right]- \\
& \frac{3 z}{2 u^{\frac{1}{2}}}\left[2 \nu+u \frac{1-\nu}{a^{2}+u}-(1+\nu) \frac{u^{\frac{1}{2}}}{a} \tan ^{-1}\left(\frac{a}{u^{\frac{1}{2}}}\right)\right] \\
\frac{\sigma_{z}}{p_{m}}= & -\frac{3}{2}\left(\frac{z}{u^{\frac{1}{2}}}\right)^{3}\left(\frac{a^{2} u}{u^{2}+a^{2} z^{2}}\right) \\
\frac{\tau_{r z}}{p_{m}}= & -\frac{3}{2}\left(\frac{r z^{2}}{u^{2}+a^{2} z^{2}}\right)\left(\frac{a^{2} u^{\frac{1}{2}}}{a^{2}+u}\right)
\end{aligned}
$$

where, 


$$
\begin{aligned}
p_{m} & =\frac{2}{3} p_{0} \\
u & =\frac{1}{2}\left[\left(r^{2}+z^{2}-a^{2}\right)+\left[\left(r^{2}+z^{2}-a^{2}\right)^{2}+4 a^{2} z^{2}\right]^{\frac{1}{2}}\right]
\end{aligned}
$$

It should be noted that for $z=0$, and for values of $\frac{r}{a}<1$, the value for $u$ is always zero but the state of stress directly beneath the indenter may be calculated with reasonable accuracy by taking a sufficiently small value of $z$.

The theoretical stress distribution of Hertz theory within the framework of a cylinder coordinate system is shown as Figure 3.6. Observing the stress on the surface of contact from the top part of this figure, it is easily seen that $\sigma_{r}$ and $\sigma_{\theta}$ continuously decrease from the center to the circumference of contact circle inside the contact circle, whereas beyond this circle, the distribution of stress will change and gradually converge to zero. Similarly, the stress distribution along the $z$ axis also can be observed from the bottom part of Figure 3.6, it is clear that $\sigma_{r}$ and $\sigma_{\theta}$ gradually decrease with the increased depth and terminally convergent to zero. Such stress distributions also can be mapped into the framework of a Cartesian coordinate system by coordinate transformation, $\sigma_{x}$ and $\sigma_{y}$ should have the similar stress distribution as $\sigma_{r}$ because of the symmetry property.

To test the efficiency of the LOPI method for calculating of the stress gradient, Figure 3.7 shows the comparison of stress gradient distribution between the LOPI method and the Hertz theory (i.e., the derivative of Hertz stress described in the above section). It is seen that the two results agree with each other (the errors of stress gradient below the top surface are less than $2 \%$ ) except the atoms on the top surface, and such inaccuracy will be transfered to the calculation of $\mathrm{N}_{m, l}$. The surface error is introduced from a limitation of the meshless interpolation method 
of LOPI because the neighbor atoms of surface atoms on surface only come from a half space.

To quantitatively investigate the efficiency of the LOPI method applied throughout the crystal structure, a few columns of atoms from the top surface to the bottom are selected, then $\mathrm{N}_{m, l}$ value for these atoms are calculated separately with the LOPI method and Hertz solution. The $\mathrm{N}_{m, l}$ versus the depth of atom curves ( from Figure 3.8 to Figure 3.11 ) shows that the LOPI predicts spuriously large values of $\mathrm{N}_{m, l}$ near free surfaces, as shown in the figures, the dotted line indicates the LOPI method and Solid line indicates Hertz solution (stress derivative). The fact is shown that the LOPI method has high quality for the atomic region below top surface, after the 2nd atomic layer. Inset shows an enlargement of the main plot. the LOPI method still can be used in current tests, but must be taken care to avoid using results from the surface layer. 


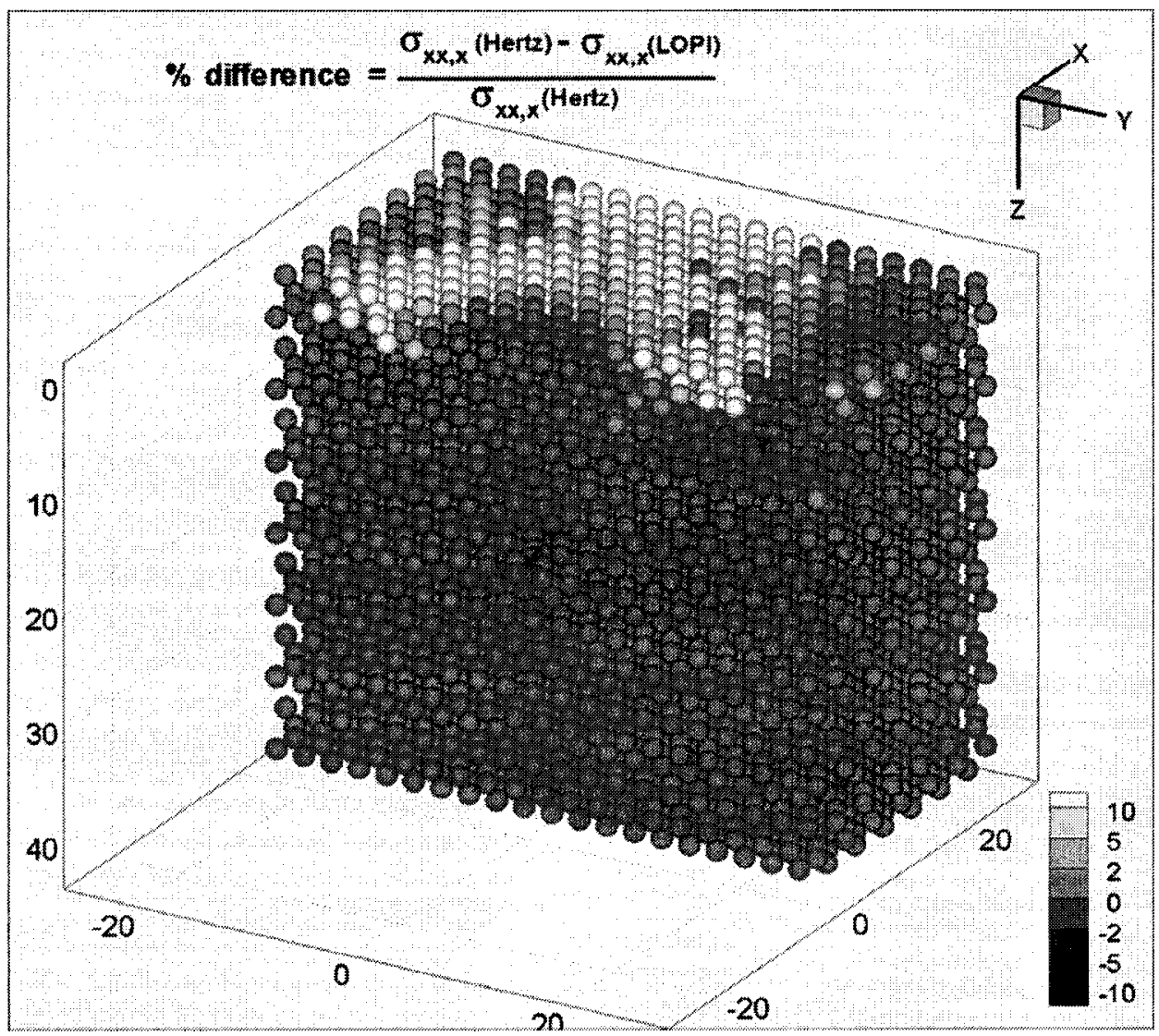

Figure 3.7: Difference of stress gradient calculated by Hertz theory and LOPI method based on the same atomic stresses and coordinates. The two results agree with each other except the atoms on top surface. 


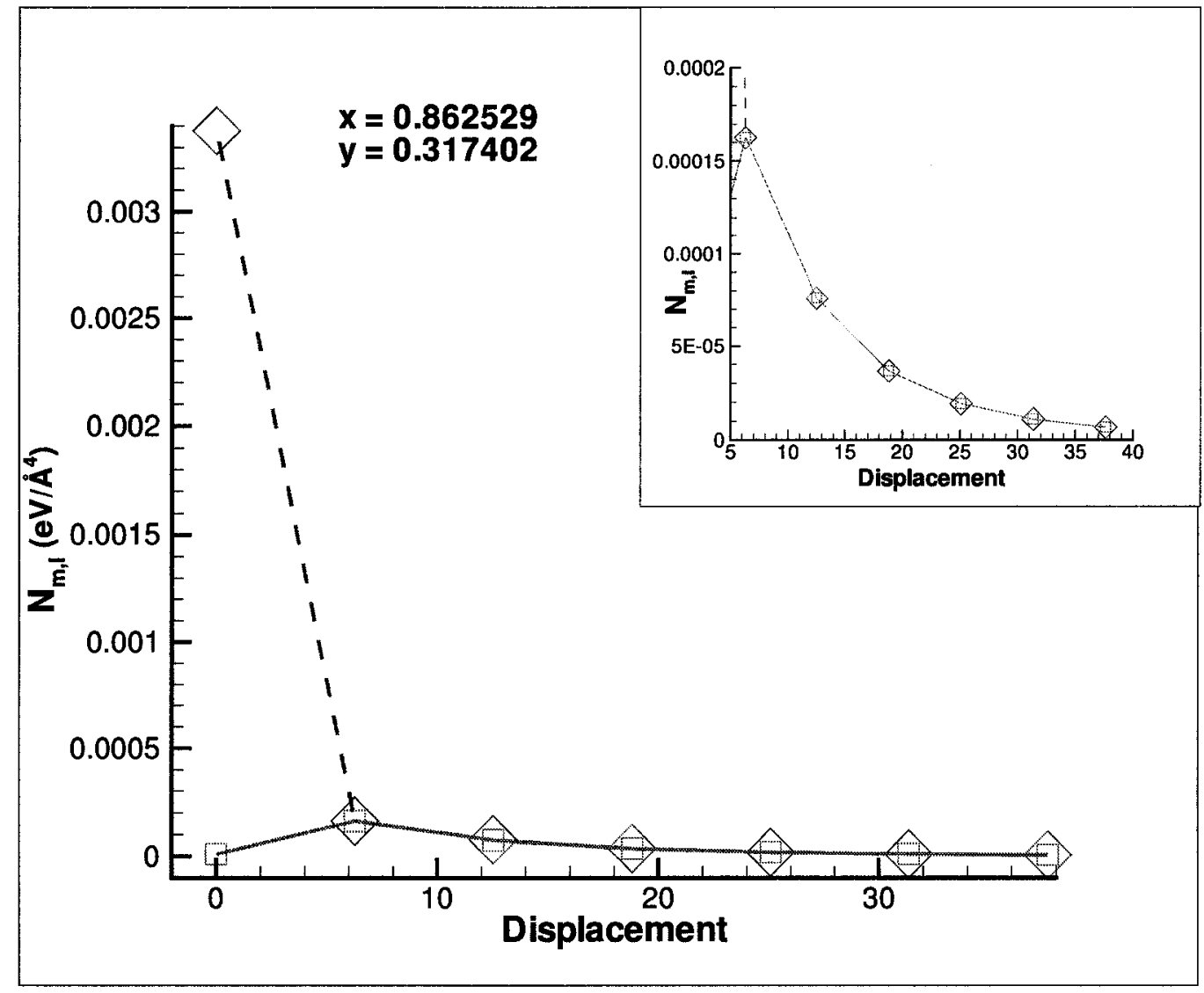

Figure 3.8: $\mathrm{N}_{m, l}$ versus depth curves for a column of atoms ( $x$ and $y$ coordinates exist within the range from 0.0 to 1.0 ) are shown to indicate the inaccuracy of $\mathrm{N}_{m, l}$ calculations near the surface. 


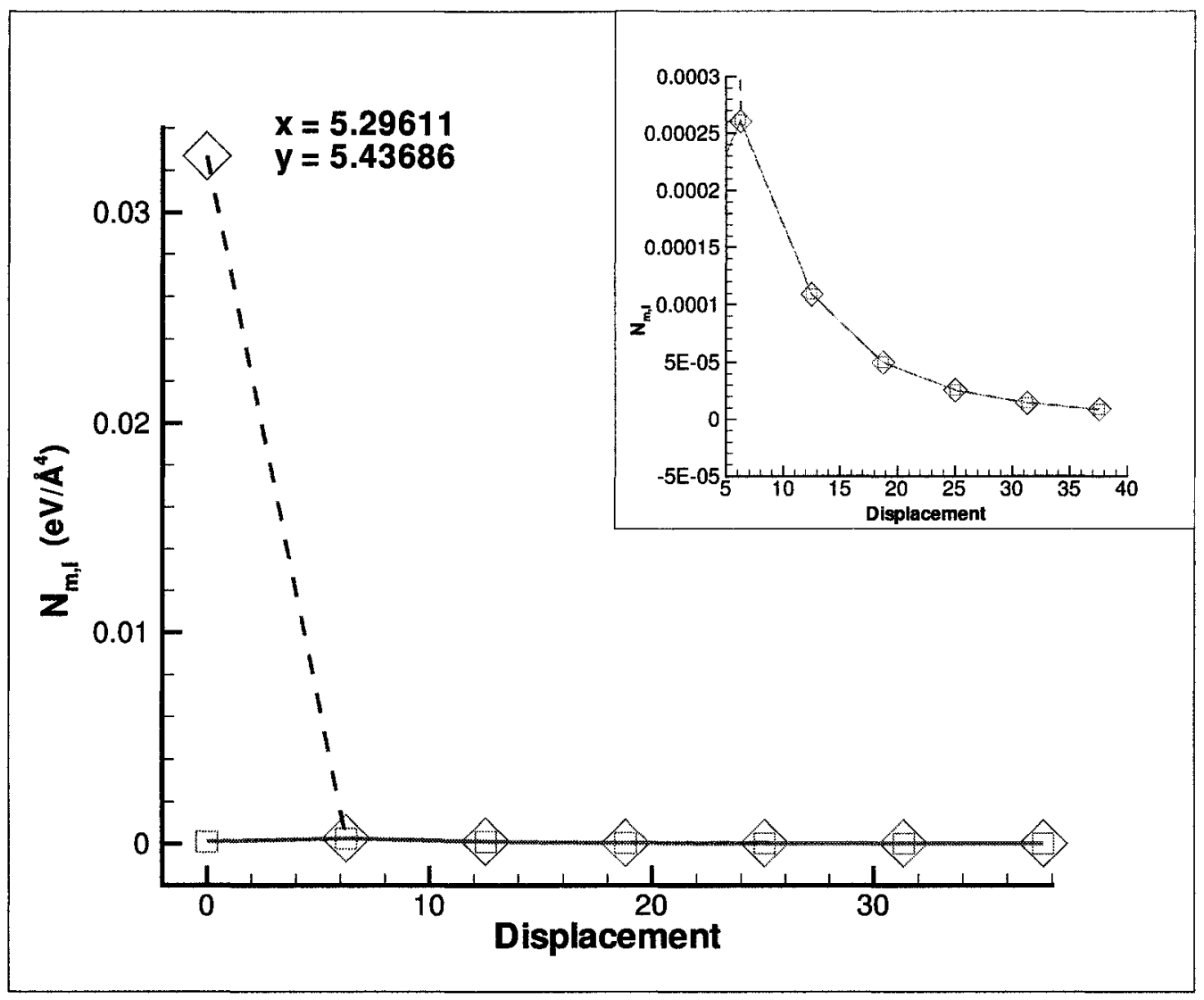

Figure 3.9: $\mathrm{N}_{m, l}$ versus depth curves for a column of atoms ( $\mathrm{x}$ and $\mathrm{y}$ coordinates exist within the range from 4.0 to 5.0 ) are shown to indicate the inaccuracy of $\mathrm{N}_{m, l}$ calculations near the surface. 


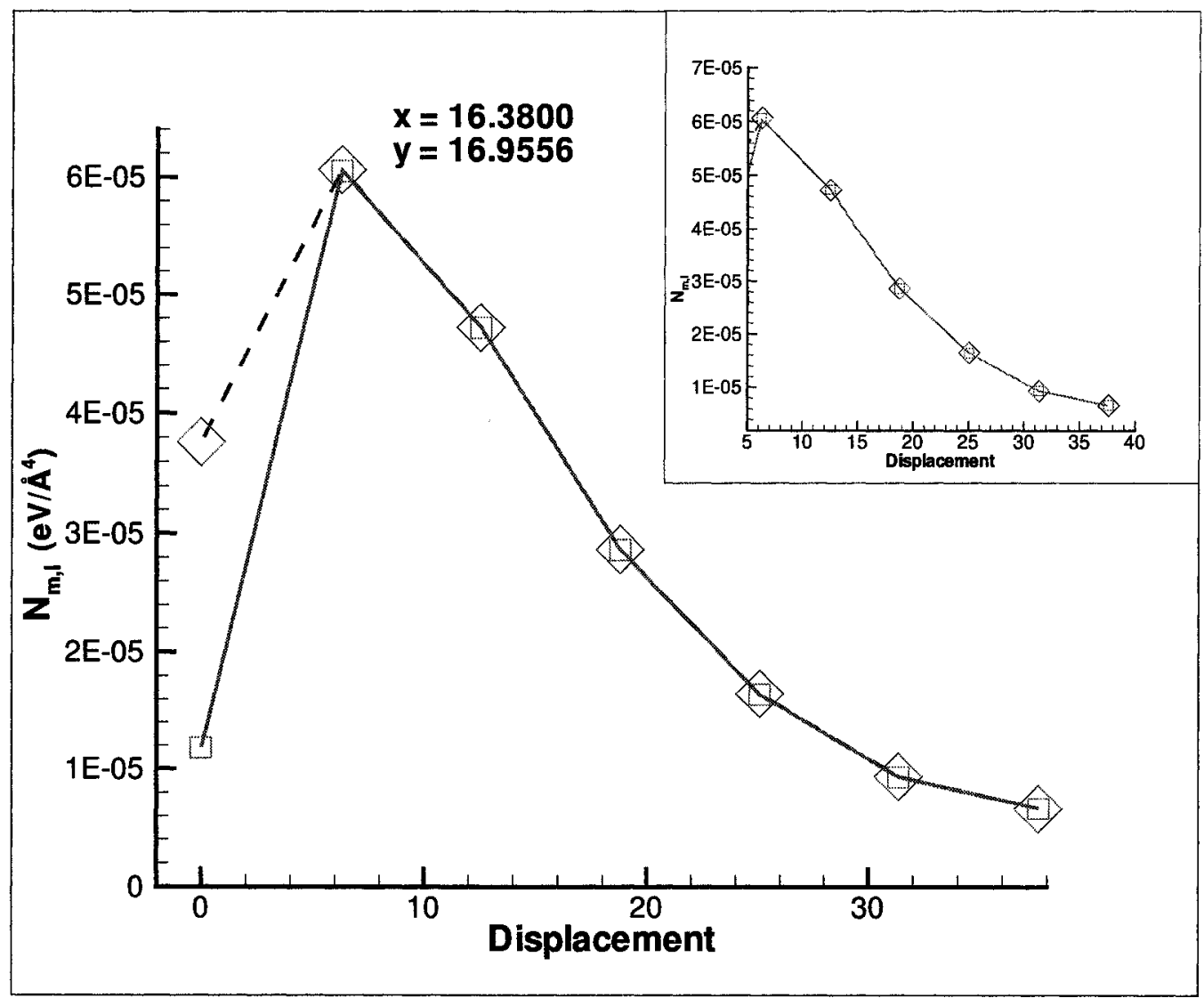

Figure 3.10: $\mathrm{N}_{m, l}$ versus depth curves for a column of atoms ( $\mathrm{x}$ and $\mathrm{y}$ coordinates exist within the range from 16.0 to 17.0 ) are shown to indicate the inaccuracy of $\mathrm{N}_{m, l}$ calculations near the surface. 


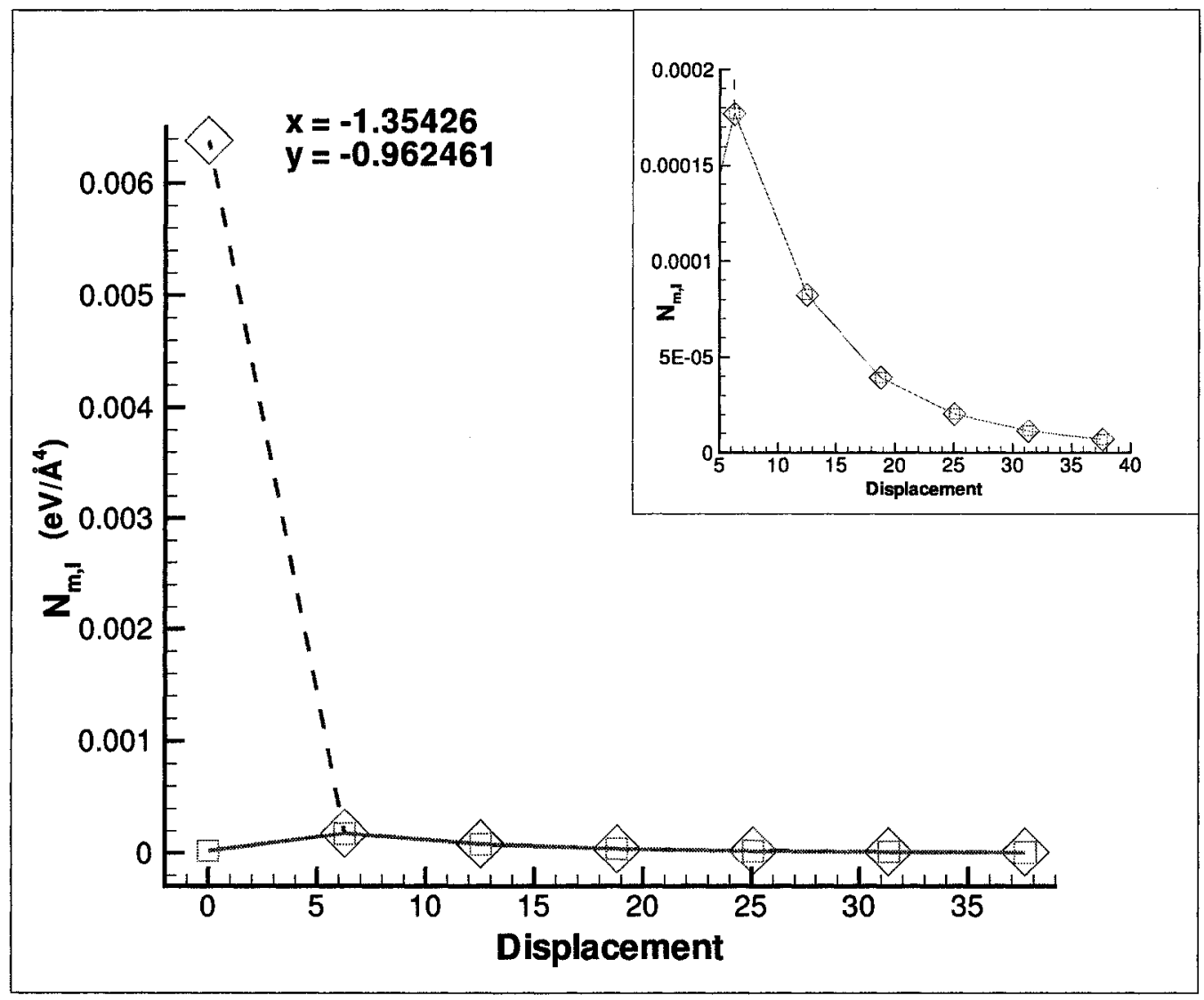

Figure 3.11: $\mathrm{N}_{m, l}$ versus depth curves for a column of atoms ( $\mathrm{x}$ and $\mathrm{y}$ coordinates exist within the range from -1.0 to 0.0 ) are shown to indicate the inaccuracy of $\mathrm{N}_{m, l}$ calculations near the surface. 


\subsubsection{Calculating $\mathbf{N}_{m, l}$}

A stress-gradient based criterion of dislocation nucleation, the "Acharya/Miller" criterion, is used to predict the mechanical conditions that lead to dislocation nucleation. The principal requirement of this criterion is that nucleation occurs when the maximum $\mathrm{N}_{m, l}$ is bigger than $\mathrm{N}_{c r i t}$ (the critical value of $\mathrm{N}_{m, l}$ which is also a material parameter) at some atom position. The calculation of $\mathrm{N}_{m, l}$ is given by:

$$
\mathrm{N}_{m, l}=|\mathbf{m} \cdot \operatorname{curl} \mathbf{T} \cdot \mathbf{l}|
$$

m : unit vector in the direction of the Burgers vector

$\mathbf{l}$ : unit line direction of the dislocation

curl $\mathbf{T}$ or $\nabla \times \mathbf{T}:$ the curl of the stress tensor, $\mathbf{T}$

Here, $\mathrm{N}_{m, l}$ is a scalar and used to determinate the event of dislocation nucleation. It requires us to interpolate the atomic level stresses [38] to compute the gradients at every atom in the crystal. This interpolation is performed using the "local optimal point interpolating"(LOPI) [37] method.

Figure 3.12 shows the visualization of a unit Burgers vector and unit line direction on a slip plane which is used to calculate $\mathrm{N}_{m, l}$ during the process of dislocation nucleation. In $3 \mathrm{D}$ crystal structure, there are a finite number of possible Burgers vectors (hence a finite number of values of $\mathbf{m}$ ) but dislocations will nucleate as a loop on the slip plane, and so all values of unit line direction 1 within the slip plane are possible. In order to calculate the maximum $\mathrm{N}_{m, l}$ value for the system, the stress gradient is obtained using LOPI method. Then, a number of discrete values of 1 (using 100 values in this work) around the loop are selected on a specific $\{111\}$ slip plane to calculate all the $\mathrm{N}_{m, l}$ values at each atom site. Finally, the maximum $\mathrm{N}_{m, l}$ value can be selected among all the atoms for an individual simulation depth, and the corresponding $\mathbf{l}$ and $\mathbf{m}$ can be recorded as the character of dislocation. 


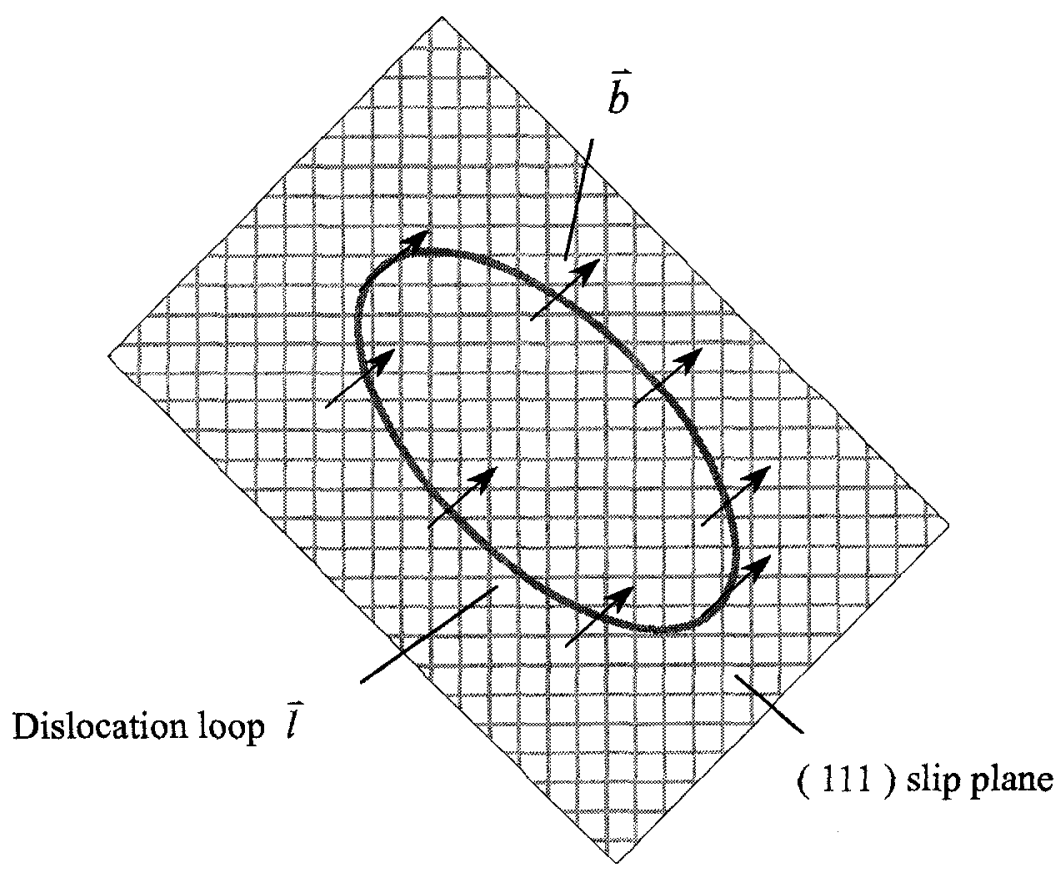

Figure 3.12: Distribution of Burgers vector $\vec{b}$ and line direction $\vec{l}$ along a nucleating dislocation loop on a (111) slip plane for fcc single crystal structure. 


\subsection{Method to determine actual defect nucleation}

The "Acharya/Miller" criterion [1] provides a method to predict the event of dislocation nucleation during plastic deformation, current work is to test the accuracy and efficiency of this method. During the analysis of real dislocations, the slip vector [15] must be used to provide quantitative information of deformation, and determine precisely where and when dislocations actually nucleate. This method will also tell us the dislocation type, allowing a direct test of the $\mathrm{N}_{m, l}$ criterion.

\subsubsection{Identifying the event of dislocation nucleation}

Figure 3.13 shows a method using the "Acharya/Miller" criterion to determine the actual defect nucleation. By identifying the events of dislocation nucleation, the indenter load drops suddenly during a nucleation event, at the same moment of the load drop, $\mathrm{N}_{m, l}$ at a specific atom point reaches to $\mathrm{N}_{c r i t}$, rapidly increasing after that moment. The phenomenon of load drop indicates the energy lost in the plastic deformation when dislocations begin to nucleate. $\mathrm{N}_{\text {crit }}$ can be easily found from the $\mathrm{N}_{m, l}$ versus displacement curve, it is the $\mathrm{N}_{m, l}$ value which is closely prior to the abrupt jump of $\mathrm{N}_{m, l}$ on the curve. Specially, the indenter load calculated within the MD package is the sum of the forces exerted on the indenter by its neighbor atoms inside the slab. The displacement refers to the motion of the indenter since the start of the simulation.

\subsubsection{The indication of Burgers vector: Slip vector}

In crystal materials (e.g., metals), plastic deformation occurs by the glide of dislocation on the corresponding slip planes. In order to make a more quantitative 


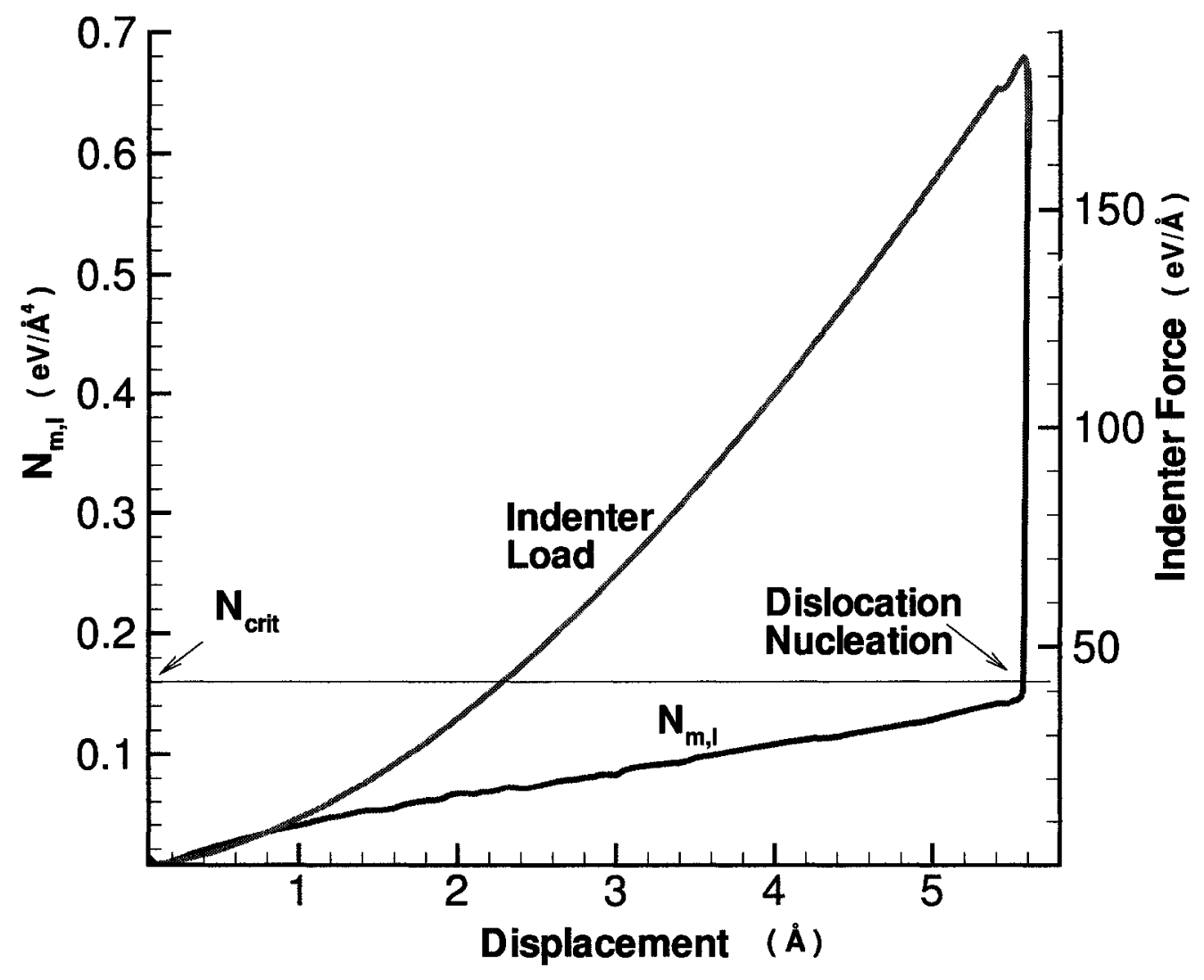

Figure 3.13: Dislocation Nucleation is evidenced by a abrupt drop of indenter load in the load-displacement and a simultaneous, sudden increase in the maximum $\mathrm{N}_{m, l}$. Show here for $\mathrm{Cu},[111]$ indentation direction. 
comparison between simulations and experiments, a quantity closely related to the Burgers vector can be defined for every atom in current simulation. This quantity is called the slip vector [15], defined as:

$$
s^{\alpha}=-\frac{1}{n_{s}} \sum_{\beta \neq \alpha}^{n}\left(x^{\alpha \beta}-X^{\alpha \beta}\right)
$$

In this expression, $n$ is the number of the nearest neighbors to atom $\alpha, n_{s}$ is the number of slipped neighbors, and $x^{\alpha \beta}$ and $X^{\alpha \beta}$ are the vector differences of atoms $\alpha$ and $\beta$ in the current and reference positions, respectively, the reference configuration is the arrangement of atomic positions associated with zero mechanical stress. This expression will result in the Burgers vector for the slip of adjacent atomic planes, where the atom lies on one of those planes.

Figure 3.14 schematically indicates the derivation of the slip vector, it will have a large magnitude for any inhomogeneous deformation near an atom and will provide quantitative information about the deformation. The spatial distribution of the slip vectors modulus $\left|s^{\alpha}\right|$ around the nano-indentation result, with a suitable color scale, is shown in the Figure 3.15. Note that only atoms with large magnitude of the slip vector are made visible here, moreover, the components of slip vector help to identify the character of dislocations. In current work, the slip vector is used to analyze the dislocation structures produced during nano-indentation.
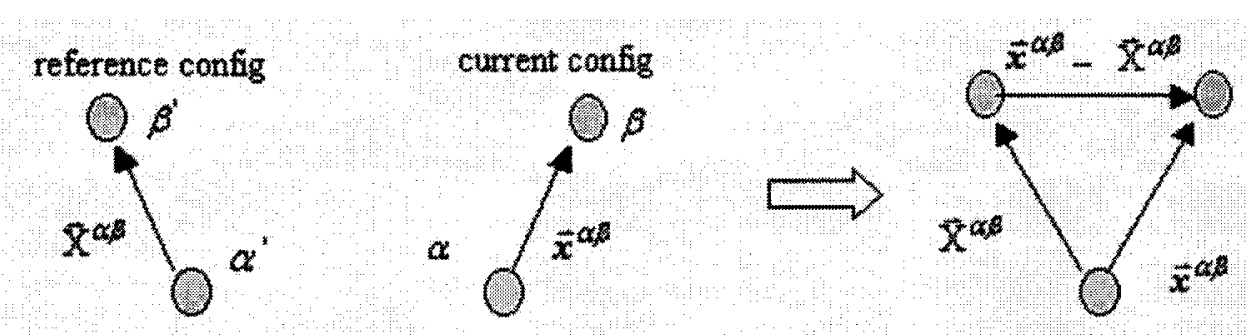

Figure 3.14: the vector description of the slip vector 


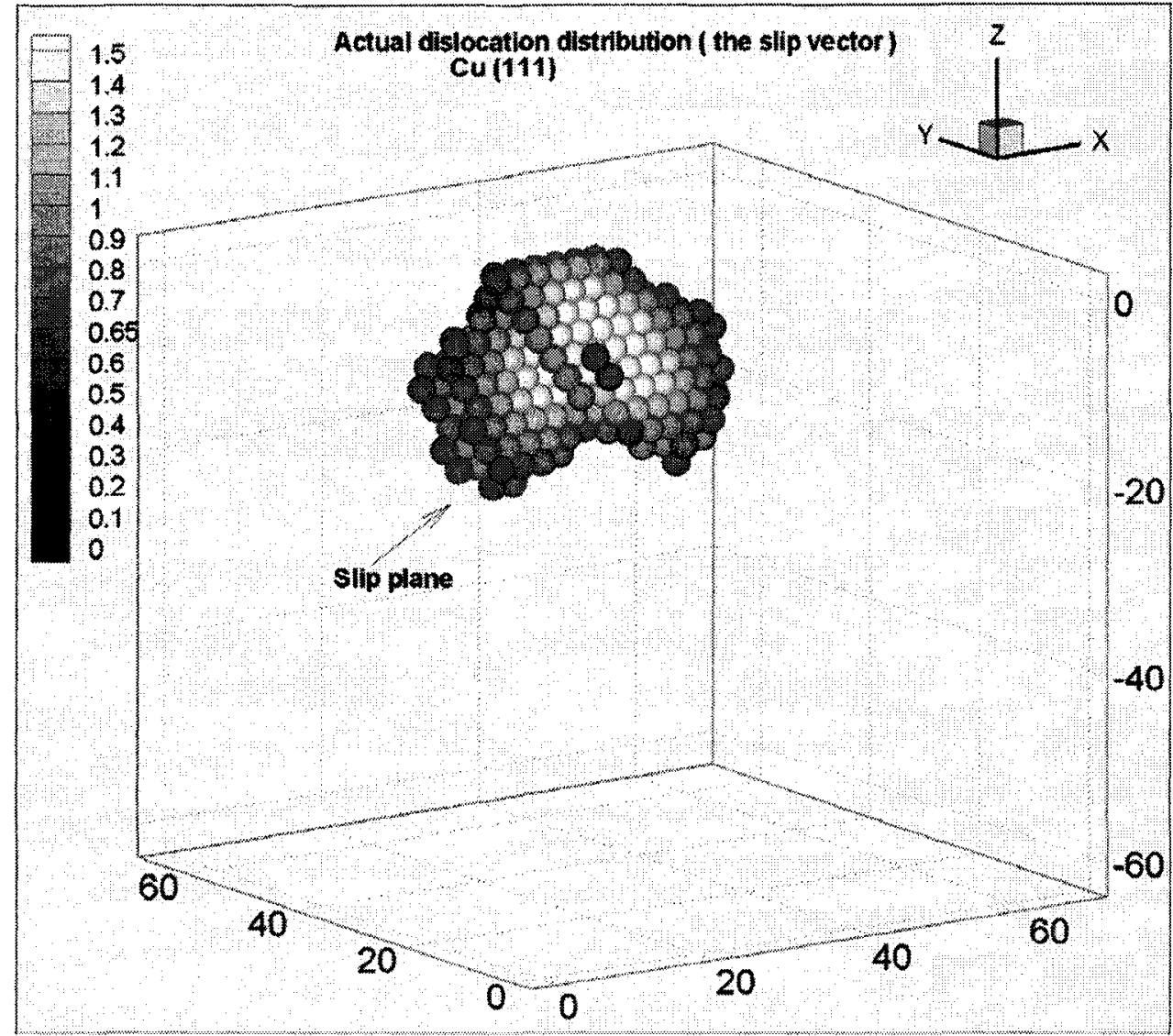

Figure 3.15: The spatial distribution of slip vector in MD simulation 


\section{Chapter 4}

\section{Results and Discussion}

\subsection{Pictorial representation of results}

Pictorial views of dislocations make it possible to analyze the results of dislocation nucleation obtained from the simulations described in chapter 3 . According to the "Acharya/Miller" criterion, the event of dislocation nucleation can be predicted by the abrupt jump of the maximum $\mathrm{N}_{m, l}$ value during the process of indenter penetration. The type and character of dislocations at nucleation are determined by studying the visualized distribution of slip vector inside the single crystal structure, and the process of nucleation is dynamically recorded during MD simulation. By comparing the visualized distribution of $\mathrm{N}_{m, l}$ and slip vector, the efficiency and accuracy of the method used to predict the location and moment of nucleation is tested.

For brevity, the $\mathrm{Cu}(001), \mathrm{Ni}(001), \mathrm{Cu}(110), \mathrm{Ni}(110), \mathrm{Cu}$ (111) and $\mathrm{Ni}$ (111) model are refered to present various indentation tests in the following sections of this chapter. For all these simulations, a spherical indenter with a radius of 60 angstroms is put to the indentation surface of the slab, then, a displacement controlled nanoindentation is performed with a speed of $0.05 \AA / p$ sec. This results in a series of continuously varying dislocation structures because of the homogeneous dislocation nucleation and glide on the $\{111\}$ close-packed slip planes. 
Figure 4.1 shows the method used to identify the type of slip planes, the plane equation is $a x+b y+c z+d=0$ is assumed and three points are selected on each dislocation loop (as shown, for example, in figure 3.15), then separately substitute their coordinates into the plane equation to create a group of equations. Ultimately, this equation group is solved to obtain the parameters of each plane(i.e., $a, b, c, d)$ which will be used to calculate the indices of lattice planes. On the other hand, to determine the corresponding crystal plane, the method of coordinate transformation described in Appendix $B$ is used in current dynamo code.

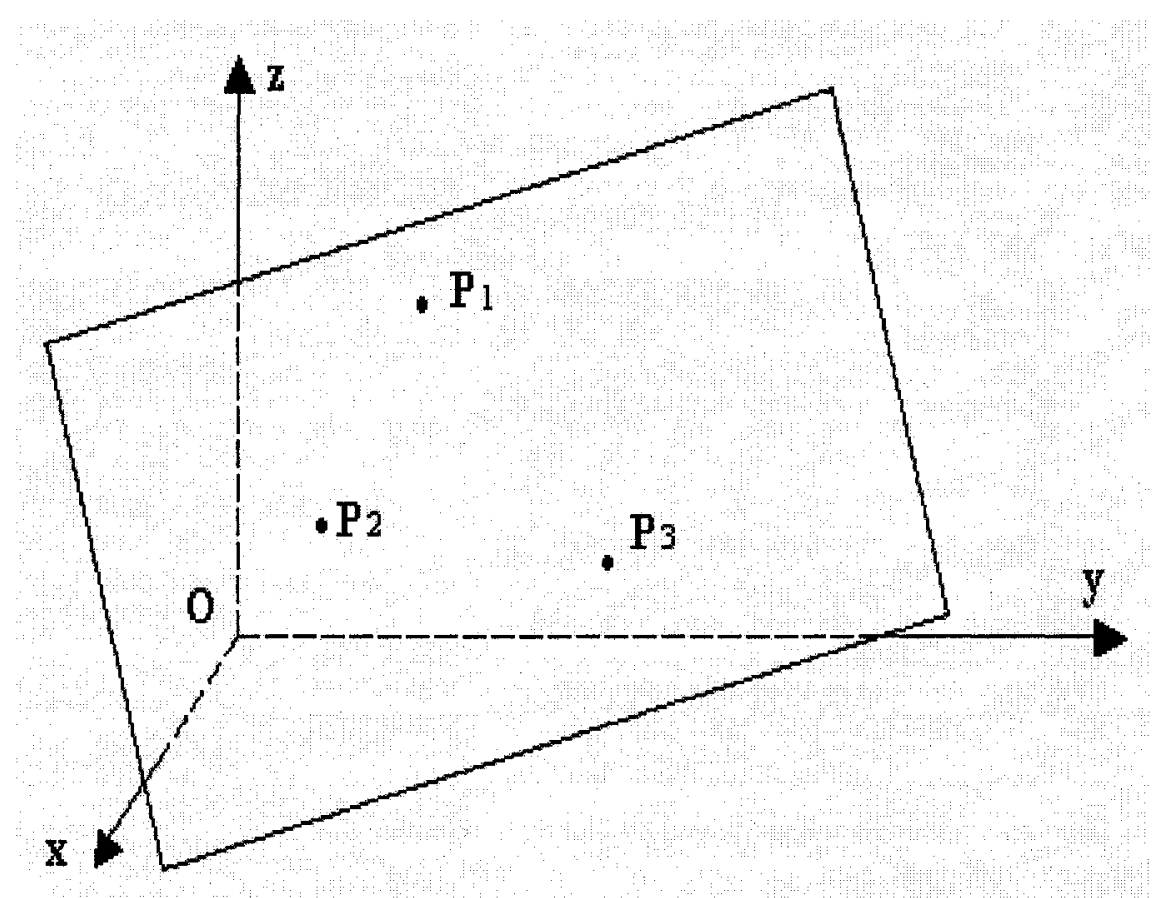

Figure 4.1: Calculating the indices of a lattice plane with three points on the plane.

\subsection{1 $\mathrm{Cu}(001)$ model}

In order to study the nature of plastic deformation and characterize the dislocation core structure nucleated, the deformed regions are visualized by coloring the atoms 
identified with the slip vector. Figure 4.2 to Figure 4.5 show a few snapshots of the deformed regions at various stages of dislocation nucleation near the first plastic yield point for $\mathrm{Cu}(001)$ model to illustrate the evolution of the dislocation structure.

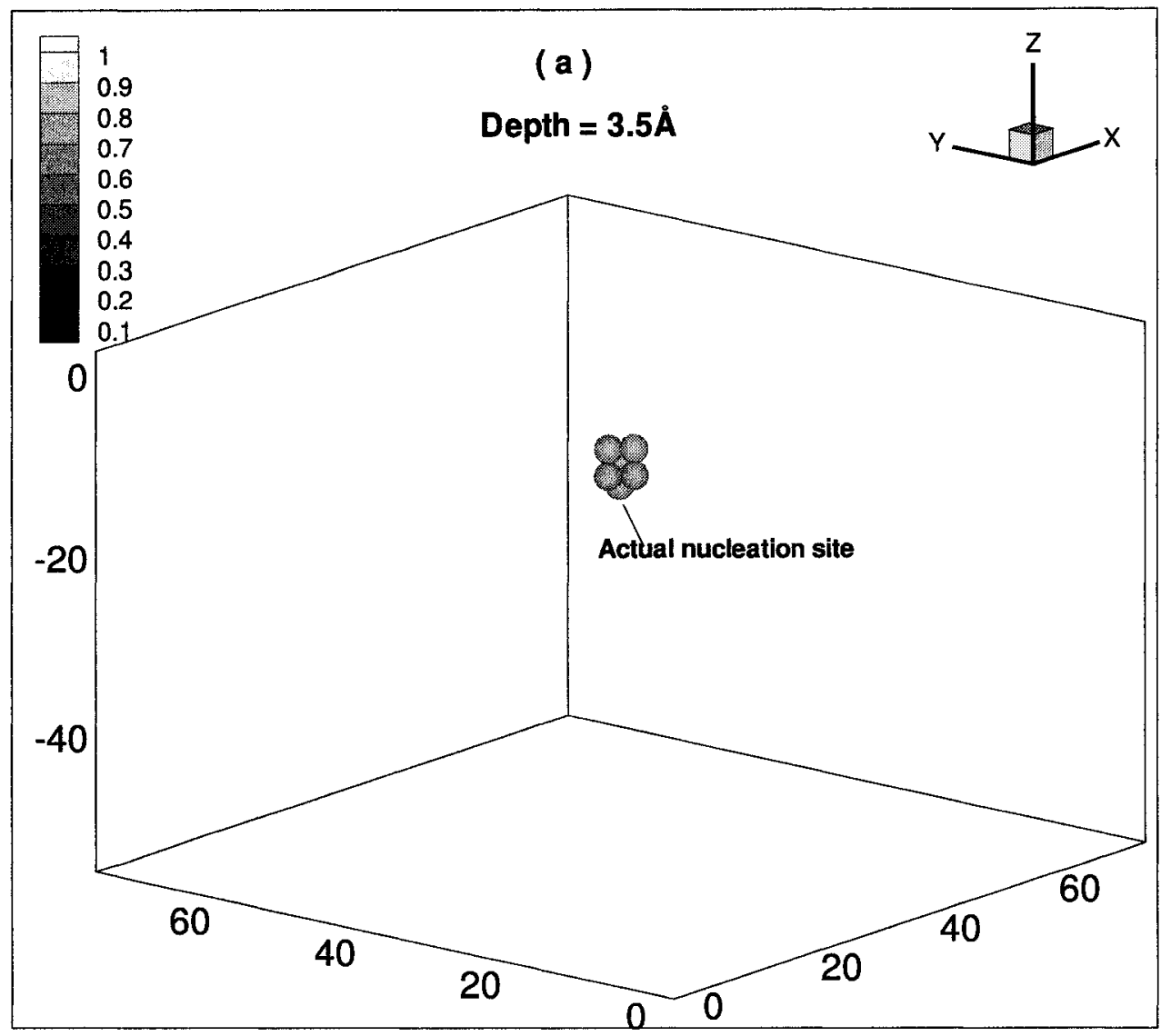

Figure 4.2: The process of dislocation nucleation is illustrated by the variation of actual dislocations (presented by the slip vector) for the $\mathrm{Cu}(001)$ model. Snapshot (a): Dislocations begin to nucleate below the top indentation (001) surface inside the indenter contact area. 


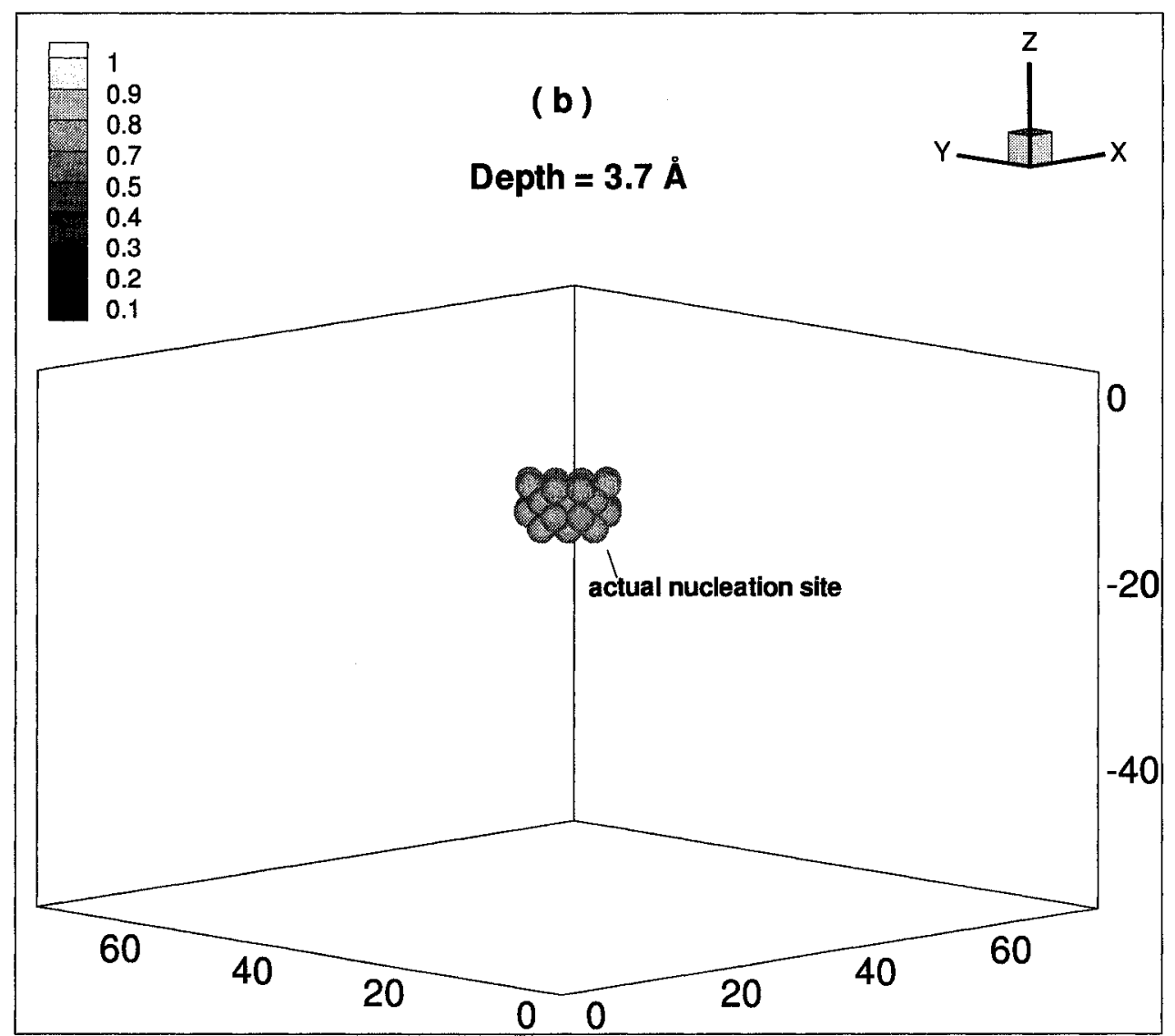

Figure 4.3: Snapshot (b): These dislocations grow in size. 


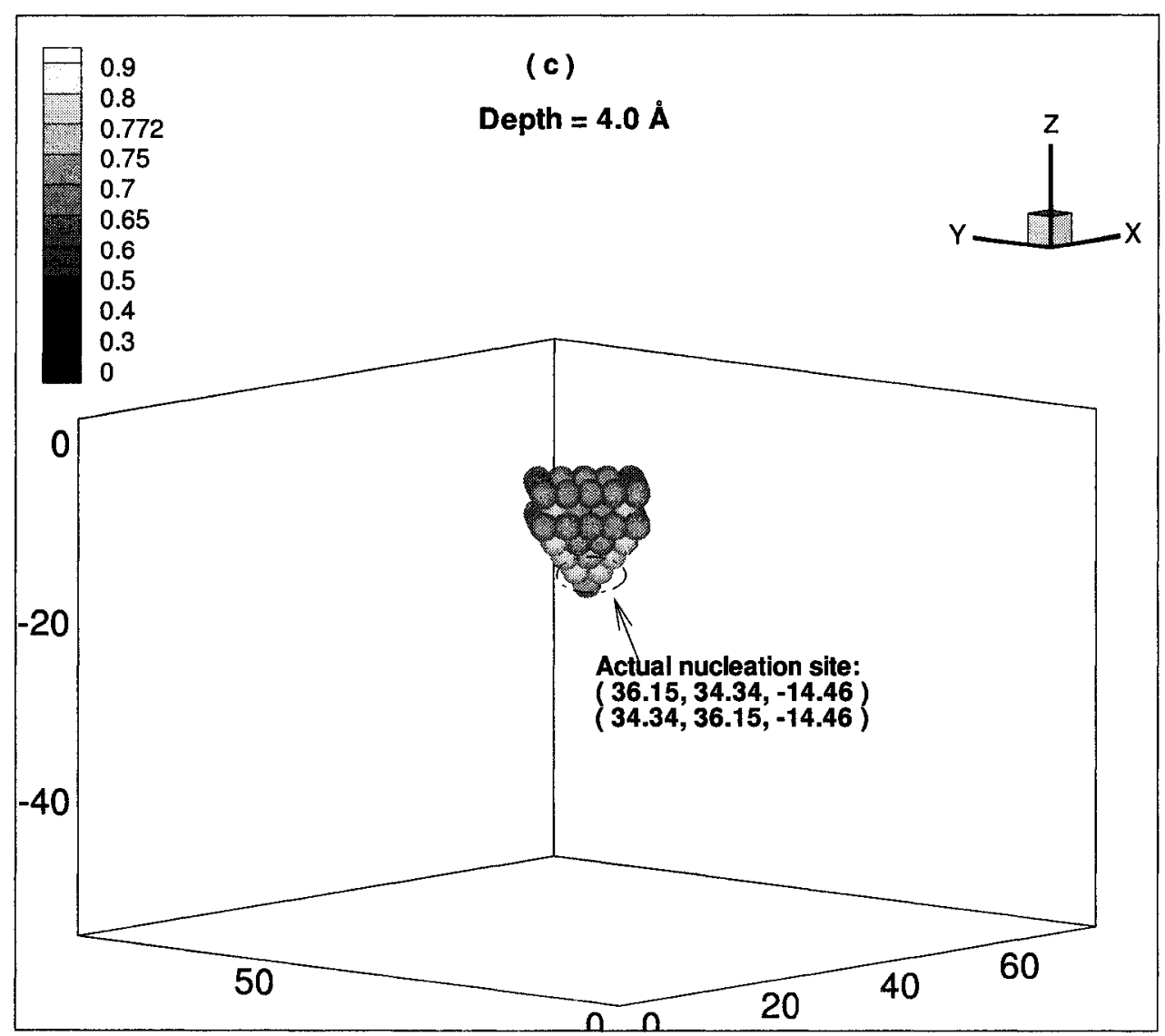

Figure 4.4: Snapshot(c): These dislocation loops intersect with the loops on the adjacent planes forming a pyramidal nucleation structure, the actual nucleation site is on the bottom tip of the pyramidal structure. 


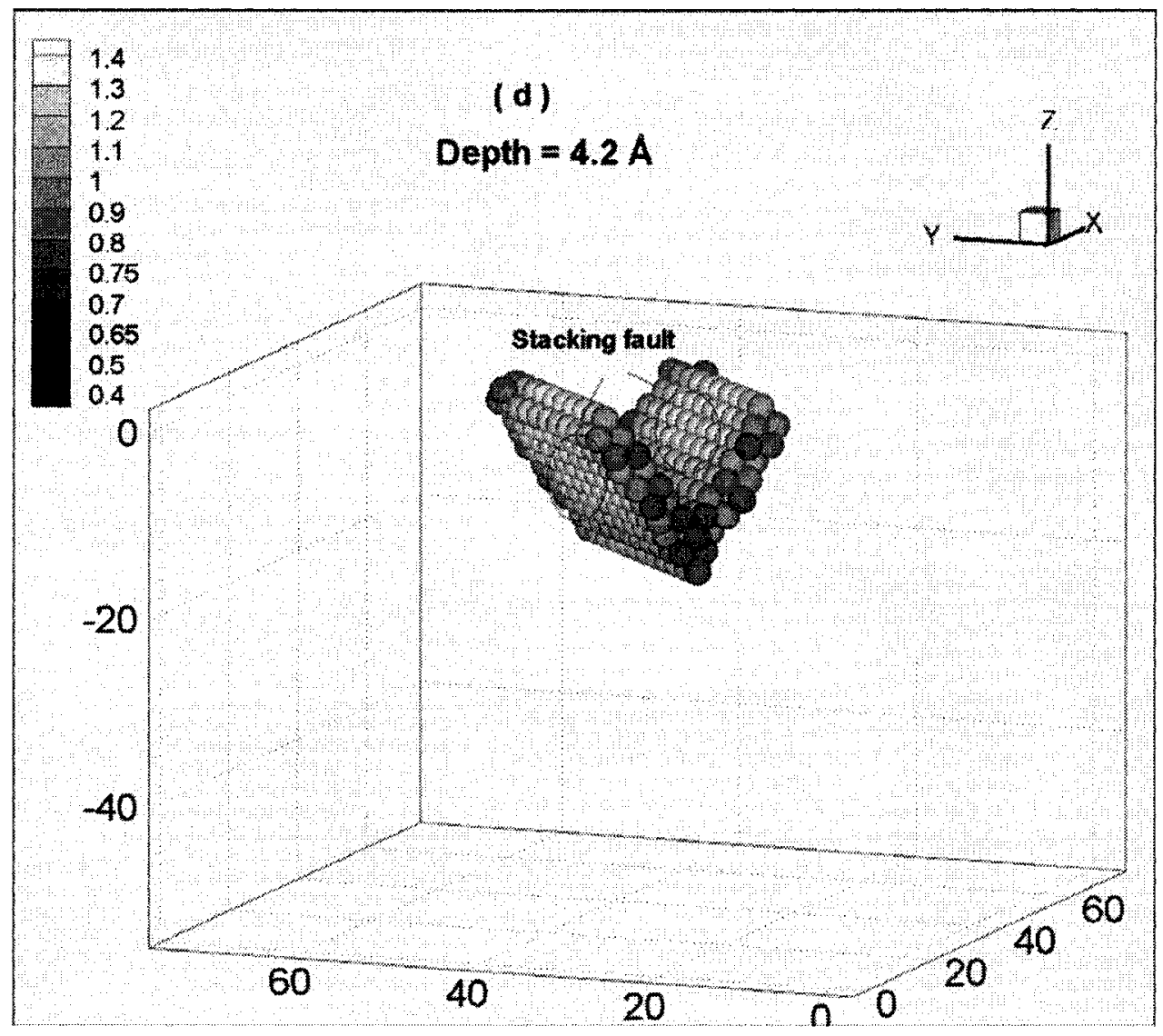

Figure 4.5: Snapshot (d): Dislocation loops prefer to move on two opposite $\{111\}$ slip planes and form a V-shaped defect structure, the magnitude of slip vector increases to the length of Shockley partial dislocation. 
Figure 4.2 shows the locations of nucleation are below the top indentation (001) surface inside the contact area, then the core of dislocation grows in size to form an extended structure shown in the Figure 4.3. Next, the nucleation of partial dislocation loops occurs on four $\{111\}$ slip planes. After nucleation, these dislocation loops move into the solid and intersect with the loops on the adjacent planes forming a pyramidal dislocation structure shown in the Figure 4.4. Afterwards, the Figure 4.5 illustrates that dislocation loops prefer to move on two opposite $\{111\}$ planes and form a V-shaped defect structure.

In the next a few paragraphs, the methods used to determine the character of these defects are proved. The magnitude of the slip vector is found to close $1.66 \AA$ for $\mathrm{Ni}$ and $1.47 \AA$ for $\mathrm{Cu}$, and the directions of the slip vector are consistent with specific $\langle 112\rangle$ partial dislocations on $\{111\}$ planes in fcc metals. Therefore, the dislocation nucleated on the $(1 \overline{1} \overline{1})$ plane is the $\frac{1}{6}[\overline{1} 1 \overline{2}]$ Shockley partial dislocation, similarly, the $\frac{1}{6}[\overline{1} 12]$ shockley partial dislocation is nucleated on the (111) plane. The defects consist of intersecting intrinsic stacking faults on the two $\{111\}$ planes which intersect the top indentation (001) surface with a fourfold symmetry. This V-shaped dislocation structure is in excellent agree with the permanent deformation structures observed in experiment [13],[41],[4].

Figure 4.6 shows the visualized contour of the slip vector after the moment of dislocation nucleation when the displacement of indenter is equal to $4.2 \AA$. It is clear that the slip vectors become significant on two opposite $\{111\}$ slip planes along $\langle 112\rangle$ directions to form a V-shaped defect structure. The left plane is found to be the

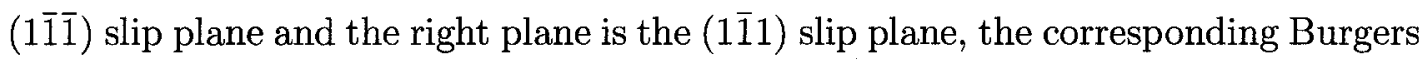
vectors are $\frac{1}{6}[\overline{1} 1 \overline{2}]$ and $\frac{1}{6}[\overline{1} 12]$. 


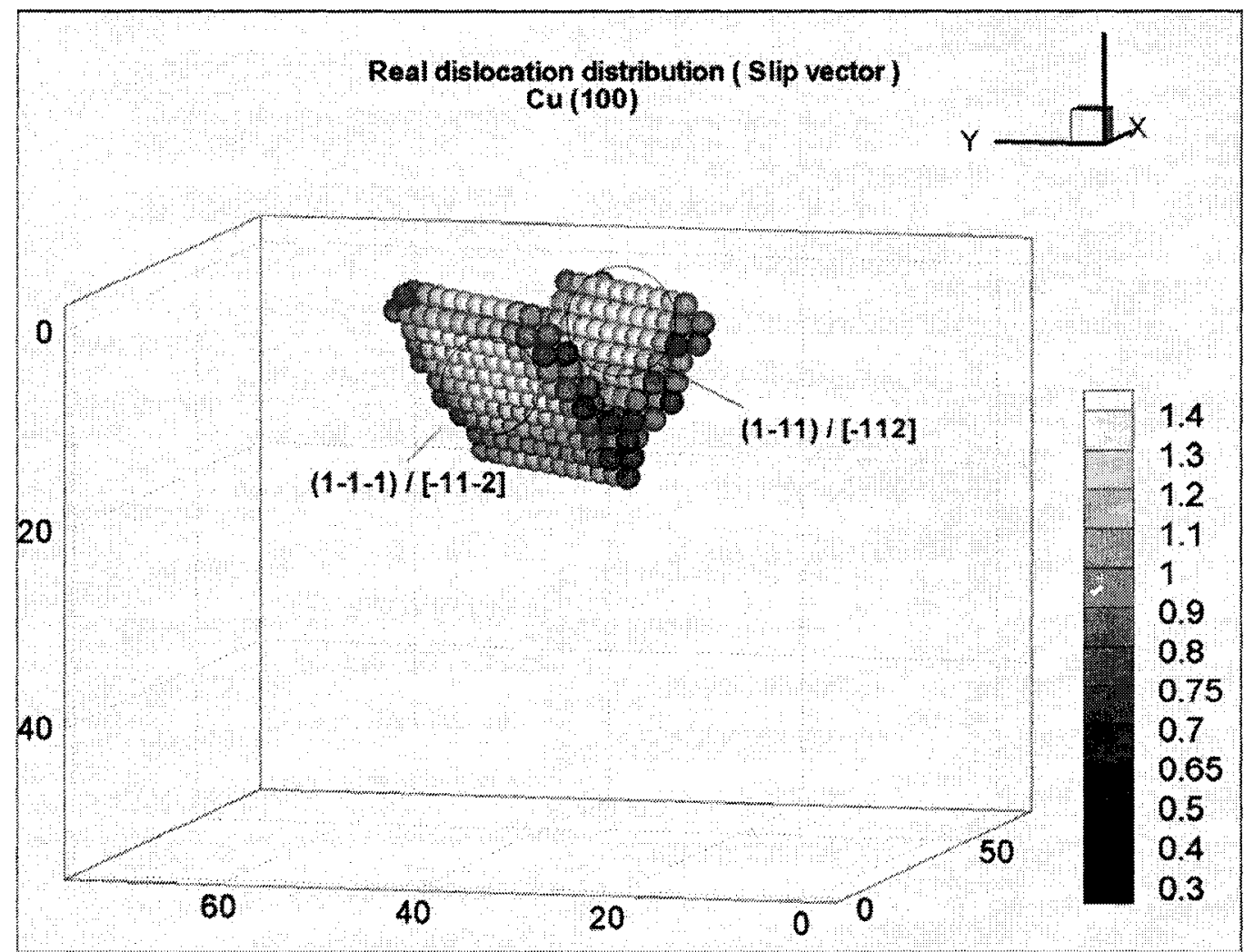

Figure 4.6: Contour of slip vectors on slip planes to demonstrate the actual dislocation structure beneath the indentation (001) surface. 


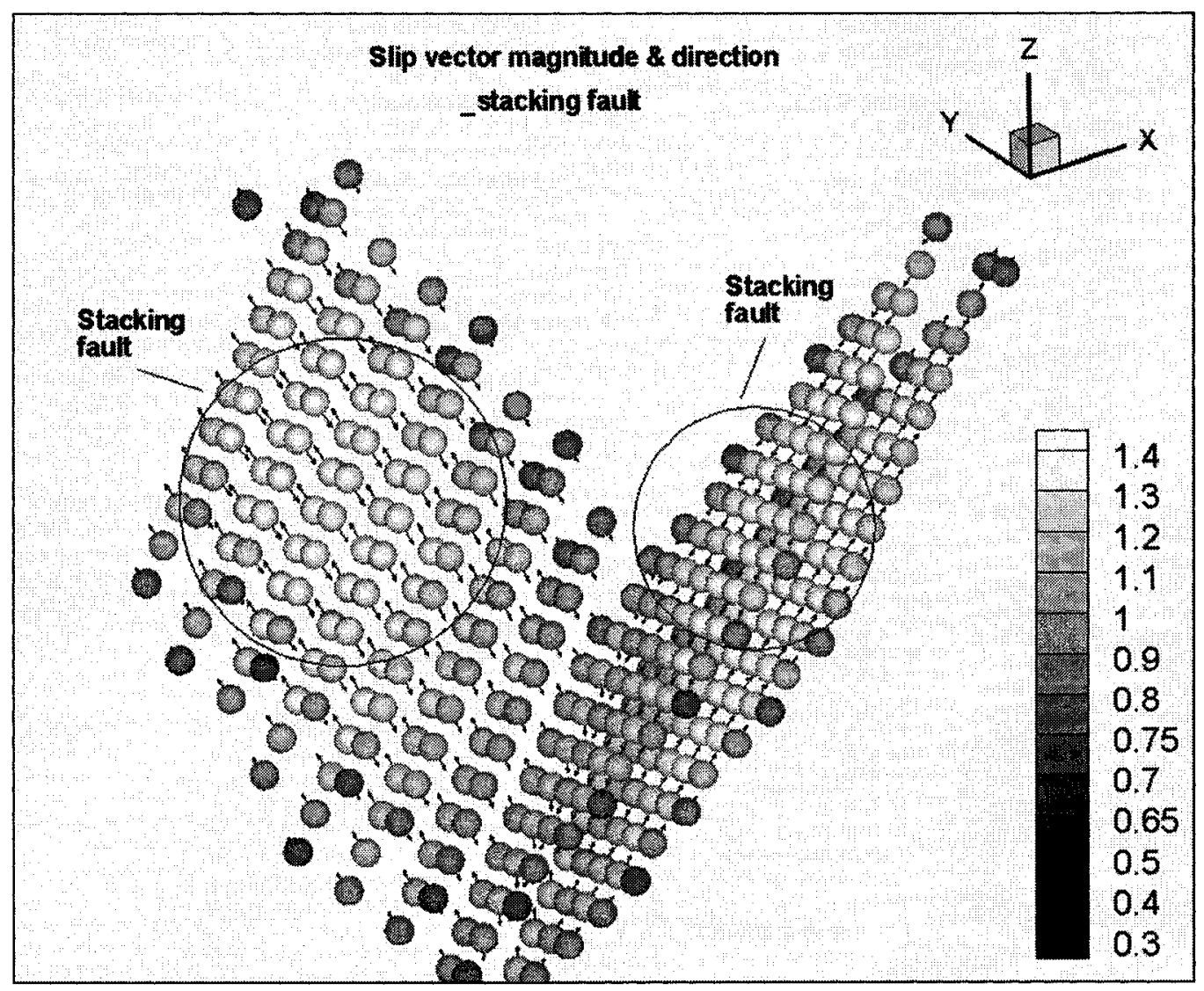

Figure 4.7: Vector graph of slip vectors on slip planes to demonstrate the magnitude and direction of Burgers vector for $\mathrm{Cu}(001)$ model.

Figure 4.7 shows the vector graph of slip vectors bounding stacking faults at the same moment as Figure 4.6. The maximum magnitude of the slip vector (around $1.47 \AA$ for $\mathrm{Cu}$ ) and its components prove that the dislocations after nucleation are Shockley partial dislocations. 


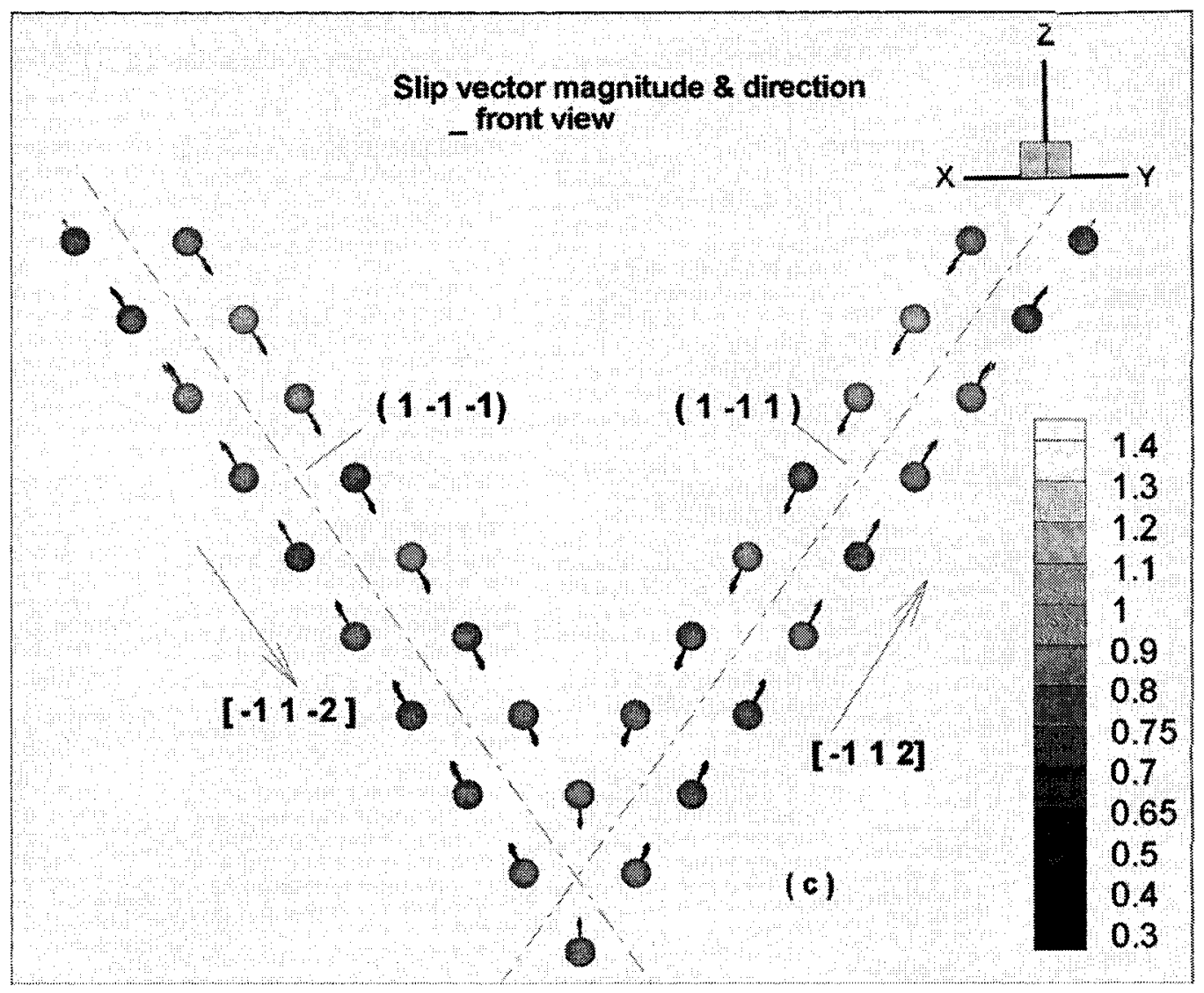

Figure 4.8: Front view of the actual dislocation structure to demonstrate the magnitude and direction of Burgers vector for $\mathrm{Cu}(001)$ model. 
Figure 4.8 shows the front view of dislocation structure at the same indentation moment as Figure 4.6 and Figure 4.7. It is clear that two layers of atoms with large magnitude of slip vector on each slip plane are shown, the slip vector on the two layers have the same magnitude of slip vector along the opposite slip direction. It is also obvious that the maximum magnitude of slip vector always happens inside the stacking fault with Shockley partial dislocations.

The goal of the "Acharya/Miller" criterion is to be able to predict a dislocation nucleation event by monitoring the variation of the maximum $\mathrm{N}_{m, l}$ versus displacement curve during nano-indentation simulation (i.e., an abrupt jump on the curve is related to a nucleation event). Therefore, such character of discontinuity of $\mathrm{N}_{m, l}$ versus displacement curve can be used to identify dislocation nucleation events, one of current goals is to prove the accuracy and efficiency of this method.

To examine the capability of the "Acharya/Miller" criterion to predict dislocation nucleation events, Figure 4.9 shows the correlation of two curves: the indenter load versus displacement curve and the maximum $\mathrm{N}_{m, l}$ versus displacement curve. The maximum $\mathrm{N}_{m, l}$ versus displacement curve is shown as the bottom black curve, the indenter load versus displacement curve is represented as the top light curve.

Initially, the indenter load versus displacement curve displays elastic behavior until the load drops abruptly at the first yield point. Such a phenomenon indicates the onset of initial plasticity and is related to the nucleation event to partially release the energy below the contact region. It is also seen that the $\mathrm{N}_{m, l}$ value gradually increases with the displacement of indenter during elastic deformation, whereas, at the same moment of the drop of indenter load, the $\mathrm{N}_{m, l}$ value suddenly jumps to a large value and this event is related to the beginning of dislocation nucleation. The dislocation nucleation events can be characterized by the drop of indenter load. At the same moment of the drop, the $\mathrm{N}_{m, l}$ value also suddenly increases from a 
critical value $\mathrm{N}_{\text {crit }}$ (a specific material property) to a large value. In other words, dislocations begin to nucleate when the $\mathrm{N}_{m, l}$ value reaches to $\mathrm{N}_{\text {crit }}$. Therefore, the $\mathrm{N}_{m, l}$ value can be used to predict the dislocation nucleation events by monitoring the variety of the variable $\mathrm{N}_{m, l}$.

The bottom part of Figure 4.9 is the enlarged view of the top part, the correlation of these two curves can be observed clearly, and nucleation happens at indenter depth $4.05 \AA$ for $\mathrm{Cu}(001)$ model. Specifically, $\mathrm{N}_{\text {crit }}$ of $\mathrm{Cu}$ is $0.16 \pm 0.02\left(\mathrm{eV} / \AA^{4}\right)$ in this simulation. Observing the abrupt jump of $\mathrm{N}_{m, l}$ with displacement is proved to be an efficient method to identify the event of dislocation nucleation.

Figure 4.10 shows the visualization of the actual dislocation nucleation presented by the slip vector and as predicted by the $\mathrm{N}_{m, l}$ value in fcc single crystalline metal. Comparing the actual site of dislocation nucleation and the predicted site using the criterion, the site of actual nucleation and the character of slip system (slip plane and Burgers vector) are proved to be correctly predicted in advance.

The top part of Figure 4.10 is two snapshots of the distribution of $\mathrm{N}_{m, l}$ value inside the $\mathrm{Cu}(001)$ crystal slab at a moment just before the nucleation event, atom color indicates the magnitude of $\mathrm{N}_{m, l}$ and only the atoms whose $\mathrm{N}_{m, l}$ is within the maximum $\mathrm{N}_{m, l}$ range are shown. The maximum value of $\mathrm{N}_{m, l}$ always occurs together with the Shockley partial dislocations $(1 \overline{1} \overline{1}) / \frac{1}{6}[\overline{1} 1 \overline{2}]$ and $(1 \overline{1} 1) / \frac{1}{6}[\overline{1} 12]$ inside the dislocation loops when the indenter displacement is around $4.0 \AA$, which is prior to the nucleation event. To clearly show the character of the slip system, the right-top snapshot of Figure 4.10 shows an enlarged view of the left-top one. According to the "Acharya/Miller" criterion of nucleation, the dislocations are predicted to happen as these two kinds of Shockley partial dislocations, and at the location indicated. 

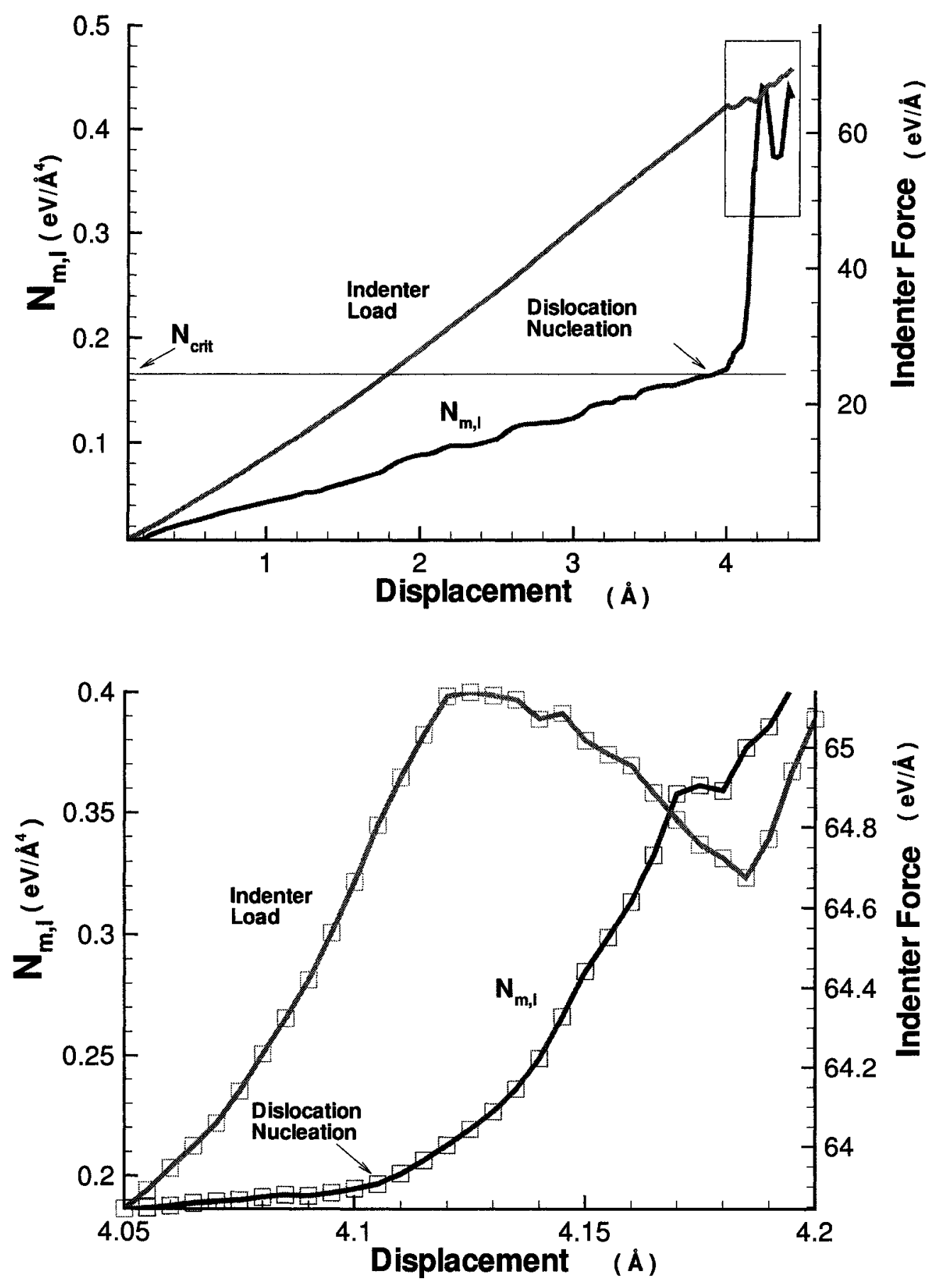

Figure 4.9: The correlation of two curves: the indenter load versus displacement curve and the maximum $\mathrm{N}_{m, l}$ versus displacement curves for $\mathrm{Cu}$ (001) model. The lower figure is an enlargement of the late stages of the top graphs. 
The bottom part of Figure 4.10 is another two snapshots of actual dislocation distribution presented by slip vector at a moment immediately after nucleation. The atom color indicates the magnitude of slip vector, and the visible atoms have larger magnitude of slip vector than the hidden atoms inside the slab. Specifically, it is a visualization of the precise atomic structure of the dislocations underneath the indentation surface after the nucleation event at the indenter depth $4.4 \AA$. The components of slip vector indicate that dislocations have moved along $\langle 112\rangle$ directions on two $\{111\}$ slip planes as Shockley partial dislocations and then form a V-shaped half-loop dislocation structure. Observing the bottom snapshots, two black circles

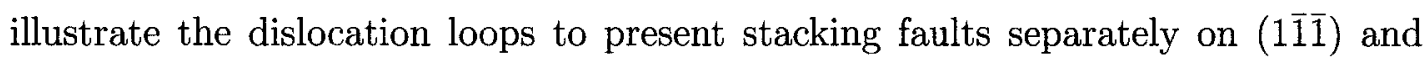
(111) slip planes with Shockley partial dislocations $\frac{1}{6}[\overline{1} 1 \overline{2}]$ and $\frac{1}{6}[\overline{1} 12]$. The intersection of the two planes defines a line that parallels to the top indentation (001) surface. Another fact used to determine that these are Shockley partial dislocations is that the maximum magnitude of slip vector is around $1.47 \AA$ which is close to the value of Shockley partial dislocation in $\mathrm{Cu}$. Similarly, the right-bottom snapshot in Figure 4.10 is an enlarged and rotated view of the left-bottom snapshot used to clearly show the configurations of the slip system.

Comparing the top part and bottom part of Figure 4.10, an exciting finding is that the dislocations indicated by the slip vector analysis are as the same Shockley partial dislocations as the results predicted using the maximum $N_{m, l}$ value. Therefore, the fact is verified that the slip systems decided by the maximum $\mathrm{N}_{m, l}$ value according to the "Acharya/Miller" criterion before nucleation agree with the actual slip systems determined by the slip vector analysis after the event of dislocation nucleation. It indicates that dislocation nucleation can be accurately predicted with this criterion. 

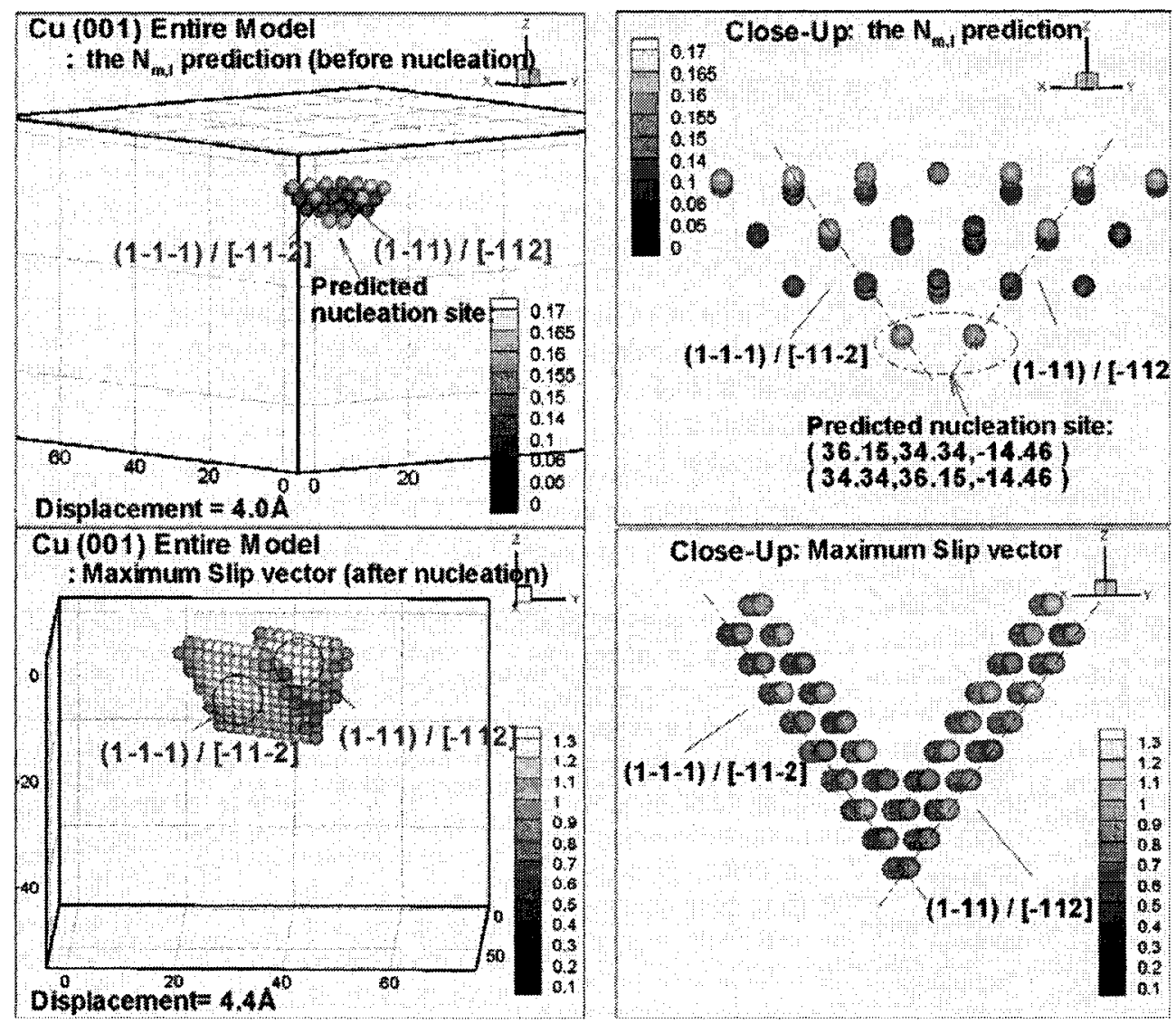

Figure 4.10: The comparison of the $\mathrm{N}_{m, l}$ prediction and actual dislocations is shown for the $\mathrm{Cu}(001)$ model. The bottom part of the figure is a figure of the slip vector distribution immediately after the nucleation event, and the top part of the figure is the maximum $\mathrm{N}_{m, l}$ value distribution throughout the atom slab. 


\begin{tabular}{|c|c|c|c|c|c|}
\hline \multirow{2}{*}{ Depth } & \multirow{2}{*}{$\operatorname{Maxi} \mathrm{N}_{m, l}$} & \multicolumn{4}{|c|}{ Predicted nucleation site and dislocation character } \\
\hline & & Coor_x & Coor_y & Coor_z & Slip system \\
\hline \multirow[t]{2}{*}{4.05} & 0.1865388 & 36.1499998 & 34.3424998 & -14.459999 & $(1 \overline{1} 1) /[\overline{1} 12]$ \\
\hline & 0.1865388 & 34.3424998 & 36.1499998 & -14.459999 & $(1 \overline{1} \overline{1}) /[\overline{1} 1 \overline{2}]$ \\
\hline \multirow[t]{2}{*}{4.055} & 0.1870113 & 36.1499998 & 34.3424998 & -14.459999 & $(1 \overline{1} 1) /[\overline{1} 12]$ \\
\hline & 0.1870113 & 34.3424998 & 36.1499998 & -14.459999 & $(1 \overline{1} \overline{1}) /[\overline{1} 1 \overline{2}]$ \\
\hline \multirow[t]{2}{*}{4.06} & $0.187717 \overline{7}$ & 36.1499998 & 34.3424998 & -14.459999 & $(1 \overline{1} 1) /[\overline{1} 12]$ \\
\hline & 0.1877177 & 34.3424998 & 36.1499998 & -14.459999 & $(1 \overline{1} \overline{1}) /[\overline{1} 1 \overline{2}]$ \\
\hline \multirow[t]{2}{*}{4.065} & 0.1889784 & 36.1499998 & 34.3424998 & -14.459999 & $(1 \overline{1} 1) /[\overline{1} 12]$ \\
\hline & 0.1889784 & 34.3424998 & 36.1499998 & -14.459999 & $(1 \overline{1} \overline{1}) /[\overline{1} 1 \overline{2}]$ \\
\hline \multirow[t]{2}{*}{4.07} & 0.1895311 & 36.1499998 & 34.3424998 & -14.459999 & $(111) /[112]$ \\
\hline & 0.1895311 & 34.3424998 & 36.1499998 & -14.459999 & $(1 \overline{1} \overline{1}) /[\overline{1} 1 \overline{2}]$ \\
\hline \multirow[t]{2}{*}{4.075} & 0.1901234 & 36.1499998 & 34.3424998 & -14.459999 & $(1 \overline{1} 1) /[\overline{1} 12]$ \\
\hline & 0.1901234 & 34.3424998 & 36.1499998 & -14.459999 & $(1 \overline{1} \overline{1}) /[\overline{1} 1 \overline{2}]$ \\
\hline \multirow[t]{2}{*}{4.08} & 0.1913624 & 36.1499998 & 34.3424998 & -14.459999 & $(1 \overline{1} 1) /[\overline{1} 12]$ \\
\hline & 0.1913624 & 34.3424998 & 36.1499998 & -14.459999 & $(1 \overline{1} \overline{1}) /[\overline{1} 1 \overline{2}]$ \\
\hline \multirow[t]{2}{*}{4.085} & 0.1921886 & 36.1499998 & 34.3424998 & -14.459999 & $(111) /[112]$ \\
\hline & 0.1921886 & 34.3424998 & 36.1499998 & -14.459999 & $(1 \overline{1} 1) /[\overline{1} 1 \overline{2}]$ \\
\hline \multirow[t]{2}{*}{4.09} & 0.1918851 & 36.1499998 & 34.3424998 & -14.459999 & $(111) /[112]$ \\
\hline & 0.1918851 & 34.3424998 & 36.1499998 & -14.459999 & $(1 \overline{1} 1) /[\overline{1} 1 \overline{2}]$ \\
\hline \multirow[t]{2}{*}{4.095} & 0.1930605 & 36.1499998 & 34.3424998 & -14.459999 & $(1 \overline{1} 1) /[\overline{1} 12]$ \\
\hline & 0.1930605 & 34.3424998 & 36.1499998 & -14.459999 & $(1 \overline{1} \overline{1}) /[\overline{1} 1 \overline{2}]$ \\
\hline \multirow[t]{2}{*}{4.10} & 0.1947941 & 36.1499998 & 34.3424998 & -14.459999 & $(1 \overline{1} 1) /[\overline{1} 12]$ \\
\hline & 0.1947941 & 34.3424998 & 36.1499998 & -14.459999 & $(1 \overline{1} \overline{1}) /[\overline{1} 1 \overline{2}]$ \\
\hline \multirow[t]{2}{*}{4.105} & 0.1966424 & 36.1499998 & 34.3424998 & -14.459999 & $(1 \overline{1} 1) /[\overline{1} 12]$ \\
\hline & 0.1966424 & 34.3424998 & 36.1499998 & -14.459999 & $(1 \overline{1} \overline{1}) /[\overline{1} 1 \overline{2}]$ \\
\hline
\end{tabular}

Table 4.1: The predicted nucleation sites and slip system of dislocation with the maximum $\mathrm{N}_{m, l}$ value for $\mathrm{Cu}(001)$. These results agree with the actual nucleation sites $(36.15,34.34,-14.46)$ and $(34.34,36.15,-14.46)$ shown in Figure 4.10, and slip systems $(1 \overline{1} \overline{1}) / \frac{1}{6}[\overline{1} 1 \overline{2}]$ and $(1 \overline{1} 1) / \frac{1}{6}[\overline{1} 12]$. 
Table 4.1 shows the numerical prediction result using the "Acharya/Miller" criterion for $\mathrm{Cu}(001)$ model. It includes the columns of indenter displacement, the maximum $\mathrm{N}_{m, l}$ value, nucleation site coordinates, and the predicted slip system (slip plane and Burgers vector). All these values are obtained before the nucleation event from the moment of $4.0 \AA$ depth to $4.105 \AA$ depth, the maximum $\mathrm{N}_{m, l}$ value among all the atoms happens at the same sites and the characters of slip system keep as Shockley partial dislocations (i.e., $(1 \overline{1} 1) / \frac{1}{6}[\overline{1} 12]$ and $(1 \overline{1} \overline{1}) / \frac{1}{6}[\overline{1} 1 \overline{2}]$ ) for the period of the simulation time. Comparing with the actual dislocations after nucleation, the predicted site of nucleation and dislocation type are found to agree exactly with the actual dislocations (indicated by slip vector, and nucleate as same Shockley partial dislocations $(1 \overline{1} 1) / \frac{1}{6}[\overline{1} 12]$ and $\left.(1 \overline{1} \overline{1}) / \frac{1}{6}[\overline{1} 1 \overline{2}]\right)$. Therefore, the content of table 4.1 demonstrates that the "Acharya/Miller" criterion can be proved to be an efficient predictor with high quality.

\begin{tabular}{|l|l|l|l|}
\hline \multicolumn{2}{|l|}{$\begin{array}{l}\text { Simulation } \\
\text { model }\end{array}$} & $\begin{array}{l}\text { Indenter load at nucleation } \\
(\mathrm{eV} / \AA)\end{array}$ & $\mathrm{N}_{\text {crit }}\left(\mathrm{eV} / \AA^{4}\right)$ \\
\hline \multirow{3}{*}{$\mathrm{Ni}$} & $(001)$ & $100.5 \pm 2.0$ & \\
\cline { 2 - 4 } & $(110)$ & $240.0 \pm 2.0$ & $0.21 \pm 0.02$ \\
\cline { 2 - 4 } & $(111)$ & $330.0 \pm 2.0$ & $0.22 \pm 0.02$ \\
\hline \multirow{3}{*}{$\mathrm{Cu}$} & $(001)$ & $63.5 \pm 2.0$ & $0.22 \pm 0.02$ \\
\cline { 2 - 4 } & $(110)$ & $112.0 \pm 2.0$ & $0.16 \pm 0.02$ \\
\cline { 2 - 4 } & $(111)$ & $184.5 \pm 2.0$ & $0.15 \pm 0.02$ \\
\hline
\end{tabular}

Table 4.2: Table of simulation results

Table 4.2 shows the values of $\mathrm{N}_{\text {crit }}$ and indenter loads at nucleation for two fcc crystalline materials ( $\mathrm{Ni}$ and $\mathrm{Cu}$ ) with three different orientations ( (001), (110) and (111) ). It is obvious that the $\mathrm{N}_{\text {crit }}$ is a material related parameter (roughly keeps as constant within each set of orientations for each material), and the indenter load 
varies with simulation models because of the influence of mechanical properties (e.g., hardness).

The detailed simulation results for other models are described in Appendix A. Comparing all these results, the nucleated Shockley partial dislocations are found always occur on $\{111\}$ planes, whereas the dislocation structure and corresponding displacement at nucleation are different for each orientation. The fact is proved that the abrupt jump of $\mathrm{N}_{m, l}$ value can be used to accurately predict the location and type of defects in all models. Therefore, current simulations successfully examine the prediction capability of the "Acharya/Miller" criterion.

In order to reveal the agreement of the predicted locations and the sites of actual dislocation at nucleation, an example in Figure 4.11 is provided to clearly show the actual and predicted nucleation sites at the same moment for the $\mathrm{Cu}(110)$ model. The top snapshot is the slip vector distribution and the bottom snapshot shows the $\mathrm{N}_{m, l}$ value. Comparing these two snapshots, it is clear that the maximum $\mathrm{N}_{m, l}$ value exactly occurs at the same sites as the maximum magnitude of the slip vector. This observation also demonstrates that the slip character determined by the $\mathrm{N}_{m, l}$ value is in excellent agreement with the actual slip character. 


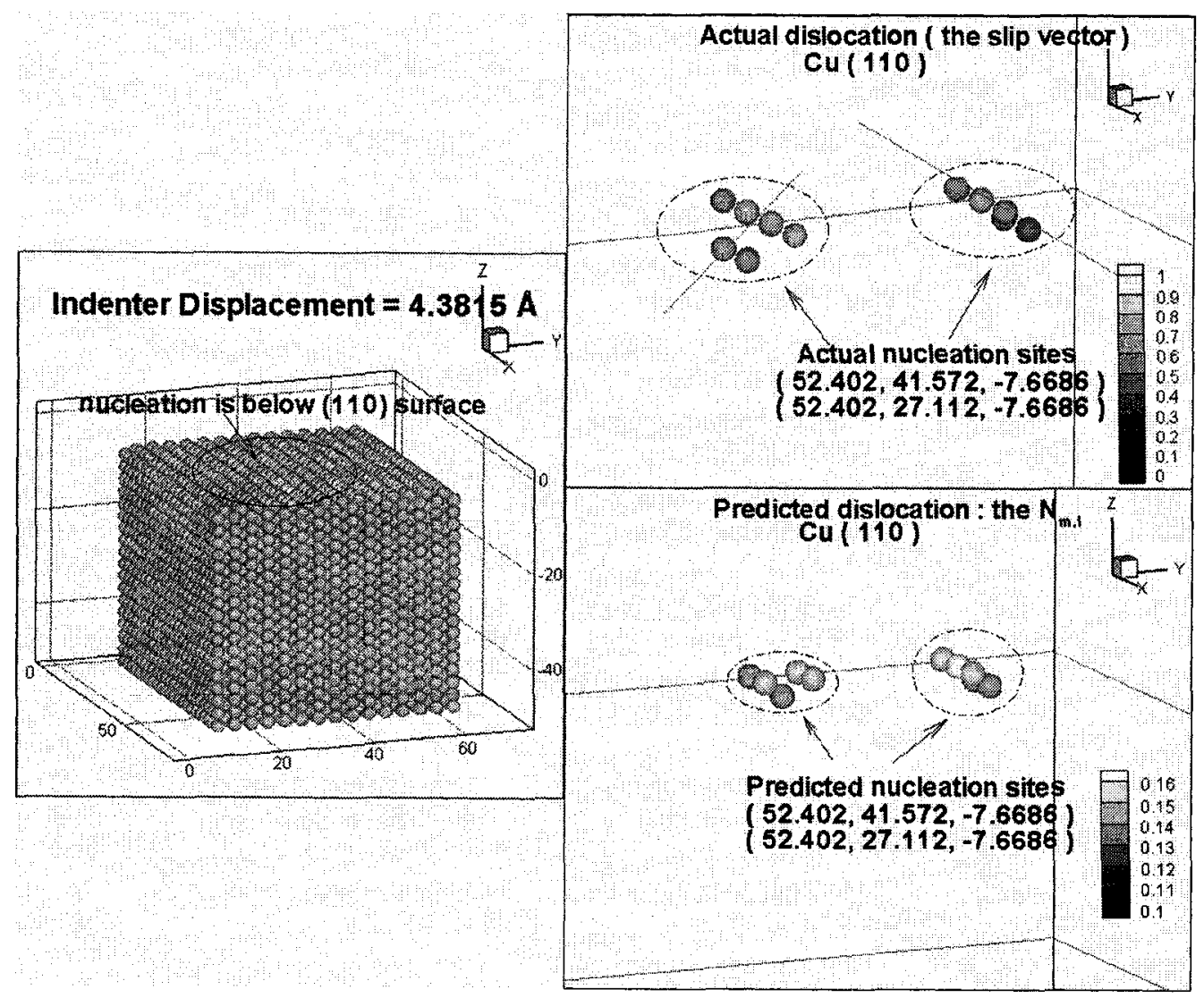

Figure 4.11: The comparison between the $\mathrm{N}_{m, l}$ and slip vector distribution below $\mathrm{Cu}$ (110) indentation plane during dislocation nucleation in crystal at the same simulation moment. 


\section{Chapter 5}

\section{Conclusion}

The purpose of this thesis was to test the capability of a stress gradient based criterion (i.e., the so called "Acharya/Miller" criterion), and try to understand the mechanical conditions which lead to dislocation nucleation for crystalline materials. The nucleation of individual dislocations and their interaction is crucial to understand the initial stage of plasticity deformation. This thesis presents a brief summary of dislocation structures for fcc crystal structure and a method used to predict the event of dislocation nucleation with nano-indentation. The main emphasis of the research is the examination of the "Acharya/Miller" criterion by comparing the actual dislocation results and the predictions.

This work presents the $3 \mathrm{D}$ atomic simulations of nano-indentation by a spherical indenter to quantify the critical state of dislocation nucleation for single fcc crystal structures. Predictions are given to demonstrate when and where the dislocation will nucleate within the crystal structure, and what slip mode the nucleated dislocations will take. The pictorial results of atomic simulation show that the individual dislocation originally generates below the indentation surface and inside the contact area, that Shockley partial dislocations bounding each stacking fault are the dislocations formed, and that they prefer to glide on the (111) type planes.

To gain insight into the influence of anisotropy, three types of simulation model are 
created with different crystal orientation (rotating the fcc crystal structure and separately making the indentation surface as (001), (110) and (111) planes). Reviewing the results of nano-indentation simulation, the effect of anisotropy is found to be a complicating factor to influence the result. Nearly all metals are to some degree elastically anisotropic, especially for the pure $\mathrm{Cu}$ and $\mathrm{Ni}$, the most anisotropic fcc metals. The simulation results suggest that there is a significant effect of crystal orientation existing on the configuration of slip systems and indicate that anisotropic effects play a significant role in nano-indentation.

In this thesis, 3D MD simulations are performed to verify the "Acharya/Miller" criterion quantitatively. The crystal orientation has an effect on the slip mode (slip plane and Burgers vector), the configuration of dislocation structure, the indenter depth corresponding to a nucleation event, etc. This thesis proves that there exists a material property $\mathrm{N}_{\text {crit }}$, which is independent of the orientation of the material and can be used to predict the events of dislocation nucleation correctly. the $\mathrm{N}_{\text {crit }}$ of $\mathrm{Cu}$ is suggested as $0.16 \pm 0.02\left(\mathrm{eV} / \AA^{4}\right)$ and $\mathrm{N}_{\text {crit }}$ of $\mathrm{Ni}$ is $0.22 \pm 0.02\left(\mathrm{eV} / \AA^{4}\right)$. Nucleation occurs when a stress-gradient related value $\mathrm{N}_{m, l}$ reaches to $\mathrm{N}_{\text {crit }}$. The maximum $\mathrm{N}_{m, l}$ prior to a nucleation event can predict the character of the dislocations and the location of the nucleation. Dislocation nucleation and gliding happens as Shockley partial dislocations, $\{111\} /\langle 112\rangle$.

In summary, these direct MD simulations, conducted for a small system at a comparatively high indentation loading rate (e.g., about $5 \mathrm{~m} / \mathrm{s}$ ), verify the prediction capability of the stress-gradient based criterion in fec crystals $(\mathrm{Cu}$ and $\mathrm{Ni})$.

\section{Recommendations for Future Work}

Although the scope of this thesis is limited to the analysis of fcc crystalline materials, it can be extended to analyzing other crystalline structures (e.g., bcc, hcp) and heterogeneous nucleation systems. It is excepted that the "Acharya/Miller" criterion 
will help to predict the onset of plastic deformation and avoid further material damage in these systems as well.

In this thesis, the current dislocation nucleation study is restricted to the idealized behavior at the temperature of $0 K$ to avoid the specific effects of thermally activated processes. In the future, the study of dislocation nucleation could be focused on the effects of temperature to the actual simulations and to the value of $\mathrm{N}_{\text {crit }}$.

Though the "Acharya/Miller" criterion had already been examined in 2D discrete dislocation models using CADD method, an extension should be considered to use this criterion in $3 \mathrm{D}$ discrete dislocation models to represent the nucleation of defects in such models. 


\section{Appendix A: More simulation results}

\section{Ni (001) model}

Figure 5.1 shows the indenter load versus displacement curve and the $\mathrm{N}_{m, l}$ versus displacement curve for $\mathrm{Ni}(001)$ model, used to demonstrate the prediction capability of the "Acharya/Miller" criterion for nucleation events. As with $\mathrm{Cu}(001)$, the $\mathrm{N}_{m, l}$ value reaches to the $\mathrm{N}_{\text {crit }}$ value and jumps to a large value at the same moment as the drop of the indenter load. Comparing the results of these two models, the indenter displacements corresponding to the nucleation moment are different (i.e., $4.0 \AA$ for $\mathrm{Cu}$, and $4.2 \AA$ for $\mathrm{Ni}$ ) because of the different mechanical properties. Specifically, $\mathrm{N}_{\text {crit }}$ of $\mathrm{Ni}$ is $0.21 \pm 0.02\left(\mathrm{eV} / \AA^{4}\right)$.

As with $\mathrm{Cu}(001)$, the top part of Figure 5.2 is the visualization of $\mathrm{N}_{m, l}$ distribution inside the $\mathrm{Ni}(001)$ crystal. The maximum values of $\mathrm{N}_{m, l}$ always happens as Shockley partial dislocations $(1 \overline{1} \overline{1}) / \frac{1}{6}[\overline{1} 1 \overline{2}]$ and $\left.(1 \overline{1} 1) / \frac{1}{6}[\overline{1} 12]\right)$ just prior to the dislocation nucleation event. The bottom part of Figure 5.2 represents the dislocations structure indicated by the slip vector after the nucleation event at indenter depth $4.3 \AA$. It is seen that the $\mathrm{V}$-shaped half-loop structure is found again. Comparing these two parts, it is obvious that the predicted nucleation site and slip systems exactly agree with the actual results. The facts show that the "Acharya/Miller" criterion has the prediction capability for different fcc crystalline materials.

Like Table 4.1, Table 5.1 also shows the numerical prediction result for $\mathrm{Ni}(001)$ model. All these values are collected before the nucleation event from the moment of $4.1 \AA$ to $4.2 \AA$ indenter depth, the maximum $\mathrm{N}_{m, l}$ value among all the atoms happens at the same location and the characters of the slip systems are Shockley partials (i.e., (111) $/ \frac{1}{6}[\overline{1} 12]$ and $\left.(1 \overline{1} \overline{1}) / \frac{1}{6}[\overline{1} 1 \overline{2}]\right)$ for this period of the simulation time, these results are same as the actual dislocations. The content of Table 5.1 again proves the 
"Acharya/Miller" criterion as an efficient predictor. Comparing the results of $\mathrm{Cu}$ (001) and $\mathrm{Ni}(001)$ models, although the nucleation locations are different because of the material hardness, another conclusion can be obtained that the configuration of dislocation structure and the slip systems are same because of the same crystalline orientation.

\begin{tabular}{|l|l|l|l|l|l|}
\hline \multirow{2}{*}{ Depth } & \multirow{2}{*}{ Max N $_{m, l}$} & \multicolumn{4}{|l|}{ Predicted nucleation site and dislocation character } \\
\cline { 3 - 6 } & & Coor_x & Coor_y & Coor_z & Slip system \\
\hline 4.15 & 0.2384133 & 35.20000 & 33.44000 & -14.08000 & $(1 \overline{1} 1) /[\overline{1} 12]$ \\
& 0.2384133 & 33.44000 & 35.20000 & -14.08000 & $(1 \overline{1} \overline{1}) /[\overline{1} 1 \overline{2}]$ \\
\hline 4.155 & 0.2411232 & 35.20000 & 33.44000 & -14.08000 & $(1 \overline{1} 1) /[\overline{1} 12]$ \\
& 0.2411232 & 33.44000 & 35.20000 & -14.08000 & $(1 \overline{1} \overline{1}) /[\overline{1} 1 \overline{2}]$ \\
\hline 4.16 & 0.2432186 & 35.20000 & 33.44000 & -14.08000 & $(1 \overline{1} 1) /[\overline{1} 12]$ \\
& 0.2432186 & 33.44000 & 35.20000 & -14.08000 & $(1 \overline{1} \overline{1}) /[\overline{1} 1 \overline{2}]$ \\
\hline 4.165 & 0.2470928 & 35.20000 & 33.44000 & -14.08000 & $(1 \overline{1} 1) /[\overline{1} 12]$ \\
& 0.2470928 & 33.44000 & 35.20000 & -14.08000 & $(1 \overline{1} \overline{1}) /[\overline{1} 1 \overline{2}]$ \\
\hline 4.17 & 0.2494909 & 35.20000 & 33.44000 & -14.08000 & $(1 \overline{1} 1) /[\overline{1} 12]$ \\
& 0.2494909 & 33.44000 & 35.20000 & -14.08000 & $(1 \overline{1} \overline{1}) /[\overline{1} 1 \overline{2}]$ \\
\hline 4.175 & 0.2536904 & 35.20000 & 33.44000 & -14.08000 & $(1 \overline{1} 1) /[\overline{1} 12]$ \\
& 0.2536904 & 33.44000 & 35.20000 & -14.08000 & $(1 \overline{1} \overline{1}) /[\overline{1} 1 \overline{2}]$ \\
\hline 4.18 & 0.2586947 & 35.20000 & 33.44000 & -14.08000 & $(1 \overline{1} 1) /[\overline{1} 12]$ \\
& 0.2586947 & 33.44000 & 35.20000 & -14.08000 & $(1 \overline{1} \overline{1}) /[\overline{1} 1 \overline{2}]$ \\
\hline 4.185 & 0.2637058 & 35.20000 & 33.44000 & -14.08000 & $(1 \overline{1} 1) /[\overline{1} 12]$ \\
& 0.2637058 & 33.44000 & 35.20000 & -14.08000 & $(1 \overline{1} \overline{1}) /[\overline{1} 1 \overline{2}]$ \\
\hline 4.19 & 0.2693144 & 35.20000 & 33.44000 & -14.08000 & $(1 \overline{1} 1) /[\overline{1} 12]$ \\
& 0.2693144 & 33.44000 & 35.20000 & -14.08000 & $(1 \overline{1} \overline{1}) /[\overline{1} 1 \overline{2}]$ \\
\hline 4.195 & 0.2782198 & 35.20000 & 33.44000 & -14.08000 & $(1 \overline{1} 1) /[\overline{1} 12]$ \\
& 0.2782198 & 33.44000 & 35.20000 & -14.08000 & $(1 \overline{1} \overline{1}) /[\overline{1} 1 \overline{2}]$ \\
\hline 4.2 & 0.2859840 & 36.96000 & 33.44000 & -15.84000 & $(1 \overline{1} \overline{1}) /[\overline{1} 12]$ \\
& 0.2859840 & 33.44000 & 36.96000 & -15.84000 & $(1 \overline{1} \overline{1}) /[\overline{1} 1 \overline{2}]$ \\
\hline
\end{tabular}

Table 5.1: The predicted nucleation sites and slip system of dislocation with the maximum $\mathrm{N}_{m, l}$ value for $\mathrm{Ni}(001)$. These results agree with the actual nucleation sites $(35.2,33.44,-14.08)$ and $(33.44,35.2,-14.08)$ atom sites shown in Figure 5.2, and slip system $(1 \overline{1} \overline{1}) / \frac{1}{6}[\overline{1} 1 \overline{2}]$ and $(1 \overline{1} 1) / \frac{1}{6}[\overline{1} 12]$. 

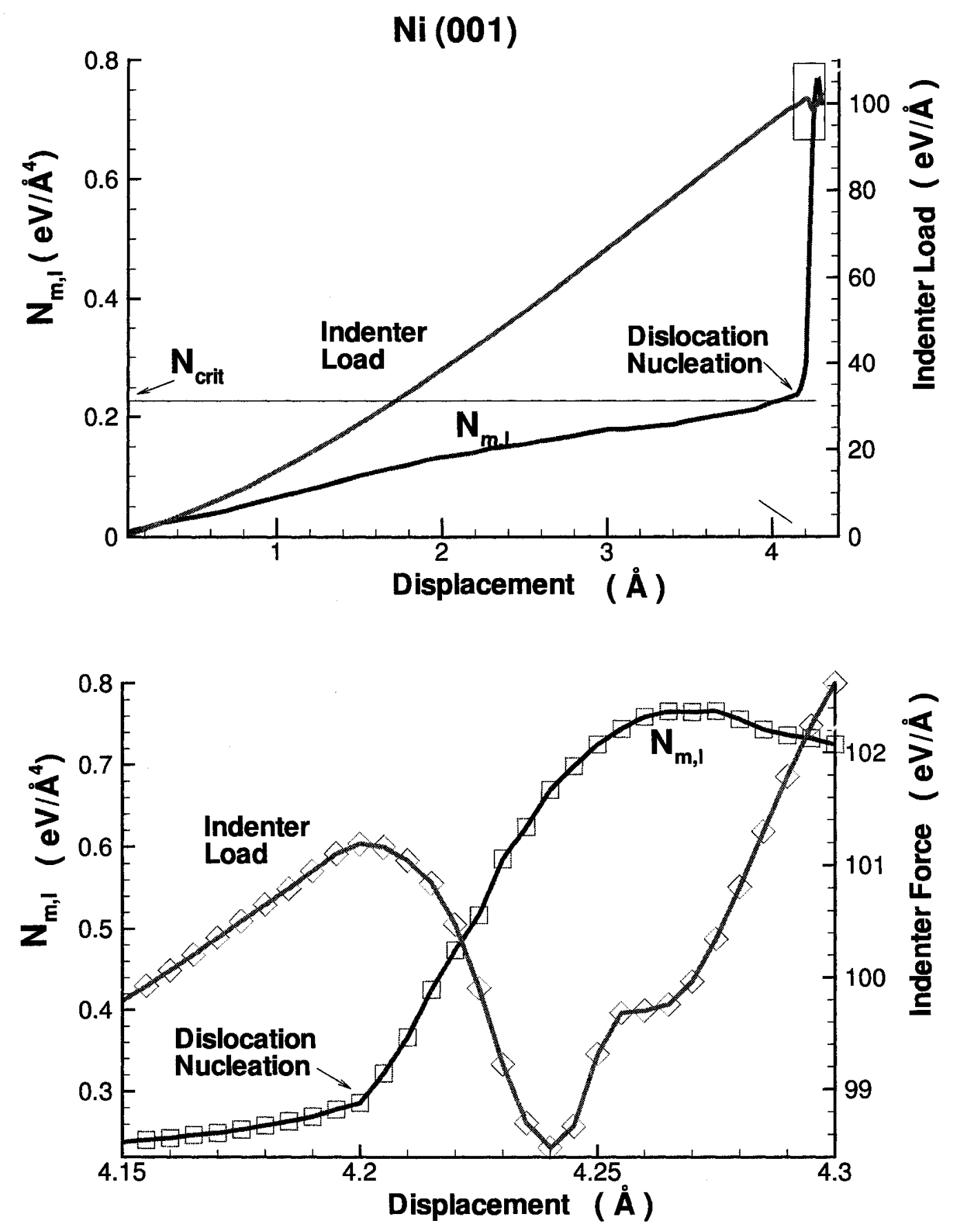

Figure 5.1: The correlation of two curves: the indenter load versus displacement curve and the maximum $\mathrm{N}_{m, l}$ versus displacement curves for $\mathrm{Ni}(001)$ model. The lower figure is an enlargement of the late stages of the top graphs. 

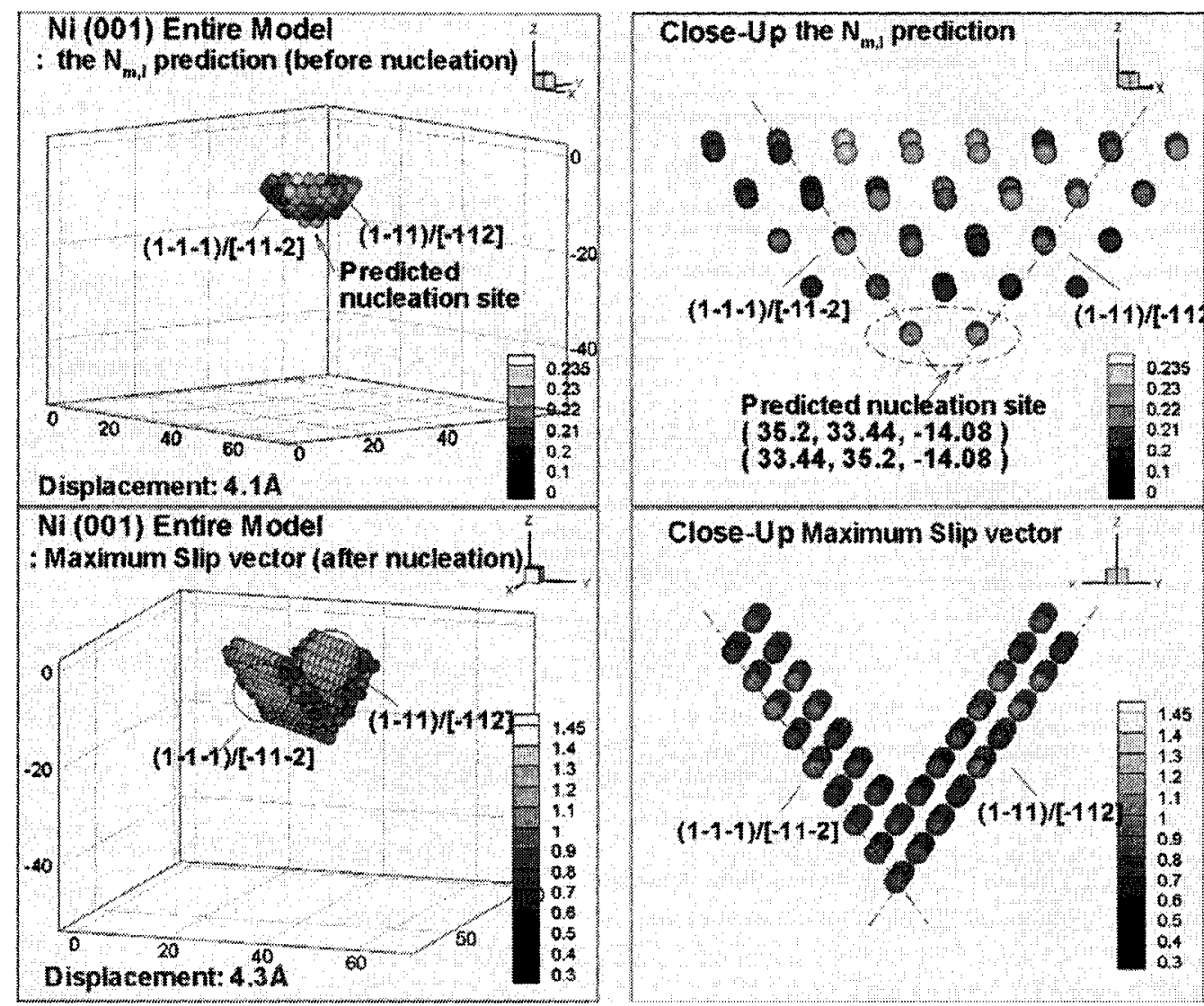

Figure 5.2: The comparison of prediction result and actual dislocation is shown for the $\mathrm{Ni}(001)$ model. The bottom part of the figure is the slip vector distribution immediately after the nucleation event, and top part of the figure is the maximum $\mathrm{N}_{m, l}$ value distribution throughout the atom slab prior to the nucleation. 


\section{Cu (110) model}

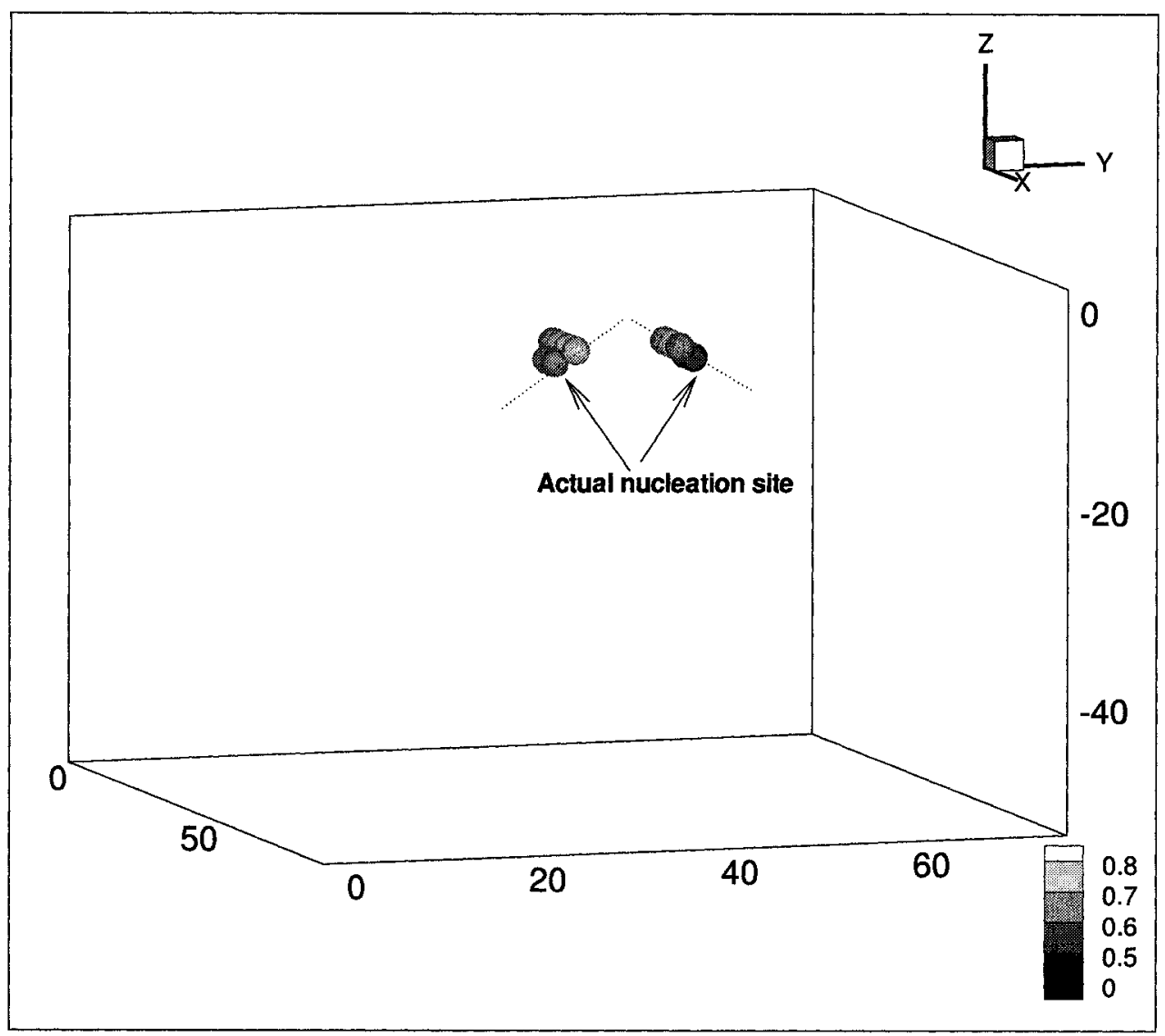

Figure 5.3: The snapshot of dislocation nucleation is presented by the slip vector for $\mathrm{Cu}(110)$ model.

Figure 5.3 shows a snapshot of the nucleation event presented by the slip vector near the first plastic yield point for $\mathrm{Cu}(110)$ model. The two dislocation embryos nucleate on two inclined $\{111\}$ type of slip planes below the indentation surface and inside the contact area, expand into two partial dislocation loops, and then extend into the solid. 
Figure 5.4 shows the contour of the slip vector with a side view (a rotation between the [111] and [11̄2] directions) to indicate the character of the Shockley partial dislocations at the moment of $4.41 \AA$ indenter depth after the dislocation nucleation event. The dislocation loops grow in size and intersect with the loops on the adjacent planes to form an inverted V-shape dislocation structure. It is clear that a Shockley partial dislocation bounding a stacking fault is expected to glide easily on the $\{111\}$ close-packed planes. The left plane is found to be the (111) slip plane and the right is the (ī1) slip plane, and the corresponding slip vectors are $\frac{1}{6}[\overline{1} \overline{1} 2]$ and $\frac{1}{6}[\overline{2} 1 \overline{1}]$.

Figure 5.5 shows the stacking faults of Shockley partial dislocations in the same side view as the Figure 5.4. The maximum magnitude of slip vector is around $1.47 \AA$ for $\mathrm{Cu}$ and $1.66 \AA$ for $\mathrm{Ni}$. Its components are also consistent with the $\frac{1}{6}\langle 112\rangle$ partial dislocations on the $\{111\}$ slip planes in fcc metals.

Figure 5.6 shows the front view (along the [111] direction), it is obvious that two parallel layers of atoms with large magnitude of the slip vector on each side of the slip plane are shown, and the slip vector on the two layers have the same magnitude of slip vector along opposite slip directions. It is also obvious that the atoms inside the stacking faults have larger magnitude of slip vector than the others. 


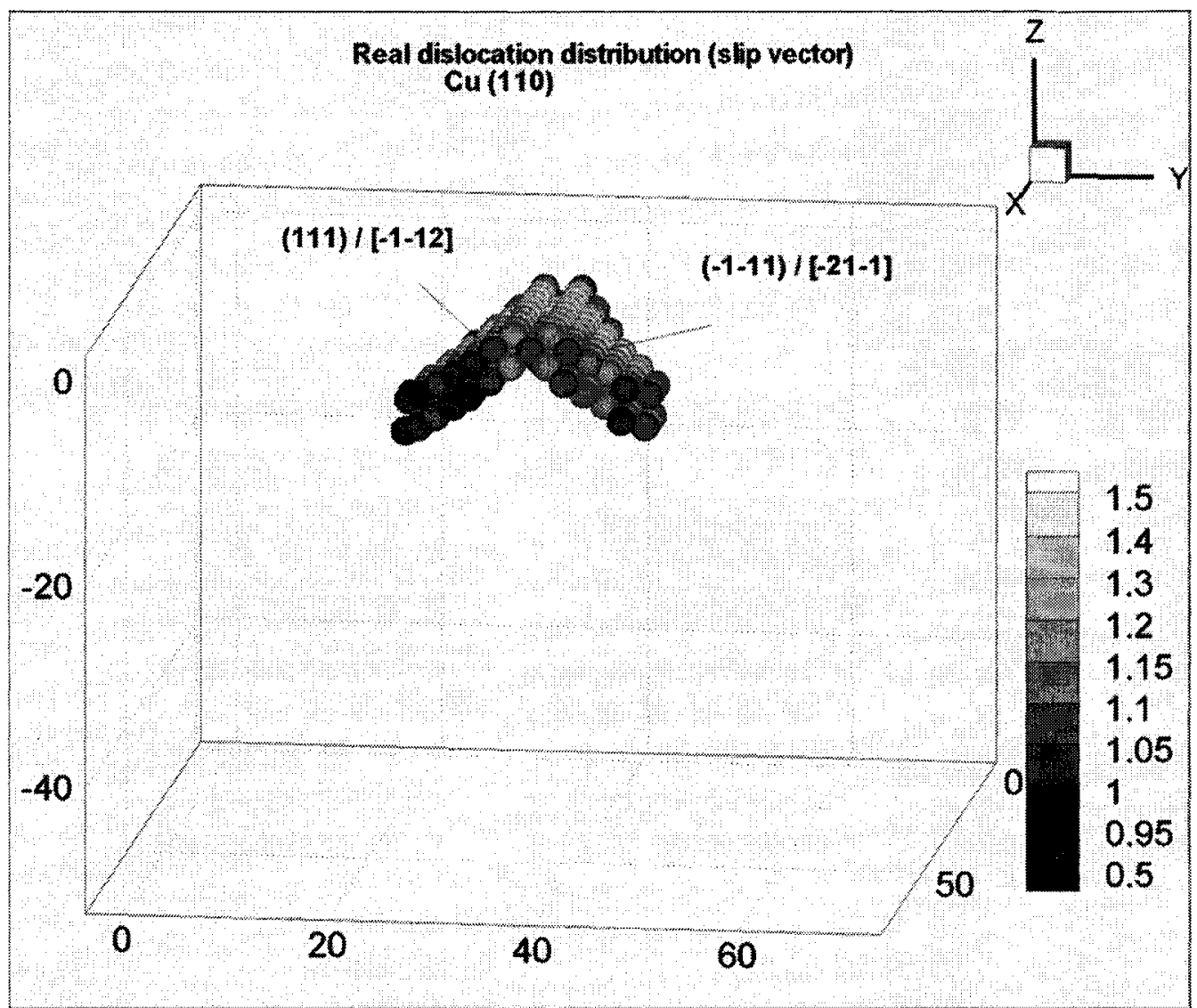

Figure 5.4: Contour of slip vector on slip plane to demonstrate the actual dislocation structure beneath the indentation (110) surface. 


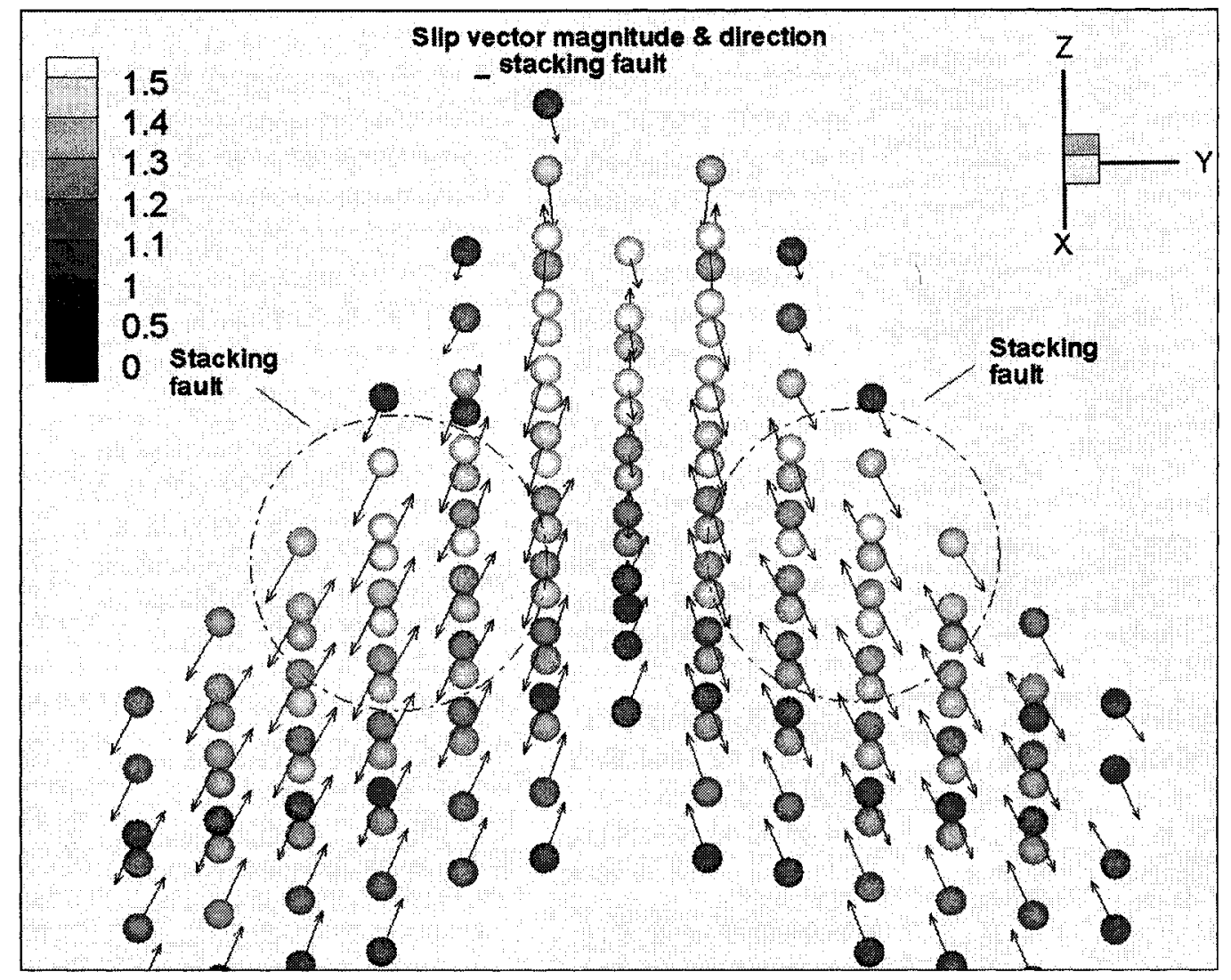

Figure 5.5: Vector graph of slip vector on slip plane to demonstrate the magnitude and direction of Burgers vector for $\mathrm{Cu}(110)$ model. 


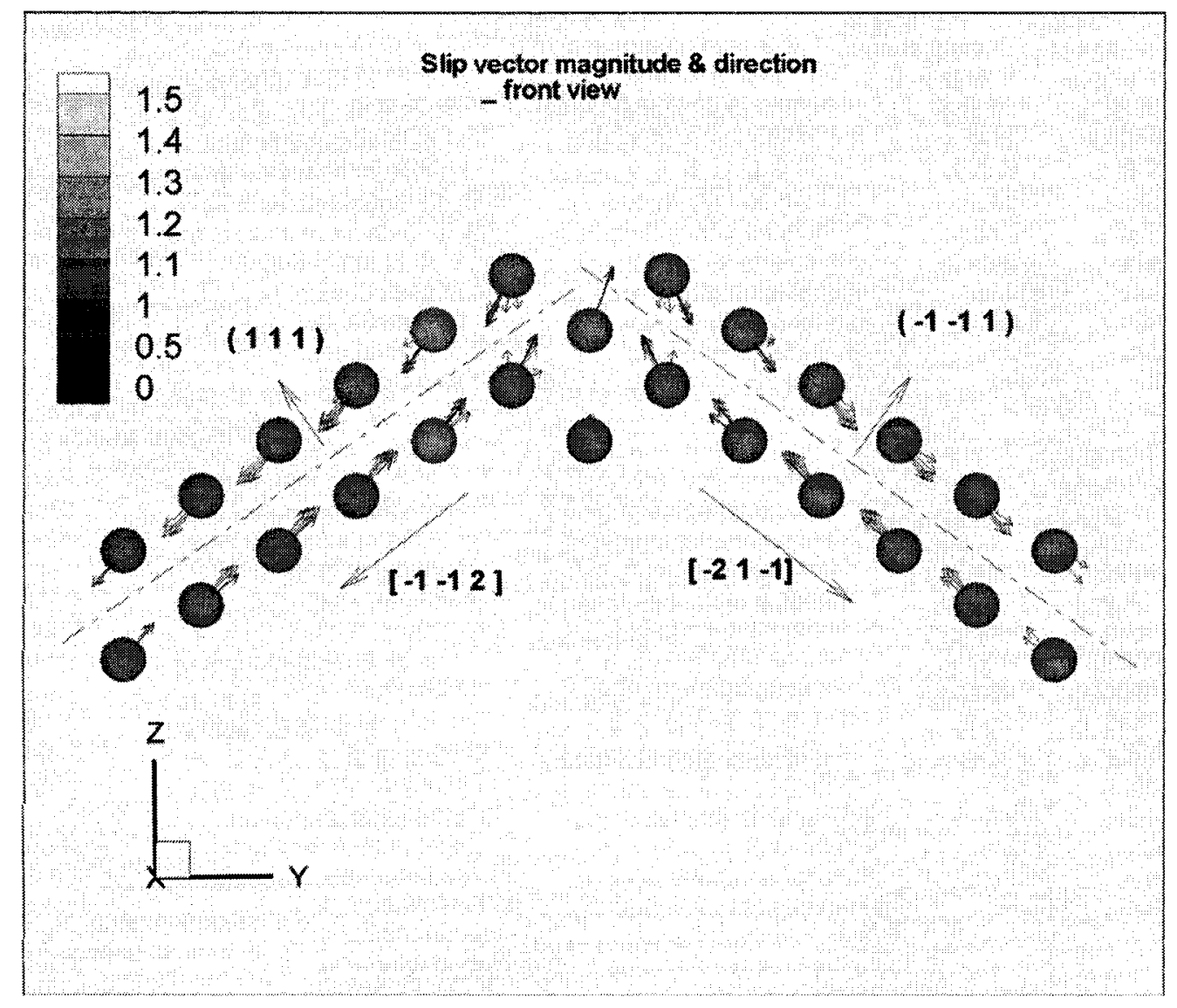

Figure 5.6: Front view of actual dislocation structure to demonstrate the magnitude and direction of Burgers vector for $\mathrm{Cu}$ (110) model. 

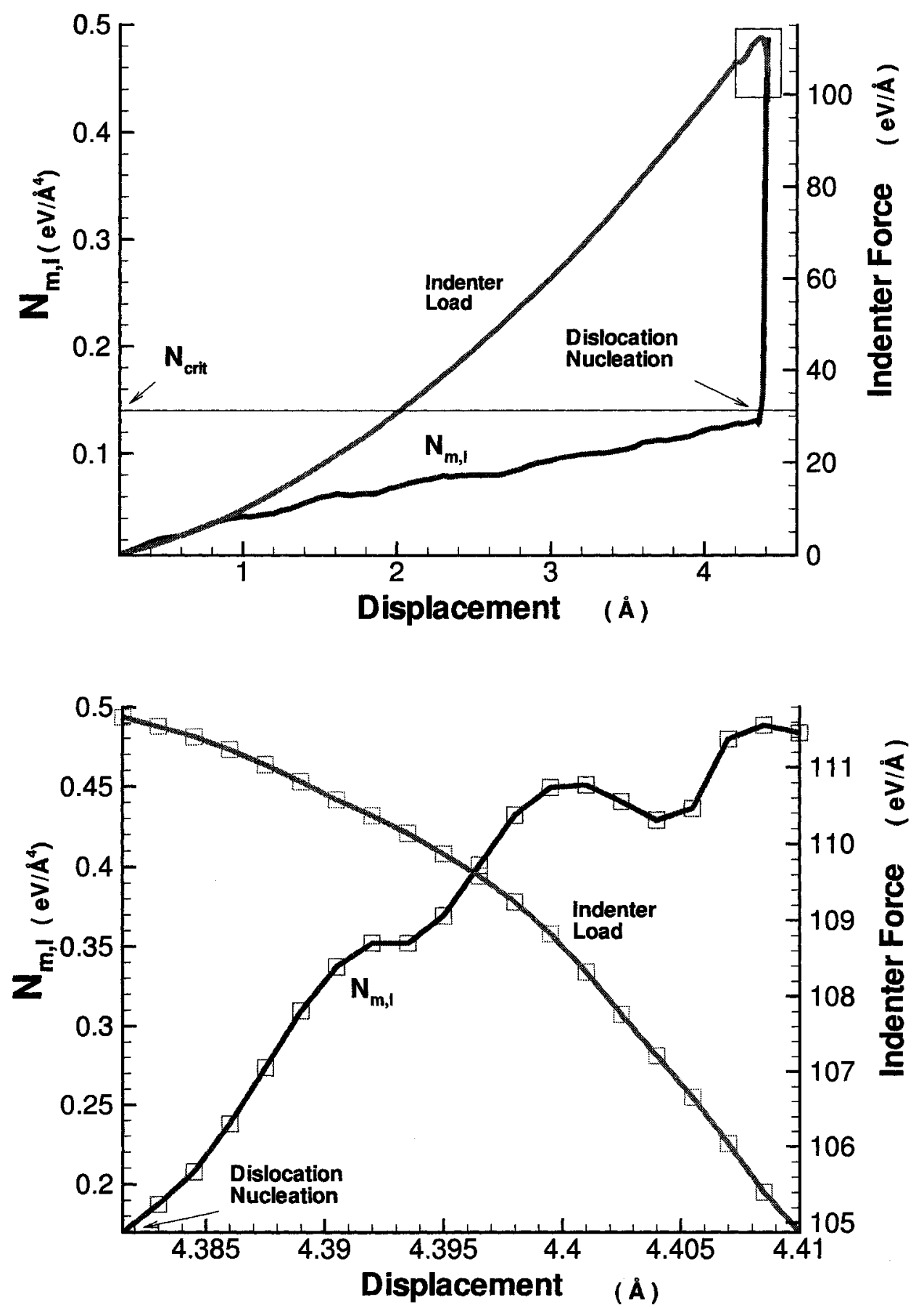

Figure 5.7: The correlation of the indenter load versus displacement curve and the maximum $\mathrm{N}_{m, l}$ versus displacement curve for the $\mathrm{Cu}(110)$ model. The lower figure is an enlargement of the late stages of the top graphs. 


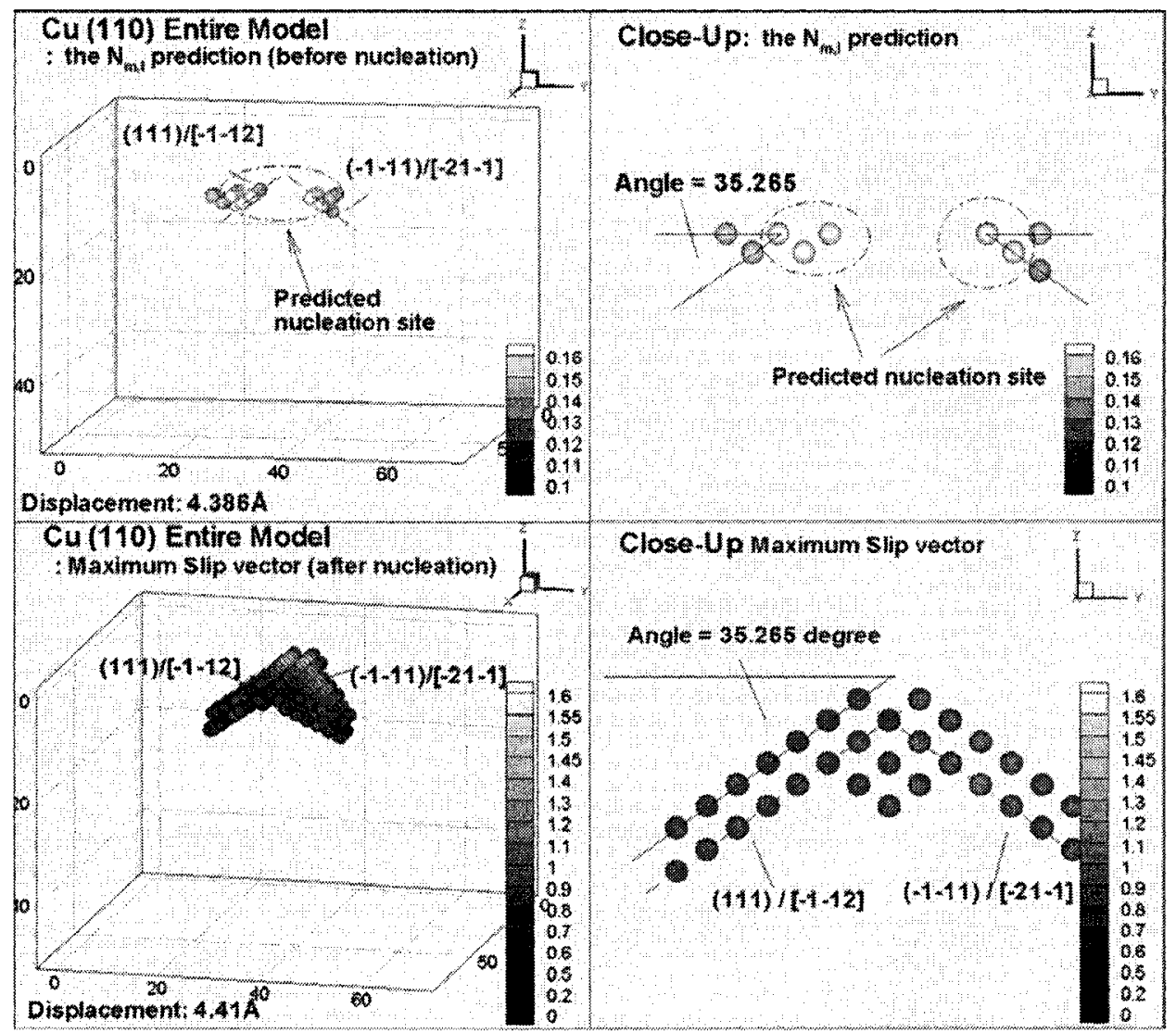

Figure 5.8: The comparison of prediction result and actual dislocation is shown for $\mathrm{Cu}(110)$ model. The top part of the figure is a figure of slip vector distribution immediately after the nucleation event, and bottom part of the figure is the maximum $\mathrm{N}_{m, l}$ value distribution throughout the atom slab. 
Figure 5.7 shows the indenter load versus displacement curve and the $\mathrm{N}_{m, l}$ versus displacement curve for the $\mathrm{Cu}(110)$ model. The indenter load drops is found at an indentation depth of $4.38 \AA$, when the onset of dislocation nucleation is first detected via the "Acharya/Miller" criterion. The corresponding $\mathrm{N}_{m, l}$ value (it can be regarded as the $\mathrm{N}_{\text {crit }}$ value) is about $0.15 \pm 0.02\left(\mathrm{eV} / \AA^{4}\right)$ for $\mathrm{Cu}(110)$. The $\mathrm{N}_{\text {crit }}$ value obtained from $\mathrm{Cu}(001)$ model is around $0.16 \pm 0.02\left(\mathrm{eV} / \mathrm{o}^{4}\right)$, which is very close to the $\mathrm{N}_{\text {crit }}$ value obtained from this $\mathrm{Cu}(110)$ simulation, and the difference between them is less than $5 \%$. These facts prove that the $\mathrm{N}_{\text {crit }}$ is material related property and independent with crystal orientations.

Figure 5.8 shows the visualization of the actual dislocation nucleation indicated by the slip vector and the $\mathrm{N}_{m, l}$ predictions for the $\mathrm{Cu}$ (110) model. The maximum value of $\mathrm{N}_{m, l}$ occurs for Shockley partial dislocations $(111) / \frac{1}{6}[\overline{1} \overline{1} 2]$ and $(\overline{1} \overline{1} 1) / \frac{1}{6}[\overline{2} 1 \overline{1}]$ when the indenter displacement is around $4.36 \AA$ just prior to the nucleation event. The actual dislocations are found to be the same Shockley partials predicted using the maximum $\mathrm{N}_{m, l}$ value. It indicates that the dislocation character and nucleation site can be correctly predicted with this criterion for $\mathrm{Cu}(110)$ model.

Table 5.2 shows the numerical prediction results for the $\mathrm{Cu}(110)$ model using the "Acharya/Miller" criterion. All these values are recorded before the nucleation event from the moment of $4.38 \AA$ depth, the maximum $N_{m, l}$ value happens at the same sites as the actual nucleation shown in Figure 5.3 and the characters of the slip system are $(111) / \frac{1}{6}[\overline{1} \overline{1} 2]$ and $(\overline{1} \overline{1} 1) / \frac{1}{6}[\overline{2} 1 \overline{1}]$ Shockley partial dislocations, which agree with the actual dislocations presented by the slip vector. The content of table 5.2 demonstrates that the "Acharya/Miller" criterion can be successfully used for the $\mathrm{Cu}(110)$ model. 


\begin{tabular}{|c|c|c|c|c|c|}
\hline \multirow{2}{*}{ Depth } & \multirow{2}{*}{$\operatorname{Max} \mathrm{N}_{m, l}$} & \multicolumn{4}{|c|}{ Predicted nucleation site and dislocation character } \\
\hline & & Coor_x & Coor_y & Coor_z & Slip system \\
\hline \multirow[t]{2}{*}{4.3815} & 0.1698858 & 52.401915 & 41.572499 & -7.6685745 & $(111) /[\overline{1} \overline{1} 2]$ \\
\hline & 0.1563014 & 52.401915 & 27.112499 & -7.6685745 & $(\overline{1} 11) /[\overline{2} 1 \overline{1}]$ \\
\hline \multirow[t]{2}{*}{4.383} & 0.1874804 & 52.401915 & 41.572499 & -7.6685745 & $(111) /[112]$ \\
\hline & 0.1799228 & 52.401915 & 27.112499 & -7.6685745 & $(\overline{1} 11) /[\overline{2} 1 \overline{1}]$ \\
\hline \multirow[t]{2}{*}{4.3845} & 0.2078065 & 52.401915 & 41.572499 & -7.6685745 & $(111) /[112]$ \\
\hline & 0.2049432 & 52.401915 & 27.112499 & -7.6685745 & $(\overline{1} 11) /[\overline{2} 1 \overline{1}]$ \\
\hline \multirow[t]{2}{*}{4.386} & 0.2194007 & 52.401915 & 41.572499 & -7.6685745 & $(111) /[\overline{1} 12]$ \\
\hline & 0.2378989 & 52.401915 & 27.112499 & -7.6685745 & $(\overline{1} \overline{1} 1) /[\overline{2} 1 \overline{1}]$ \\
\hline \multirow[t]{2}{*}{4.3875} & 0.2312463 & 52.401915 & 41.572499 & -7.6685745 & $(111) /[\overline{1} \overline{1} 2]$ \\
\hline & 0.2737288 & 52.401915 & 27.112499 & -7.6685745 & $(\overline{1} 11) /[\overline{2} 1 \overline{1}]$ \\
\hline \multirow[t]{2}{*}{4.389} & 0.2413515 & 53.680010 & 43.379999 & -8.9466700 & $(111) /[\overline{1} \overline{1} 2]$ \\
\hline & 0.3097773 & 52.401915 & 27.112499 & -7.6685745 & $(\overline{1} \overline{1} 1) /[\overline{2} 1 \overline{1}]$ \\
\hline \multirow[t]{2}{*}{4.3905} & 0.2593504 & 53.680010 & 43.379999 & -8.9466700 & $(111) /[\overline{1} \overline{1} 2]$ \\
\hline & 0.3373263 & 52.401915 & 27.112499 & -7.6685745 & $(\overline{1} \overline{1} 1) /[\overline{2} 1 \overline{1}]$ \\
\hline \multirow[t]{2}{*}{4.392} & 0.2748234 & 53.680010 & 43.379999 & -8.9466700 & $(111) /[\overline{1} 12]$ \\
\hline & 0.3523419 & 52.401915 & 27.112499 & -7.6685745 & $(\overline{1} 11) /[\overline{2} 1 \overline{1}]$ \\
\hline \multirow[t]{2}{*}{4.3935} & 0.2912923 & 54.958106 & 45.187499 & -7.6685745 & $(111) /[\overline{1} 12]$ \\
\hline & 0.3522622 & 49.845724 & 27.112499 & -7.6685745 & $(\overline{1} 11) /[\overline{2} 1 \overline{1}]$ \\
\hline \multirow[t]{2}{*}{4.395} & 0.2998688 & 54.958106 & 45.187499 & -7.6685745 & $(111) /[\overline{1} 12]$ \\
\hline & 0.3696040 & 51.123819 & 25.304999 & -8.9466700 & $(\overline{1} 11) /[\overline{2} 1 \overline{1}]$ \\
\hline \multirow[t]{2}{*}{4.3965} & 0.3216394 & 52.401915 & 48.802499 & -7.6685745 & $(111) /[112]$ \\
\hline & 0.4011759 & 52.401915 & 23.497499 & -7.6685745 & $(\overline{1} \overline{1} 1) /[\overline{2} 1 \overline{1}]$ \\
\hline \multirow[t]{2}{*}{4.398} & 0.3559017 & 49.845724 & 48.802499 & -7.6685745 & $(111) /[\overline{1} 12]$ \\
\hline & 0.4321144 & 52.401915 & 23.497499 & -7.6685745 & $(\overline{1} \overline{1} 1) /[\overline{2} 1 \overline{1}]$ \\
\hline \multirow[t]{2}{*}{4.3995} & 0.3915396 & 49.845724 & 48.802499 & -7.6685745 & $(111) /[112]$ \\
\hline & 0.4494800 & 52.401915 & 23.497499 & -7.6685745 & $(\overline{1} 11) /[\overline{2} 1 \overline{1}]$ \\
\hline \multirow[t]{2}{*}{4.401} & 0.4162981 & 39.620960 & 37.957499 & -7.6685745 & $(111) /[112]$ \\
\hline & 0.4509573 & 52.401915 & 23.497499 & -7.6685745 & $(\overline{1} 11) /[\overline{2} 1 \overline{1}]$ \\
\hline \multirow[t]{2}{*}{4.4025} & 0.4406552 & 39.620960 & 37.957499 & -7.6685745 & $(111) /[\overline{1} 12]$ \\
\hline & 0.4358870 & 52.401915 & 23.497499 & -7.6685745 & $(\overline{1} 11) /[\overline{2} 1 \overline{1}]$ \\
\hline \multirow[t]{2}{*}{4.404} & 0.4182670 & 39.620960 & 37.957499 & -7.6685745 & $(111) /[1 \overline{1} 12]$ \\
\hline & 0.4288349 & 49.845724 & 23.497499 & -7.6685745 & $(\overline{1} 11) /[\overline{2} 1 \overline{1}]$ \\
\hline \multirow[t]{2}{*}{4.4055} & 0.4352995 & 47.289533 & 48.802499 & -7.6685745 & $(111) /[\overline{1} \overline{1} 2]$ \\
\hline & 0.4362736 & 51.123819 & 21.689999 & -8.9466700 & $(\overline{1} \overline{1} 1) /[\overline{2} 1 \overline{1}]$ \\
\hline \multirow[t]{2}{*}{4.407} & 0.4796819 & 37.064769 & 37.957499 & -7.6685745 & $(111) /[\overline{1} \overline{1} 2]$ \\
\hline & 0.4414554 & 51.123819 & 21.689999 & -8.9466700 & $(\overline{1} 11) /[\overline{2} 1 \overline{1}]$ \\
\hline \multirow[t]{2}{*}{4.4085} & 0.4884835 & 37.064769 & 37.957499 & -7.6685745 & $(111) /[\overline{1} 12]$ \\
\hline & 0.4501391 & 39.620960 & 27.112499 & -7.6685745 & $(\overline{1} \overline{1} 1) /[\overline{2} 1 \overline{1}]$ \\
\hline \multirow[t]{2}{*}{4.41} & 0.4655257 & 37.064769 & 37.957499 & -7.6685745 & $(111) /[112]$ \\
\hline & 0.4835746 & 39.620960 & 27.112499 & -7.6685745 & $(\overline{1} 11) /[\overline{2} 11]$ \\
\hline
\end{tabular}

Table 5.2: The predicted nucleation sites and slip system of dislocation with the maximum $\mathrm{N}_{m, l}$ value for $\mathrm{Cu}(110)$ model. These results agree with the actual nucleation sites shown in Figure 5.8, and slip system $(111) / \frac{1}{6}[\overline{1} \overline{1} 2]$ and $(\overline{1} 11) / \frac{1}{6}[\overline{2} 1 \overline{1}]$.

101 


\section{Ni (110) model}

Figure 5.9 shows the indenter load versus displacement curve and the $\mathrm{N}_{m, l}$ versus displacement curve for the Ni (110) model. Since the influence of material hardness, the drop of indenter load is found at a displacement of $5.105 \AA$, which is different with the $\mathrm{Cu}(110)$ (i.e., $4.386 \AA$ ). The $\mathrm{N}_{\text {crit }}$ is approximately $0.20 \pm 0.02\left(\mathrm{eV} / \AA^{4}\right)$, which is close to the $\mathrm{N}_{\text {crit }}$ value of the $\mathrm{Ni}(001)$ (i.e., $0.21 \pm 0.02\left(\mathrm{eV} / \stackrel{\circ}{A}^{4}\right)$ ) and different with the $\mathrm{Cu}(110)$ (i.e., $0.15 \pm 0.02\left(\mathrm{eV} / \AA^{4}\right)$ ), therefore, the fact is proved again that the $\mathrm{N}_{\text {crit }}$ is a material related property and does not depend on the crystalline orientations.

Figure 5.10 shows the comparison between the actual dislocation nucleation and the $\mathrm{N}_{m, l}$ predictions for the $\mathrm{Ni}(110)$ model. The predicted dislocations are found to be Shockley partial dislocations $(111) / \frac{1}{6}[\overline{1} 12]$ and $\left.(\overline{1} 11) / \frac{1}{6}[\overline{2} 1 \overline{1}]\right)$, which are same as the actual dislocations prdicted by the slip vector. The configuration of dislocation structure is similar as the $\mathrm{Cu}(110)$ because of the same crystalline orientation.

Table 5.3 shows the numerical prediction results using the "Acharya/Miller" criterion for the Ni (110) model. All these values are collected prior to the nucleation event from the moment of $5.045 \AA$ depth, the maximum $\mathrm{N}_{m, l}$ value occurs at the same location as the actual site and the slip system are Shockley partials (111)/ $\frac{1}{6}[\overline{1} 12]$ and $(\overline{1} \overline{1} 1) / \frac{1}{6}[\overline{2} 1 \overline{1}]$, which are same as the actual dislocations indicated in Figure 5.10. Comparing with table 5.2, although the depth of nucleation sites are different since the material properties, the configuration of dislocation structure for $\mathrm{Cu}(110)$ and $\mathrm{Ni}(110)$ are same because of the same crystalline orientation. 

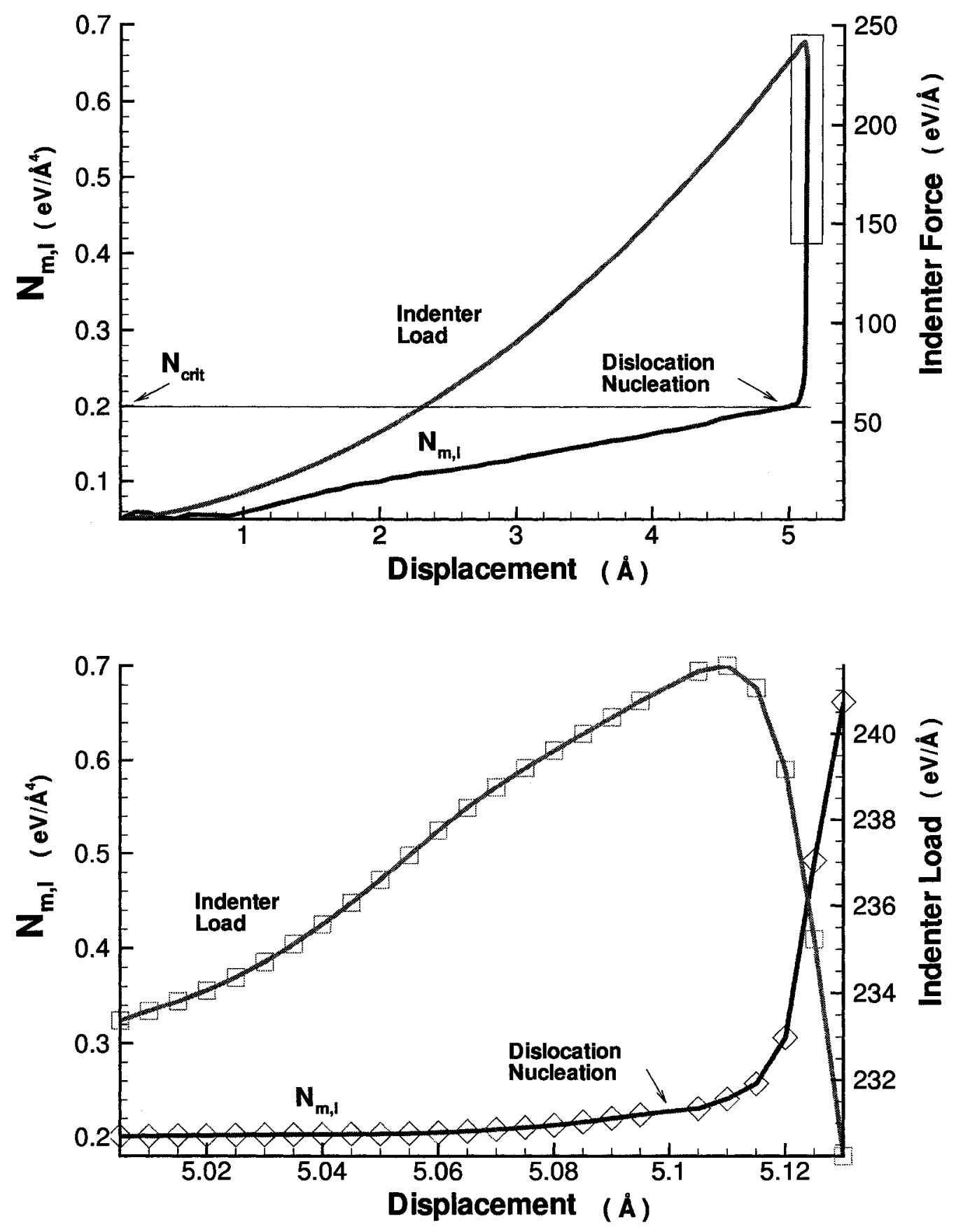

Figure 5.9: The correlation of two curves: the indenter load versus displacement curve and the maximum $\mathrm{N}_{m, l}$ versus displacement curves for the $\mathrm{Ni}$ (110) model. The lower figure is an enlargement of the late stages of the top graphs. 


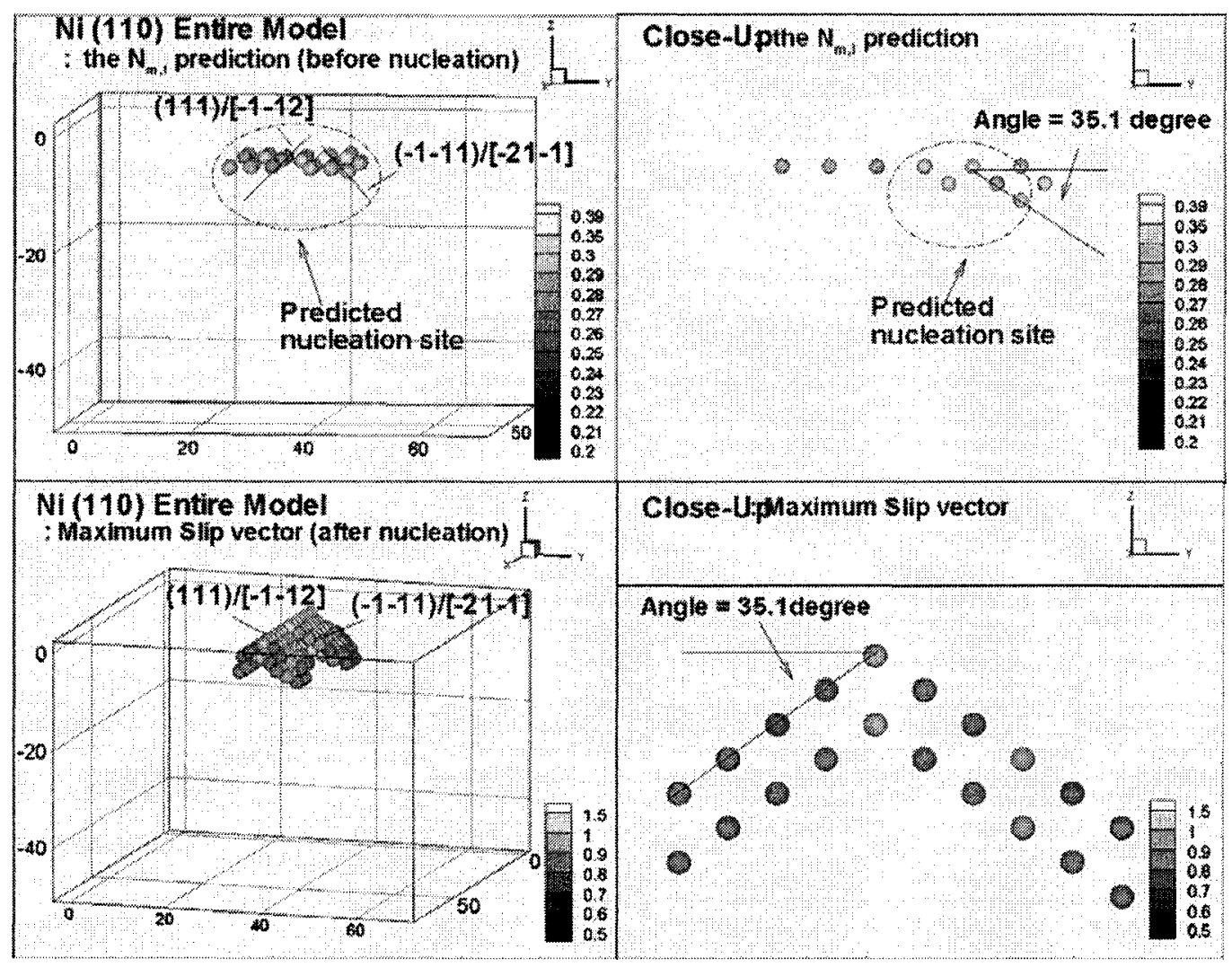

Figure 5.10: The comparison of $\mathrm{N}_{m, l}$ prediction and actual dislocations are shown for the Ni (110) model. The bottom part of the figure is the slip vector distribution immediately after the nucleation event, and the top part of the figure is the maximum $\mathrm{N}_{m, l}$ distribution throughout the atom slab. 


\begin{tabular}{|c|c|c|c|c|c|}
\hline \multirow{2}{*}{ Depth } & \multirow{2}{*}{$\operatorname{Max} \mathrm{N}_{m, l}$} & \multicolumn{4}{|c|}{ Predicted nucleation site and dislocation character } \\
\hline & & Coor $\_x$ & Coor_y & Coor_z & Slip system \\
\hline \multirow[t]{2}{*}{5.045} & 0.2034800 & 22.40114 & 28.16000 & -6.22253 & $(111) /[\overline{1} \overline{1} 2]$ \\
\hline & 0.2022408 & 52.26933 & 42.24000 & -6.22253 & $(\overline{1} 11) /[112]$ \\
\hline \multirow[t]{2}{*}{5.05} & 0.2035968 & 22.40114 & 28.16000 & -6.22253 & $(111) /[\overline{1} 12]$ \\
\hline & 0.2032450 & 52.26933 & 42.24000 & -6.22253 & $(\overline{1} 11) /[112]$ \\
\hline \multirow[t]{2}{*}{5.055} & 0.2042684 & 22.40114 & 28.16000 & -6.22253 & $(111) /[\overline{1} \overline{1} 2]$ \\
\hline & 0.2037665 & 52.26933 & 42.24000 & -6.22253 & $(\overline{1} 11) /[112]$ \\
\hline \multirow[t]{2}{*}{5.06} & 0.2050418 & 22.40114 & 28.16000 & -6.22253 & $(111) /[112]$ \\
\hline & 0.2049909 & 52.26933 & 42.24000 & -6.22253 & $(\overline{1} 11) /[112]$ \\
\hline \multirow[t]{2}{*}{5.065} & $0 . \overline{2061451}$ & 22.40114 & 28.16000 & -6.22253 & $(111) /[\overline{1} 12]$ \\
\hline & 0.2063804 & 52.26933 & 42.24000 & -6.22253 & $(\overline{1} 11) /[112]$ \\
\hline \multirow[t]{2}{*}{5.07} & 0.2075831 & 22.40114 & 28.16000 & -6.22253 & $(111) /[112]$ \\
\hline & 0.2082601 & 52.26933 & 42.24000 & -6.22253 & $(\overline{1} 11) /[112]$ \\
\hline \multirow[t]{2}{*}{5.075} & 0.2092076 & 22.40114 & 28.16000 & -6.22253 & $(111) /[112]$ \\
\hline & 0.2103484 & 52.26933 & 42.24000 & -6.22253 & $(\overline{1} 11) /[112]$ \\
\hline \multirow[t]{2}{*}{5.08} & 0.2121376 & 22.40114 & 28.16000 & -6.22253 & $(111) /[112]$ \\
\hline & 0.2127514 & 52.26933 & 42.24000 & -6.22253 & $(111) /[112]$ \\
\hline \multirow[t]{2}{*}{5.085} & 0.2141633 & 22.40114 & 28.16000 & -6.22253 & $(111) /[\overline{1} 12]$ \\
\hline & 0.2162415 & 52.26933 & 42.24000 & -6.22253 & $(\overline{1} 11) /[112]$ \\
\hline \multirow[t]{2}{*}{5.09} & 0.2172391 & 22.40114 & 28.16000 & -6.22253 & $(111) /[112]$ \\
\hline & 0.2201356 & 52.26933 & 42.24000 & -6.22253 & $(\overline{1} 11) /[112]$ \\
\hline \multirow[t]{2}{*}{5.095} & 0.2207840 & 22.40114 & 28.16000 & -6.22253 & $(111) /[112]$ \\
\hline & 0.2237800 & 52.26933 & 42.24000 & -6.22253 & $(\overline{1} 11) /[112]$ \\
\hline \multirow[t]{2}{*}{5.105} & 0.2253231 & 22.40114 & 28.16000 & -6.22253 & $(111) /[112]$ \\
\hline & 0.2306970 & 52.26933 & 42.24000 & -6.22253 & $(\overline{1} 11) /[112]$ \\
\hline \multirow[t]{2}{*}{5.11} & 0.2318279 & 22.40114 & 28.16000 & -6.22253 & $(111) /[\overline{1} 12]$ \\
\hline & 0.2409520 & 52.26933 & 42.24000 & -6.22253 & $(\overline{1} 11) /[112]$ \\
\hline \multirow[t]{2}{*}{5.115} & 0.2439217 & 22.40114 & 28.16000 & -6.22253 & $(111) /[\overline{1} 12]$ \\
\hline & 0.2573080 & 52.26933 & 42.24000 & -6.22253 & $(\overline{1} 11) /[112]$ \\
\hline \multirow[t]{2}{*}{5.12} & 0.2849388 & 22.40114 & 28.16000 & -6.22253 & $(111) /[1112]$ \\
\hline & 0.2914625 & 52.26933 & 42.24000 & -6.22253 & $(\overline{1} \overline{1} 1) /[112]$ \\
\hline
\end{tabular}

Table 5.3: The predicted nucleation sites and slip systems of dislocations with the maximum $\mathrm{N}_{m, l}$ value for the $\mathrm{Ni}(110)$ model. These results agree with the actual nucleation sites shown in Figure 5.10, and slip systems $(111) / \frac{1}{6}[\overline{1} \overline{1} 2]$ and $(\overline{1} \overline{1} 1) / \frac{1}{6}[\overline{2} 1 \overline{1}]$. 


\section{Cu (111) model}

Figure 5.11 illustrates a snapshot of the nucleation presented by the slip vector in a side view (a rotation between $[1 \overline{1} 0]$ and $[11 \overline{2}]$ directions) near the first plastic yield point for the $\mathrm{Cu}$ (111) model. It is obvious that two similar size of embryos nucleate on the same inclined (111) slip plane below the indentation surface and inside the contact area simultaneously, and the site and slip mode of these two embryos are symmetric with respect to $z$ axis. Embryo size is the indication of the sequence of embryo nucleation, with larger embryo nucleating at earlier moment. Afterward, the embryos is found to expand into two partial dislocation loops, and then extend into the dislocation loops.

Figure 5.12 shows the contour of the slip vector in the same view as Figure 5.11 at the moment of $5.585 \AA$ indenter depth after the nucleation event. It is obvious that the two Shockley partial dislocations (i.e., $(\overline{1} \overline{1} 1) / \frac{1}{6}[1 \overline{2} \overline{1}]$ and $\left.(\overline{1} \overline{1} 1) / \frac{1}{6}[\overline{2} 1 \overline{1}]\right)$ glide and intersect with each other to form an inclined plane dislocation structure. It is also obvious that the glide process of dislocations always occurs on one of the three inclined $\{111\}$ type of slip planes at the very early stage. A three fold symmetric defect structure is never obtained, the reason why dislocation structures loss the $\{111\}$ surface three fold symmetry can be understood that the two nucleated dislocations with symmetric sites and slip modes could occupy geometrically the deformation imposed by the indenter penetration and the subsequent activation of the glide process is possibly to be constrained for a while.

Figure 5.13 shows the distribution of the slip vector on the (1111) slip plane for the $\mathrm{Cu}$ (111) model, and each Shockley partial bounding with a stacking fault. The maximum magnitude of slip vector is still around $1.47 \AA$ for $\mathrm{Cu}$ and $1.66 \AA$ for $\mathrm{Ni}$.

Figure 5.14 shows the front view (along the [110] direction) of the same dislocation 
structure, it is obvious that the slip vectors along two layers of atoms with the opposite slip directions.

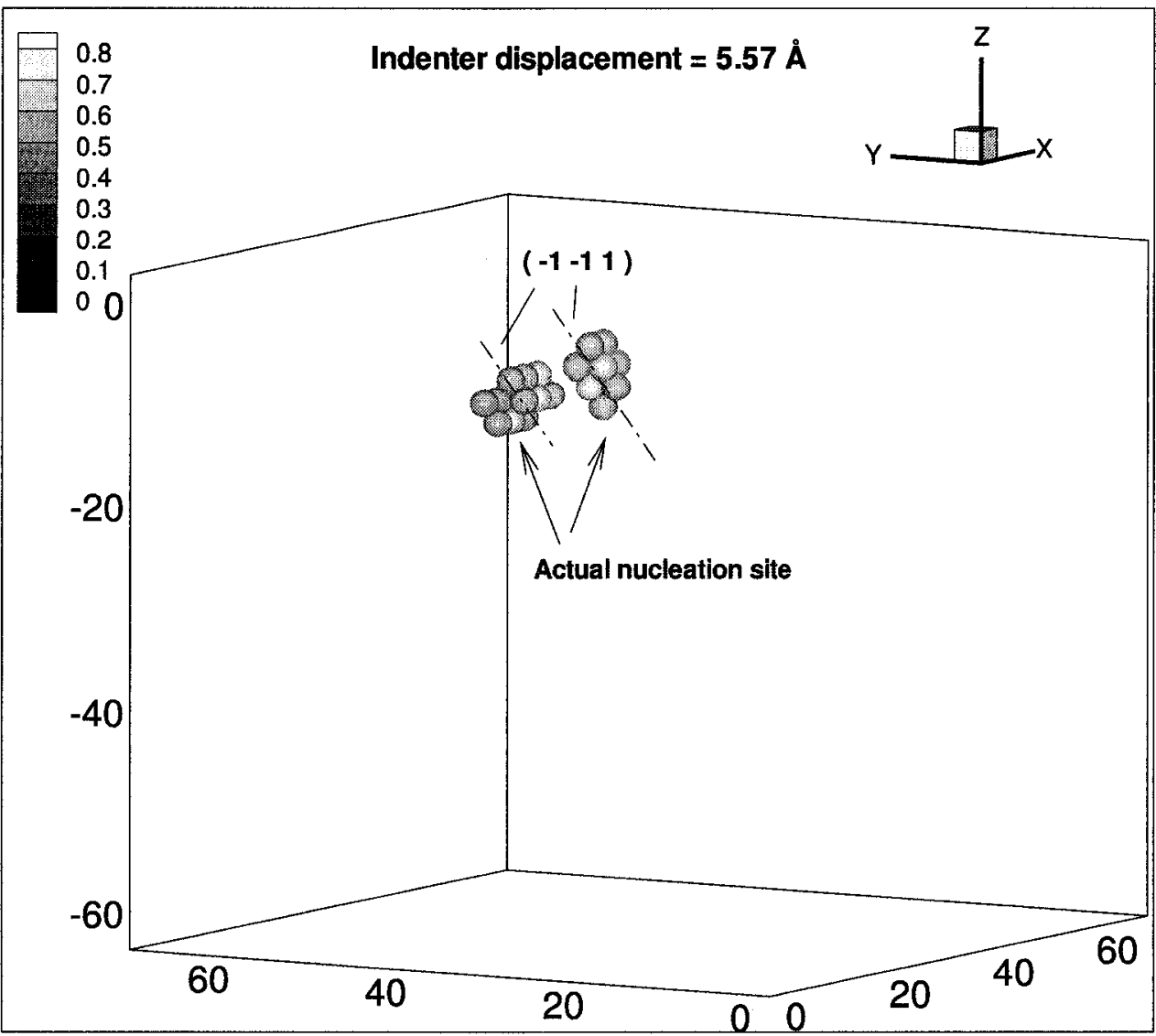

Figure 5.11: The snapshot of dislocation nucleation is presented by the slip vector for the $\mathrm{Cu}(111)$ model. 


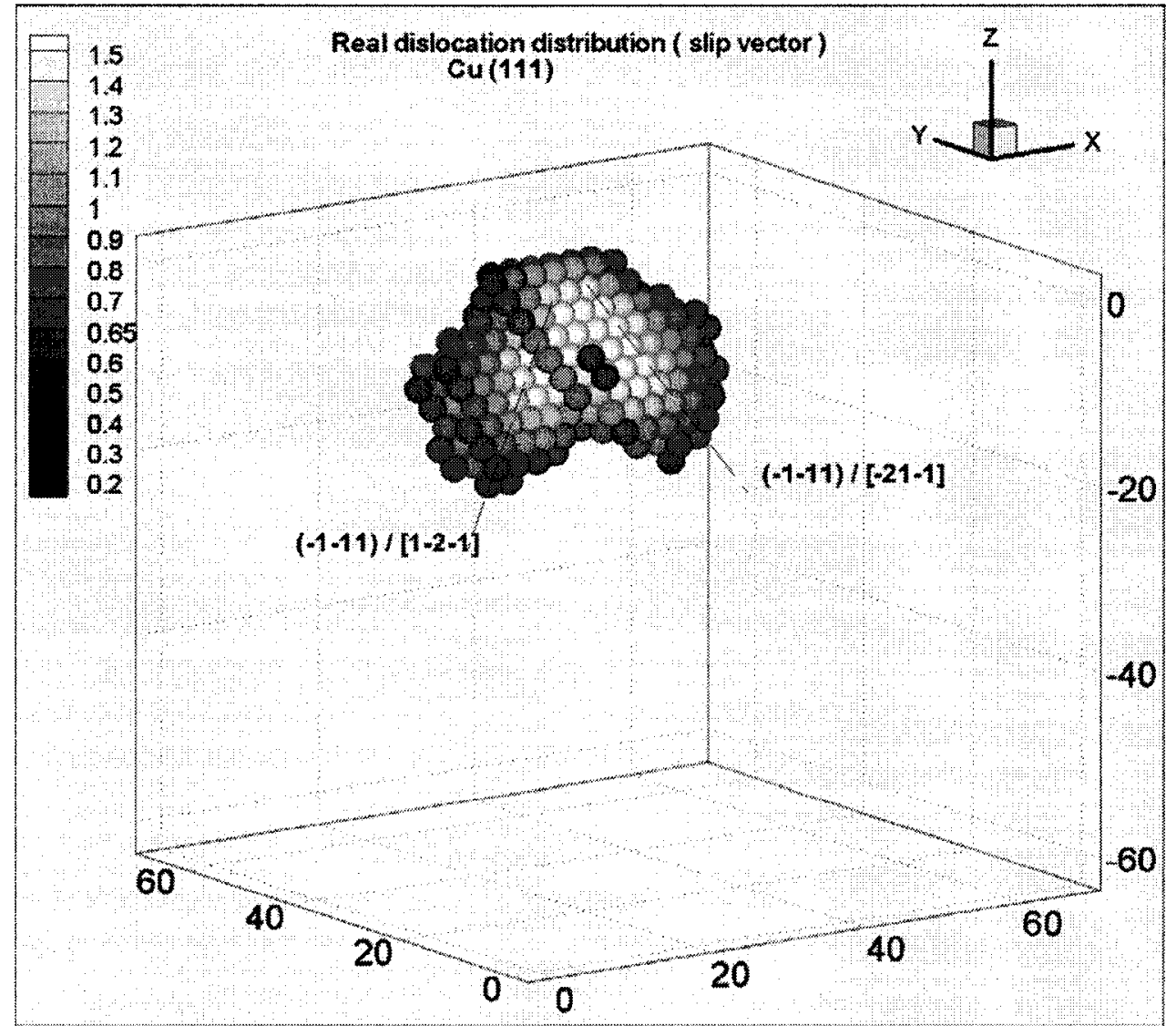

Figure 5.12: Contour of the slip vector on a slip plane to demonstrate the actual dislocation structure beneath the indentation (111) surface. 


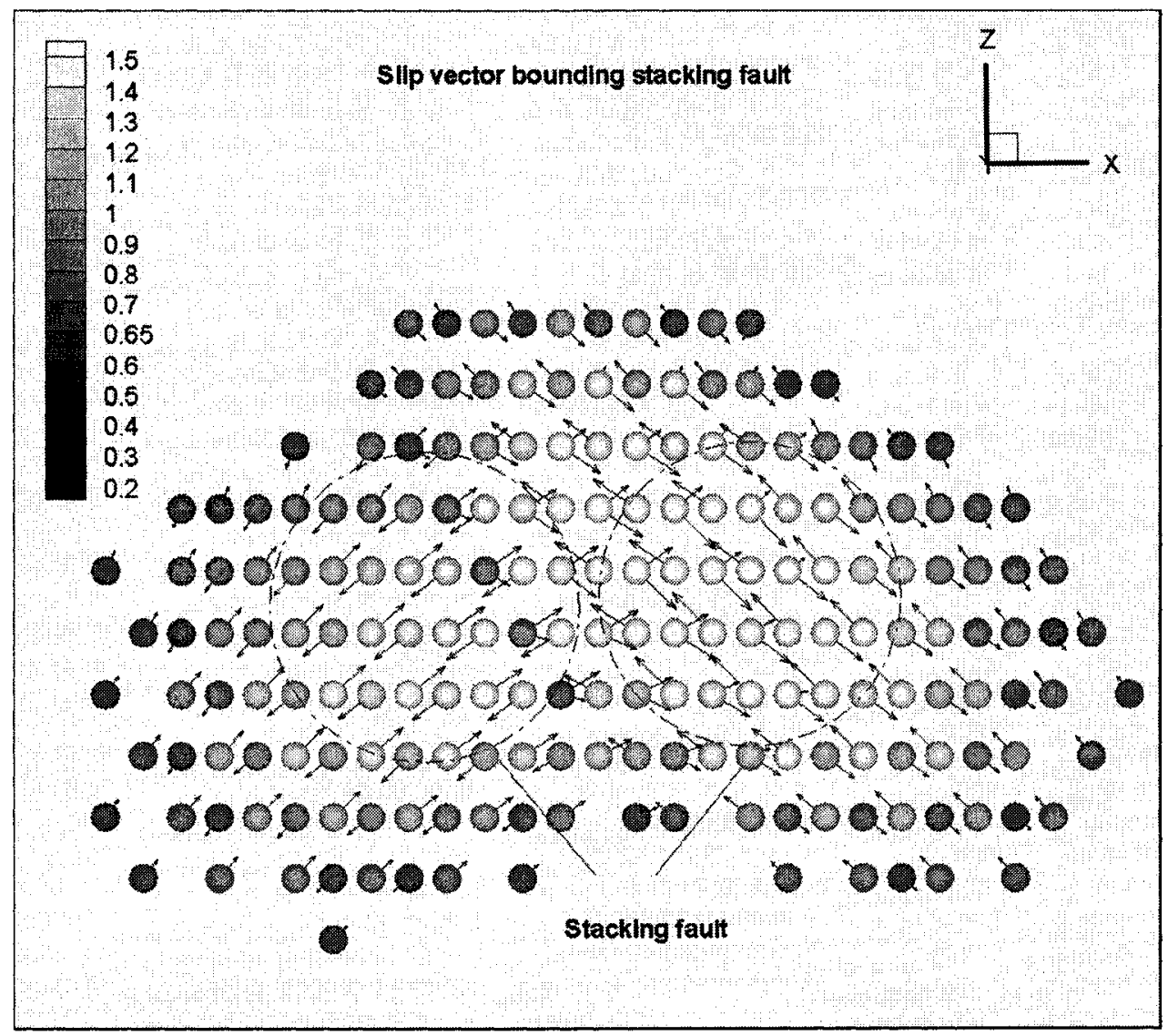

Figure 5.13: Vector graph of slip vector on a slip plane to demonstrate the magnitude and direction of Burgers vector for the $\mathrm{Cu}$ (111) model. 


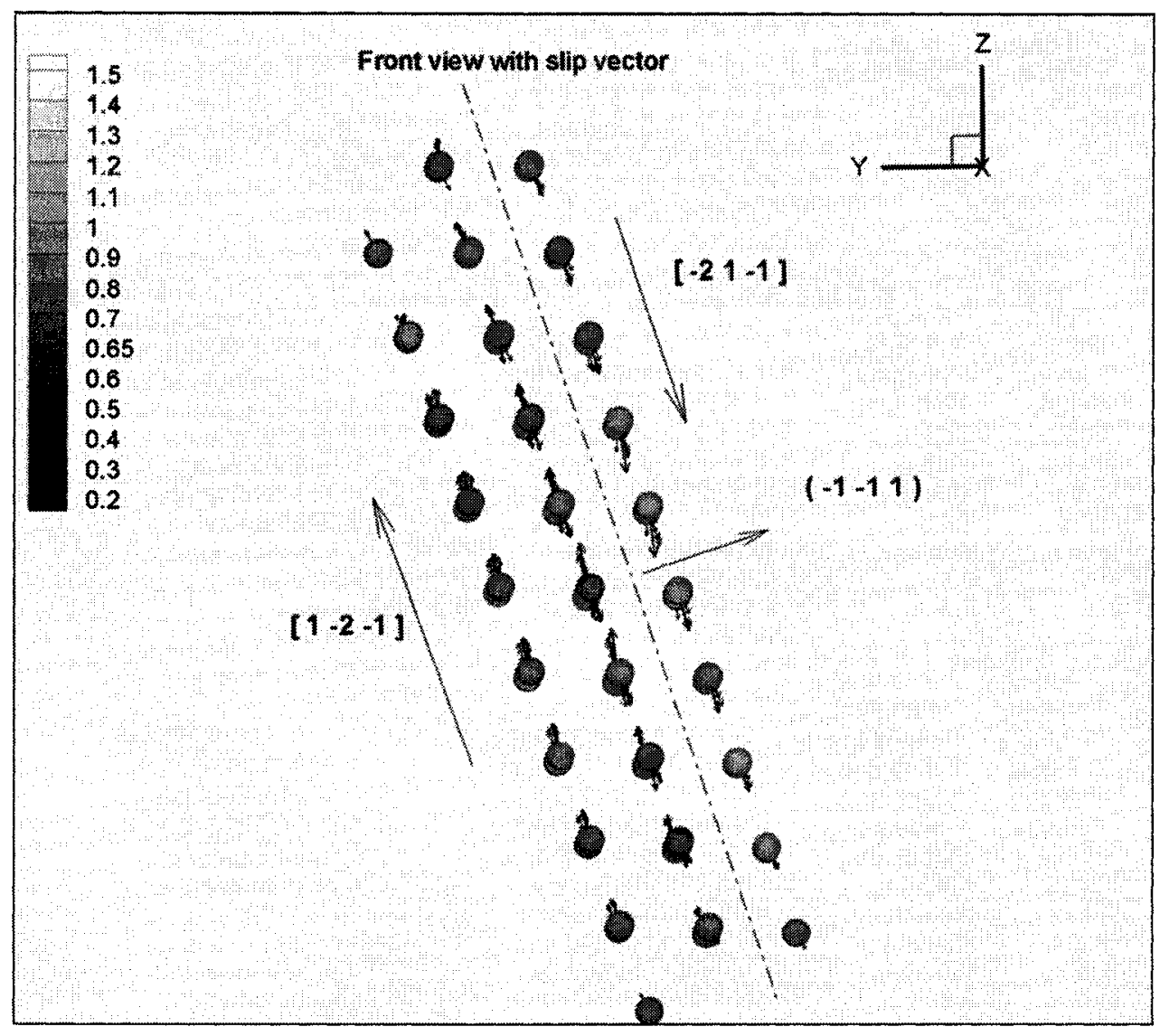

Figure 5.14: Front view of actual dislocation structure to demonstrate the magnitude and direction of Burgers vector for the $\mathrm{Cu}$ (111) model. 

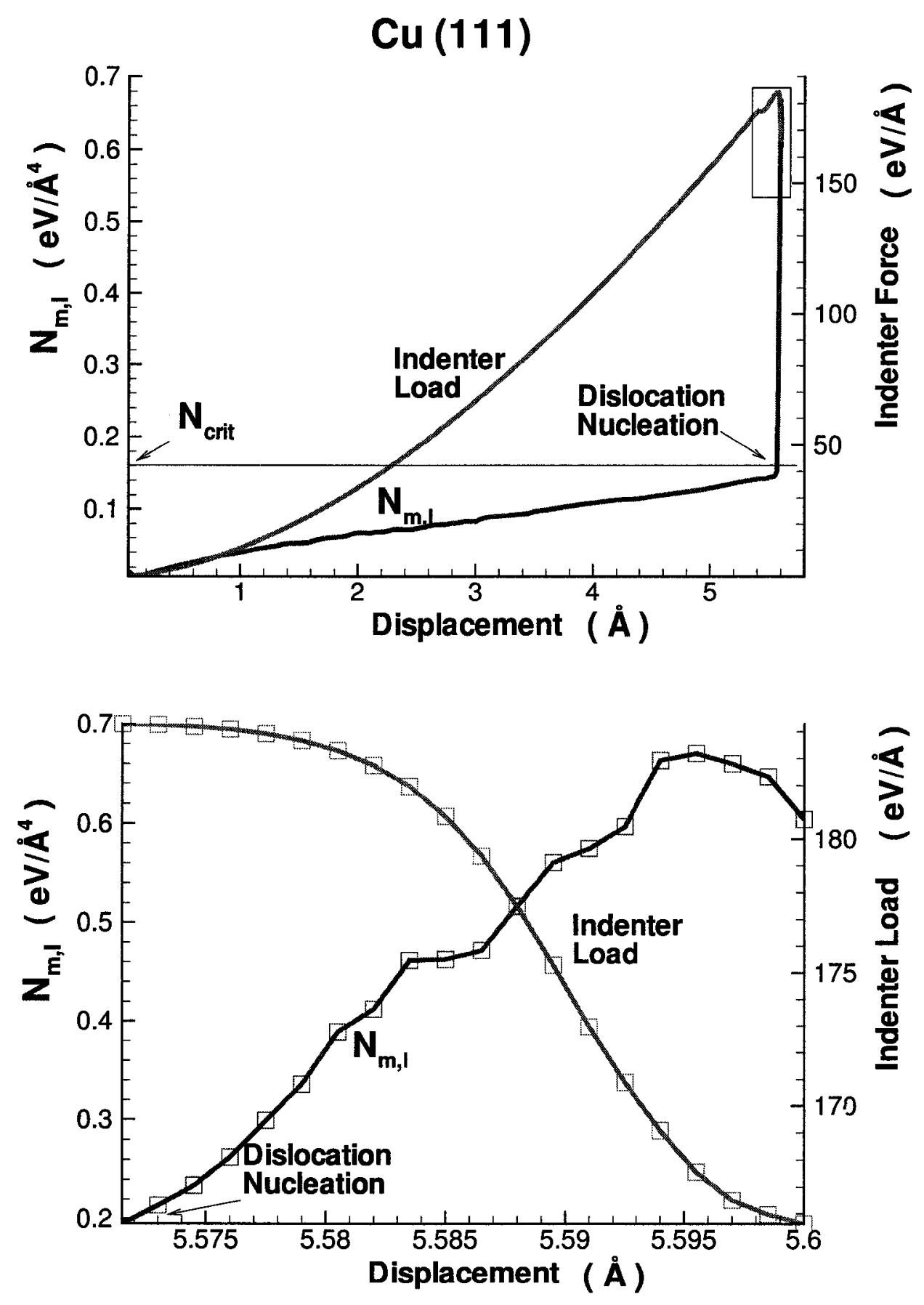

Figure 5.15: The correlation of two curves: the indenter load versus displacement curve and the maximum $\mathrm{N}_{m, l}$ versus displacement curve for the $\mathrm{Cu}$ (111) model. The lower figure is an enlargement of the late stages of the top graphs. 
Figure 5.15 shows the indenter load versus displacement curve and the $\mathrm{N}_{m, l}$ versus displacement curve for the $\mathrm{Cu}(111)$ model. similarly, the nucleation event accompanies with a abrupt drop of indenter load and a sudden jump of the $\mathrm{N}_{m, l}$ value. Specifically, the indenter load drops are found at an indentation depth of $5.57 \AA$ when the "Acharya/Miller" criterion first detects the nucleation. The $\mathrm{N}_{\text {crit }}$ value is about $0.16 \pm 0.02\left(\mathrm{eV} / \AA^{4}\right)$ for the $\mathrm{Cu}(111)$ model, it is almost the same $\mathrm{N}_{\text {crit }}$ value for the $\mathrm{Cu}(110)\left(0.15 \pm 0.02\left(\mathrm{eV} / \AA^{4}\right)\right)$ and $\mathrm{Cu}(001)\left(0.16 \pm 0.02\left(\mathrm{eV} / \AA^{4}\right)\right)$, but much different with the $\mathrm{Ni}(001)\left(0.21 \pm 0.02\left(\mathrm{eV} / \stackrel{\circ}{A}^{4}\right)\right)$ and $\mathrm{Ni}(110)\left(0.22 \pm 0.02\left(\mathrm{eV} / \AA^{4}\right)\right)$. These facts again prove that the $\mathrm{N}_{\text {crit }}$ value is a material related property and does not depend on crystal orientations.

Figure 5.16 is the snapshots comparison of the actual dislocations indicated by the slip vector and the $\mathrm{N}_{m, l}$ predictions for the $\mathrm{Cu}(111)$ model. The predicted nucleation site agrees with the actual site, and the predicted shockley partials are $(\overline{1} \overline{1} 1) / \frac{1}{6}[1 \overline{2} \overline{1}]$ and $(\overline{1} \overline{1} 1) / \frac{1}{6}[\overline{2} 1 \overline{1}]$, which are same as the actual dislocations. The capability of "Acharya/Miller" criterion is examined again to predict when and where nucleations occur.

Table 5.4 shows the numerical prediction result for the $\mathrm{Cu}$ (111) model using the "Acharya/Miller" criterion. All these values are derived prior to the nucleation event from the moment of $5.582 \AA$ depth, the predicted nucleation sites and slip system (i.e., $(\overline{1} \overline{1} 1) / \frac{1}{6}[1 \overline{2} \overline{1}]$ and $\left.(\overline{1} \overline{1} 1) / \frac{1}{6}[\overline{2} 1 \overline{1}]\right)$ agrees with the actual results. This table demonstrates that "Acharya/Miller" criterion is an efficient predictor for this model. 


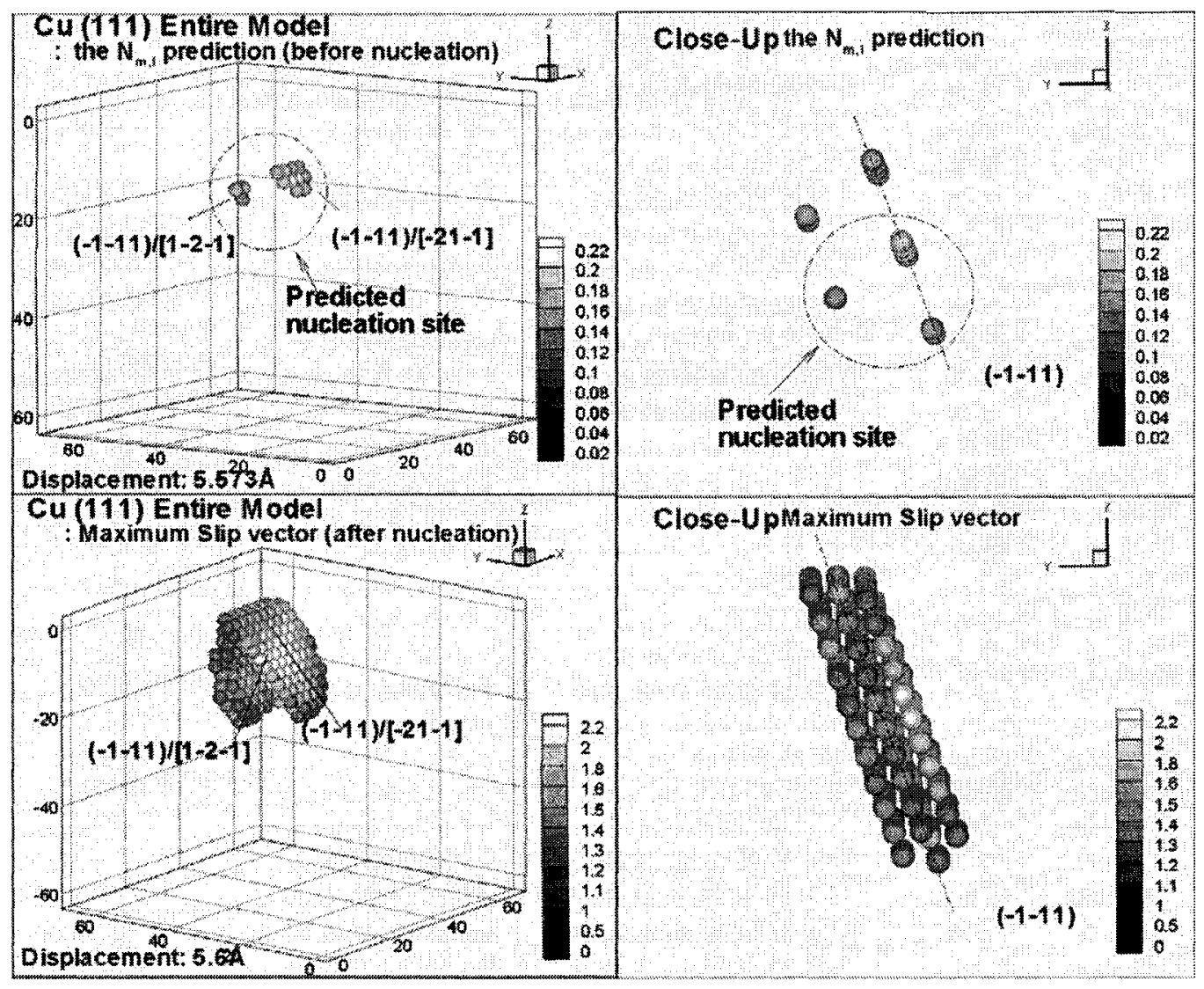

Figure 5.16: The comparison of prediction result and actual dislocation is shown for the $\mathrm{Cu}$ (111) model. The bottom part of the figure is the slip vector distribution immediately after the nucleation event, and top part of the figure is the maximum $\mathrm{N}_{m, l}$ value distribution throughout the atom slab. 


\begin{tabular}{|c|c|c|c|c|c|}
\hline \multirow{2}{*}{ Depth } & \multirow{2}{*}{$\operatorname{Max} \mathrm{N}_{m, l}$} & \multicolumn{4}{|c|}{ Predicted nucleation site and dislocation character } \\
\hline & & Coor_x & Coor_y & Coor_z & Slip system \\
\hline \multirow[t]{2}{*}{5.582} & 0.4018244 & 35.78667 & 42.79870 & -8.34848 & $(\overline{1} \overline{1} 1) /[\overline{2} 1 \overline{1}]$ \\
\hline & 0.3839969 & 42.17715 & 36.15753 & -14.6098 & $(\overline{1} \overline{1} 1) /[1 \overline{2} \overline{1}]$ \\
\hline \multirow[t]{3}{*}{5.5835} & 0.4616884 & 43.45524 & 35.41962 & -16.6969 & $(\overline{1} 11) /[1 \overline{2} \overline{1}]$ \\
\hline & 0.4177828 & 35.78667 & 42.79870 & -8.34848 & $(\overline{1} \overline{1} 1) /[\overline{2} 1 \overline{1}]$ \\
\hline & 0.4161719 & 33.23048 & 36.89543 & -12.5227 & $(\overline{1} 11) /[112]$ \\
\hline \multirow[t]{2}{*}{5.585} & 0.4627344 & 43.45524 & 35.41962 & -16.6969 & $(\overline{1} 11) /[12 \overline{1}]$ \\
\hline & 0.4177828 & 35.78667 & 42.79870 & -8.34848 & $(\overline{1} \overline{1} 1) /[\overline{2} 1 \overline{1}]$ \\
\hline \multirow[t]{3}{*}{5.5865} & 0.4714652 & 43.45524 & 35.41962 & -16.6969 & $(\overline{1} \overline{1} 1) /[1 \overline{2} \overline{1}]$ \\
\hline & 0.4507268 & 34.50857 & 37.63334 & -16.6969 & $(\overline{1} \overline{1} 1) /[112]$ \\
\hline & 0.4041384 & 20.44952 & 36.89543 & -18.7840 & $(\overline{1} \overline{1} 1) /[\overline{2} 1 \overline{1}]$ \\
\hline \multirow[t]{3}{*}{5.588} & 0.5166430 & 34.50857 & 37.63334 & -16.6969 & $(\overline{111}) /[112]$ \\
\hline & 0.4884715 & 42.17715 & 34.68171 & -18.7840 & $(\overline{1} \overline{1} 1) /[1 \overline{2} \overline{1}]$ \\
\hline & 0.4305914 & 20.44952 & 36.89543 & -18.7840 & $(\overline{1} \overline{1} 1) /[\overline{2} 1 \overline{1}]$ \\
\hline \multirow[t]{3}{*}{5.5895} & 0.5601816 & 34.50857 & 37.63334 & -16.6969 & $(\overline{1} 11) /[112]$ \\
\hline & 0.4892944 & 44.73334 & 34.68171 & -18.7840 & $(\overline{1} \overline{1} 1) /[1 \overline{2} \overline{1}]$ \\
\hline & 0.4335863 & 17.89333 & 36.89543 & -18.7840 & $(\overline{1} 11) /[\overline{2} 1 \overline{1}]$ \\
\hline \multirow[t]{3}{*}{5.591} & 0.5743296 & 34.50857 & 37.63334 & -16.6969 & $(\overline{1} 1) /[112]$ \\
\hline & 0.4967916 & 43.45524 & 33.94380 & -20.8712 & $(\overline{1} \overline{1} 1) /[1 \overline{2} \overline{1}]$ \\
\hline & 0.4482801 & 19.17143 & 36.15753 & -20.8712 & $(\overline{1} 11) /[\overline{2} 1 \overline{1}]$ \\
\hline \multirow[t]{3}{*}{5.5925} & 0.5966451 & 33.23048 & 36.89543 & -18.7840 & $(\overline{1} 11) /[112]$ \\
\hline & 0.4947319 & 43.45524 & 33.94380 & -20.8712 & $(\overline{1} \overline{1} 1) /[1 \overline{2} \overline{1}]$ \\
\hline & 0.4488915 & 19.17143 & 36.15753 & -20.8712 & $(\overline{1} \overline{1} 1) /[\overline{2} 1 \overline{1}]$ \\
\hline \multirow[t]{2}{*}{5.594} & 0.6633098 & 33.23048 & 36.89543 & -18.7840 & $(\overline{1} 1) /[112]$ \\
\hline & 0.4846116 & 38.34286 & 33.94380 & -20.8712 & $(\overline{1} \overline{1} 1) /[1 \overline{2} \overline{1}]$ \\
\hline \multirow[t]{2}{*}{5.5955} & 0.6701863 & 33.23048 & 36.89543 & -18.7840 & $(111) /[112]$ \\
\hline & 0.4803872 & 42.17715 & 33.20589 & -22.9583 & $(\overline{1} \overline{1} 1) /[1 \overline{2} \overline{1}]$ \\
\hline \multirow[t]{2}{*}{5.597} & 0.6597953 & 33.23048 & 36.89543 & -18.7840 & $(\overline{1} 1) /[112]$ \\
\hline & 0.4791855 & 39.62096 & 33.20589 & -22.9583 & $(\overline{1} \overline{1} 1) /[1 \overline{2} \overline{1}]$ \\
\hline \multirow[t]{2}{*}{5.5985} & 0.6468411 & 33.23048 & 36.89543 & -18.7840 & $(\overline{11} 1) /[112]$ \\
\hline & 0.4697686 & 39.62096 & 33.20589 & -22.9583 & $(\overline{1} \overline{1} 1) /[1 \overline{2} \overline{1}]$ \\
\hline \multirow[t]{2}{*}{5.6} & 0.6039965 & 33.23048 & 36.89543 & -18.7840 & $(111) /[112]$ \\
\hline & 0.4639258 & 37.06476 & 33.20589 & -22.9583 & $(\overline{1} \overline{1} 1) /[1 \overline{2} \overline{1}]$ \\
\hline
\end{tabular}

Table 5.4: The predicted nucleation sites and slip system of dislocation with the maximum $\mathrm{N}_{m, l}$ value for the $\mathrm{Cu}(111)$ model. These results agree with the actual nucleation sites and slip systems $(\overline{1} \overline{1} 1) / \frac{1}{6}[1 \overline{2} \overline{1}]$ and $(\overline{1} \overline{1} 1) / \frac{1}{6}[\overline{2} 1 \overline{1}]$. 


\section{Ni (111) model}

Figure 5.17 shows the indenter load versus displacement curve and the $\mathrm{N}_{m, l}$ versus displacement curve for the $\mathrm{Ni}(111)$ model. Again, the indenter load drops when the $\mathrm{N}_{m, l}$ reaches to the $\mathrm{N}_{c r i t}$. Specifically, the $\mathrm{N}_{\text {crit }}$ is around $0.22 \pm 0.02\left(\mathrm{eV} / \stackrel{\circ}{\mathrm{A}}^{4}\right)$, it is close to the $\mathrm{N}_{\text {crit }}$ value for $\mathrm{Ni}(110)\left(0.22 \pm 0.02\left(\mathrm{eV} / \AA^{4}\right)\right)$ and $\mathrm{Ni}(001)(0.21 \pm$ $\left.0.02\left(\mathrm{eV} / \AA^{4}\right)\right)$ models, but much different with $\mathrm{Cu}(111)\left(0.16 \pm 0.02\left(\mathrm{eV} / \AA^{4}\right)\right)$ and $\mathrm{Cu}(110)\left(0.16 \pm 0.02\left(\mathrm{eV} / \AA^{4}\right)\right)$.

Figure 5.18 is again the visualization of the actual dislocations indicated by the slip vector and the $\mathrm{N}_{m, l}$ predictions for the $\mathrm{Ni}$ (111) model. The predicted results are same as the actual results, and the same shockley partials $(\overline{1} \overline{1} 1) / \frac{1}{6}[1 \overline{2} \overline{1}]$ and $(\overline{1} 11) / \frac{1}{6}[\overline{2} 1 \overline{1}]$ are detected as in the $\mathrm{Cu}(111)$ model, this fact indicates that the crystalline orientation is an important factor to influence the dislocation structure.

Like Table 5.4, Table 5.5 shows the numerical prediction result for the $\mathrm{Ni}$ (111) model using the "Acharya/Miller" criterion. All these values are derived prior to the nucleation event from the moment of $5.805 \AA$ depth, the predicted nucleation sites and slip systems (i.e., $(\overline{1} \overline{1} 1) / \frac{1}{6}[1 \overline{2} \overline{1}]$ and $(\overline{1} \overline{1} 1) / \frac{1}{6}[\overline{2} 1 \overline{1}]$ ) agree with the actual results. This table proves the prediction accuracy of the "Acharya/Miller" criterion for the Ni (111) model. 

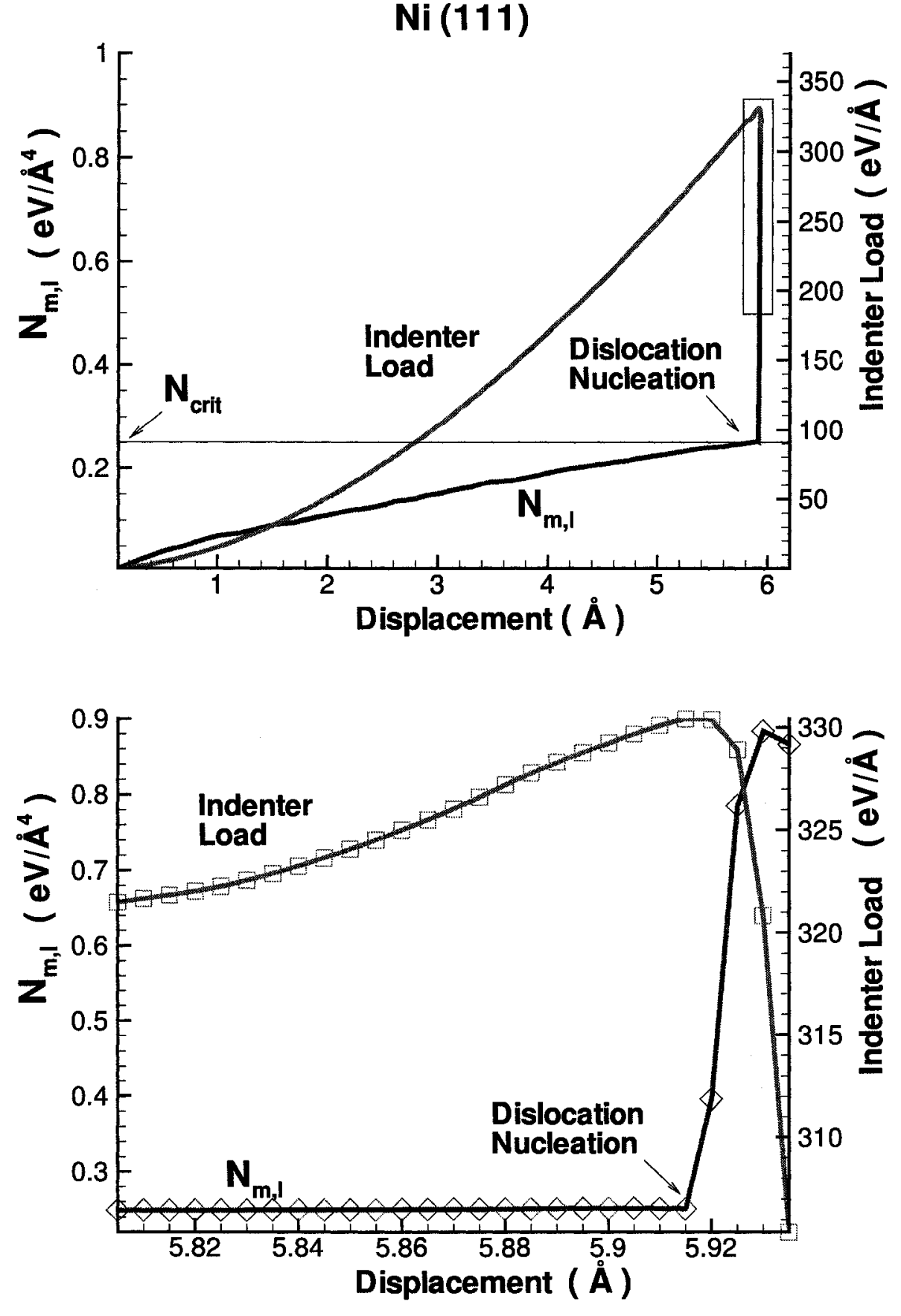

Figure 5.17: The correlation of two curves: the indenter load versus displacement curve and the maximum $\mathrm{N}_{m, l}$ versus displacement curves for the $\mathrm{Ni}$ (111) model. The lower figure is an enlargement of the late stages of the top graphs. 


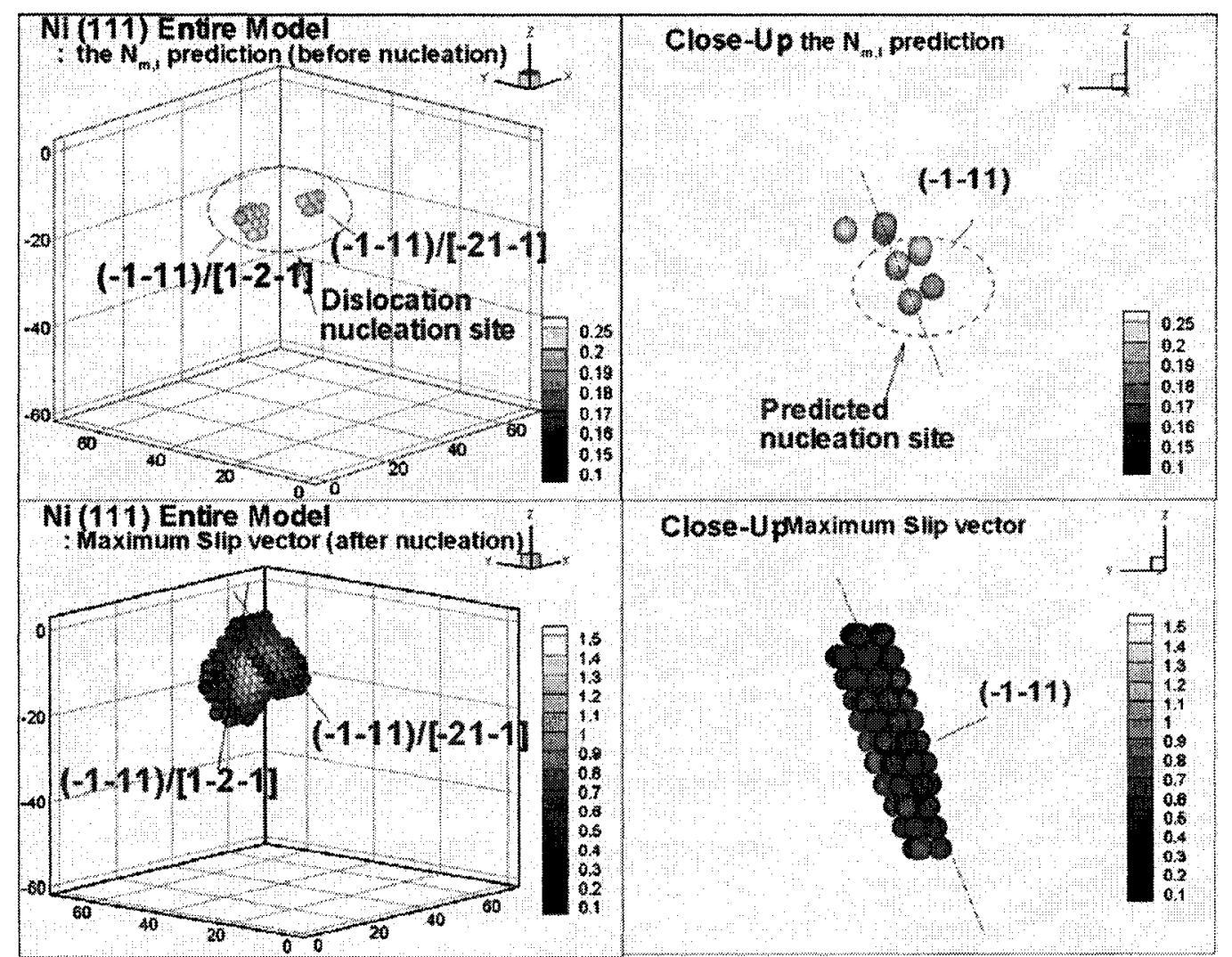

Figure 5.18: The comparison of prediction result and actual dislocation are shown for the Ni (111) model. The bottom part of the figure is the slip vector distribution immediately after the nucleation event, and top part of the figure is the maximum $\mathrm{N}_{m, l}$ value distribution throughout the atom slab. 


\begin{tabular}{|c|c|c|c|c|c|}
\hline \multirow{2}{*}{ Depth } & \multirow{2}{*}{$\operatorname{Max} \mathrm{N}_{m, l}$} & \multicolumn{4}{|c|}{ Predicted nucleation site and dislocation character } \\
\hline & & Coor_x & Coor_y & Coor_z & Slip system \\
\hline 5.805 & 0.2488181 & 34.8462 & 53.170257 & -6.09682 & $(\overline{1} 11) /[112]$ \\
\hline 5.81 & 0.2488407 & 34.8462 & 53.170257 & -6.09682 & $(\overline{1} \overline{1} 1) /[112]$ \\
\hline 5.815 & 0.2490234 & 34.8462 & 53.170257 & -6.09682 & $(111) /[112]$ \\
\hline 5.82 & 0.2489280 & 34.8462 & 53.170257 & -6.09682 & $(\overline{1} 11) /[112]$ \\
\hline 5.825 & 0.2489814 & 34.8462 & 53.170257 & -6.09682 & $(111) /[112]$ \\
\hline 5.83 & 0.2491168 & 34.8462 & 53.170257 & -6.09682 & $(111) /[112]$ \\
\hline 5.835 & 0.2491695 & 34.8462 & 53.170257 & -6.09682 & $(111) /[112]$ \\
\hline 5.84 & 0.2493645 & 34.8462 & 53.170257 & -6.09682 & $(\overline{1} 11) /[112]$ \\
\hline 5.845 & 0.2494643 & 34.8462 & 53.170257 & -6.09682 & $(\overline{1} 11) /[112]$ \\
\hline 5.85 & 0.2494956 & 34.8462 & 53.170257 & -6.09682 & $(111) /[112]$ \\
\hline 5.855 & 0.2498882 & 34.8462 & 53.170257 & -6.09682 & $(\overline{1} 11) /[112]$ \\
\hline 5.86 & 0.2501108 & 34.8462 & 53.170257 & -6.09682 & $(111) /[112]$ \\
\hline 5.865 & 0.2501233 & 34.8462 & 53.170257 & -6.09682 & $(111) /[112]$ \\
\hline 5.87 & 0.2503937 & 34.8462 & 53.170257 & -6.09682 & $(\overline{1} \overline{1} 1) /[112]$ \\
\hline 5.875 & 0.2504337 & 34.8462 & 53.170257 & -6.09682 & $(\overline{1} 11) /[112]$ \\
\hline 5.88 & 0.2506551 & 34.8462 & 53.170257 & -6.09682 & $(\overline{1} 11) /[112]$ \\
\hline 5.885 & 0.2505994 & 34.8462 & 53.170257 & -6.09682 & $(\overline{1} 11) /[112]$ \\
\hline 5.89 & 0.2510399 & 34.8462 & 53.170257 & -6.09682 & $(111) /[112]$ \\
\hline 5.895 & 0.2511982 & 34.8462 & 53.170257 & -6.09682 & $(\overline{1} 11) /[112]$ \\
\hline 5.9 & 0.2516043 & 34.8462 & 53.170257 & -6.09682 & $(111) /[112]$ \\
\hline 5.905 & 0.2517063 & 34.8462 & 53.170257 & -6.09682 & $(\overline{1} 11) /[112]$ \\
\hline 5.91 & 0.2515154 & 34.8462 & 53.170257 & -6.09682 & $(111) /[112]$ \\
\hline 5.915 & 0.2510065 & 34.8462 & 53.170257 & -6.09682 & $(\overline{1} 11) /[112]$ \\
\hline 5.92 & 0.3960772 & 33.6017 & 43.829536 & -8.12909 & $(\overline{1} \overline{1} 1) /[\overline{2} 1 \overline{1}]$ \\
\hline & 0.3501054 & 31.1126 & 46.703604 & -6.09682 & $(\overline{1} 11) /[112]$ \\
\hline 5.925 & 0.7850992 & 34.8462 & 44.548053 & -6.09682 & $(\overline{1} 11) /[\overline{2} 1 \overline{1}]$ \\
\hline 5.93 & 0.8837645 & 39.8242 & 44.548053 & -6.09682 & $(\overline{1} 11) /[\overline{2} 1 \overline{1}]$ \\
\hline & 0.7988163 & 37.3352 & 38.799917 & -16.2581 & $(111) /[112]$ \\
\hline 5.935 & 0.8661576 & 36.0907 & 38.081400 & -18.2904 & $(111) /[112]$ \\
\hline & 0.8426263 & 44.8022 & 44.548053 & -6.09682 & $(\overline{1} \overline{1} 1) /[\overline{2} 1 \overline{1}]$ \\
\hline
\end{tabular}

Table 5.5: The predicted nucleation sites and slip system of dislocation with the maximum $\mathrm{N}_{m, l}$ value for the $\mathrm{Ni}(111)$ model. These results agree with the actual nucleation sites and slip systems $(\overline{1} \overline{1} 1) / \frac{1}{6}[1 \overline{2} \overline{1}]$ and $(\overline{1} \overline{1} 1) / \frac{1}{6}[\overline{2} 1 \overline{1}]$. 


\section{Appendix B: Rotation of coordinate system}

Consider two sets of rectangular Cartesian frames of reference $\mathrm{O}-x_{1} x_{2} x_{3}$ and $\mathrm{O}^{\prime}-$ $x_{1}^{\prime} x_{2}^{\prime} x_{3}^{\prime}$ in a three-dimensional space. If the origin remains fixed, and the new axes are obtained by rotating $\mathrm{O}-x_{1}, \mathrm{O}-x_{2}, \mathrm{O}-x_{3}$, then the transformation of axes is a rotation. If a point $\mathrm{P}$ has coordinates $\left(x_{1}, x_{2}, x_{3}\right),\left(x_{1}^{\prime}, x_{2}^{\prime}, x_{3}^{\prime}\right)$ relative to the old and new frames of reference in Figure 5.19, they are also the components of the radius vector $\mathrm{A}$ and $\mathrm{A}^{\prime}$.

A fact is recognized to give us immediately the law of transformation of the components of a vector in a rectangular Cartesian coordinates:

$$
\mathrm{A}_{i}^{\prime}=\beta_{i j} \mathrm{~A}_{j}
$$

In which $\beta_{i j}$ represents the cosine of the angle between the axes $\mathrm{O} x_{i}^{\prime}$ and $\mathrm{O} x_{j}$.

$$
\beta_{i j}=\operatorname{Cos}\left(\mathrm{O} x_{i}^{\prime}, \mathrm{O} x_{j}\right)=\frac{\mathrm{O} x_{i}^{\prime} \cdot \mathrm{O} x_{j}}{\left|\mathrm{O} x_{i}^{\prime}\right| \cdot\left|\mathrm{O} x_{j}\right|}
$$

For example, coordinates of atoms should be transferred from coordinate system $\mathrm{O} x_{1}:(1 \overline{1} 0), \mathrm{O} x_{2}:(11 \overline{2}), \mathrm{O} x_{3}:(111)$ to $\mathrm{O} x_{1}^{\prime}:(100), \mathrm{O} x_{2}^{\prime}:(010), \mathrm{O} x_{3}^{\prime}:(001)$ in current MD simulation, according to the Equation 5.2, matrix $\left[\beta_{i j}\right]$ can be obtained:

$$
\left[\beta_{i j}\right]=\left[\begin{array}{lll}
\frac{1}{\sqrt{2}} & \frac{-1}{\sqrt{2}} & 0 \\
\frac{1}{\sqrt{6}} & \frac{1}{\sqrt{6}} & \frac{-2}{\sqrt{6}} \\
\frac{1}{\sqrt{3}} & \frac{1}{\sqrt{3}} & \frac{1}{\sqrt{3}}
\end{array}\right]
$$

Furthermore,

$$
\left(\begin{array}{l}
1 \\
0 \\
0
\end{array}\right)=\left[\beta_{i j}\right] \frac{1}{\sqrt{2}}\left(\begin{array}{c}
1 \\
1 \\
0
\end{array}\right)=\left[\begin{array}{ccc}
\frac{1}{\sqrt{2}} & \frac{-1}{\sqrt{2}} & 0 \\
\frac{1}{\sqrt{6}} & \frac{1}{\sqrt{6}} & \frac{-2}{\sqrt{6}} \\
\frac{1}{\sqrt{3}} & \frac{1}{\sqrt{3}} & \frac{1}{\sqrt{3}}
\end{array}\right]\left(\begin{array}{c}
\frac{1}{\sqrt{2}} \\
\frac{-1}{\sqrt{2}} \\
0
\end{array}\right)
$$




$$
\begin{aligned}
& \left(\begin{array}{l}
0 \\
1 \\
0
\end{array}\right)=\left[\beta_{i j}\right] \frac{1}{\sqrt{6}}\left(\begin{array}{l}
1 \\
1 \\
2
\end{array}\right)=\left[\begin{array}{lll}
\frac{1}{\sqrt{2}} & \frac{-1}{\sqrt{2}} & 0 \\
\frac{1}{\sqrt{6}} & \frac{1}{\sqrt{6}} & \frac{-2}{\sqrt{6}} \\
\frac{1}{\sqrt{3}} & \frac{1}{\sqrt{3}} & \frac{1}{\sqrt{3}}
\end{array}\right]\left(\begin{array}{c}
\frac{1}{\sqrt{6}} \\
\frac{1}{\sqrt{6}} \\
\frac{-2}{\sqrt{6}}
\end{array}\right) \\
& \left(\begin{array}{l}
0 \\
0 \\
1
\end{array}\right)=\left[\beta_{i j}\right] \frac{1}{\sqrt{3}}\left(\begin{array}{l}
1 \\
1 \\
1
\end{array}\right)=\left[\begin{array}{lll}
\frac{1}{\sqrt{2}} & \frac{-1}{\sqrt{2}} & 0 \\
\frac{1}{\sqrt{6}} & \frac{1}{\sqrt{6}} & \frac{-2}{\sqrt{6}} \\
\frac{1}{\sqrt{3}} & \frac{1}{\sqrt{3}} & \frac{1}{\sqrt{3}}
\end{array}\right]\left(\begin{array}{c}
\frac{1}{\sqrt{3}} \\
\frac{1}{\sqrt{3}} \\
\frac{1}{\sqrt{3}}
\end{array}\right)
\end{aligned}
$$

Therefore, the law of transformation for rotation can be used to obtain the actual coordinates for all the atoms after crystal orientation.

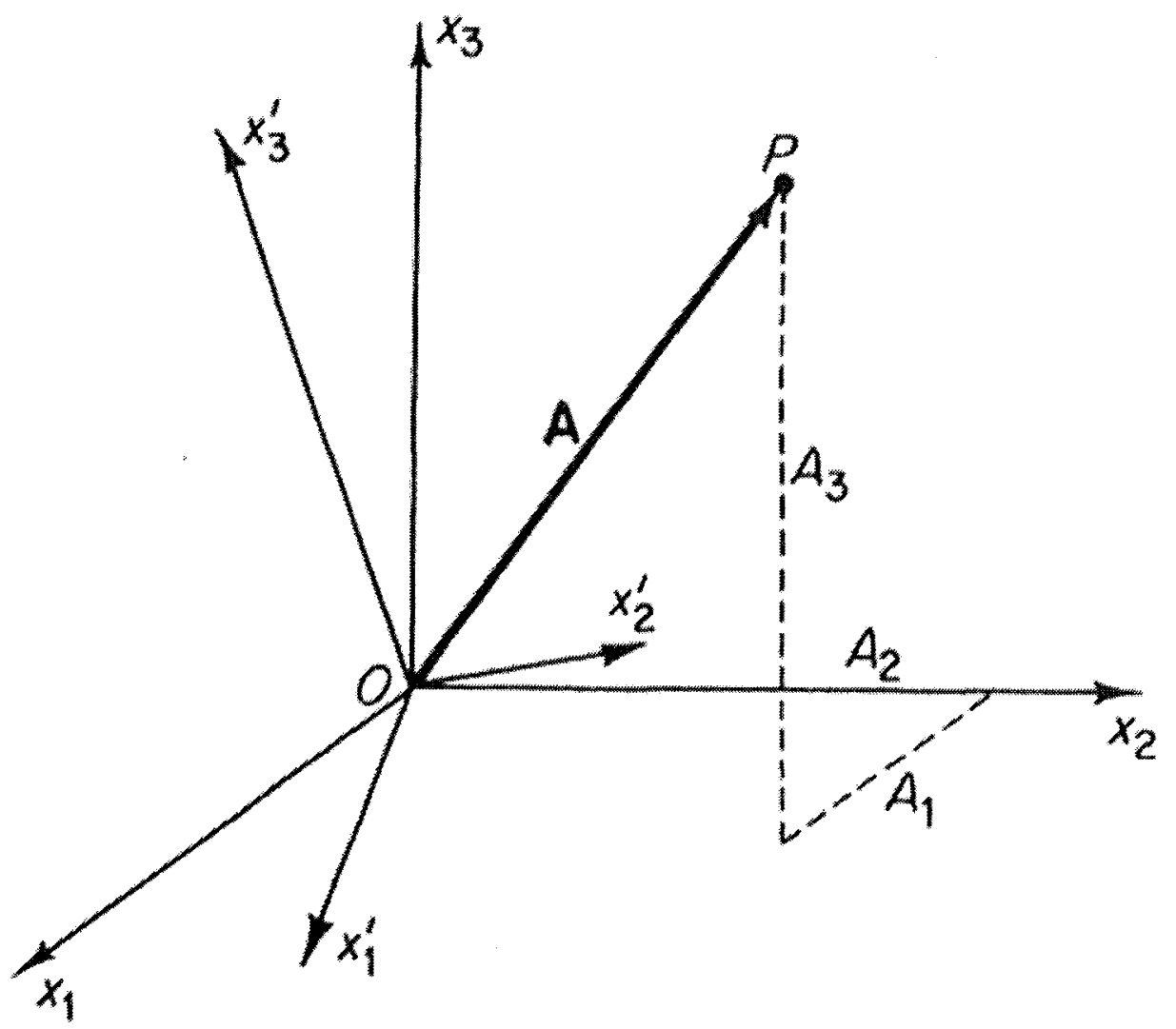

Figure 5.19: Radius vector and coordinates for 3D rotation. (Taken from [42].) 


\section{Bibliography}

[1] Ronald E. Miller and Amit Acharya. A stress-gradient based criterion for dislocation nucleation in crystals. J. Mech. Phys. Sol., 52:1507-1525, 2004.

[2] Anthony C. Fischer-Cripps. Nanoindentation. Springer, New York, NY, 2004.

[3] Andrew R. Leach. Molecular Modelling Principles and Applications. LONGMAN, 1996.

[4] O. Rodriguez de la Fuente, J. A. Zimmerman, M. A. Gonzalez, J. de la Figuera, J. C. Hamilton, W. W. Pai, and J. M. Rojo. Dislocation Emission around Nanoindentations on a (001) fcc Metal Surface Studied by STM and Atomistic Simulations. Phys. Rev. Lett., 88(3):036101, 2002.

[5] P. Grau, D. Lorenz, and A. Zeckzer. Fundamentals of Dislocation. Nucleation at Nanoindentation. Radiation Effects and Defects in Solids, 157:863-869, 2002.

[6] Krystyn J, Van Vliet, Ju Li, Ting Zhu, Sidney Yip, and Subra Suresh. Quantifying the early stages of plasticity through nanoscale experiments and simulations. Phys. Rev. B, 67(10):104105, 2003.

[7] C. L. Kelchner, S. J. Plimpton, and J. C. Hamilton. Dislocation nucleation and defect structure during surface indentation. Phys. Rev. B, 58(17):11085, 1998.

[8] H. Y. Liang, C. H. Woo, H. C. Huang, A. H. W. Ngan, and T. X. Yu. Dislocation nucleation in the initial stage during nanoindentation. Phil. Mag., 83(31-34):3609-3622, 2003. 
[9] Ju Li, Krystyn J, Van Vliet, Ting Zhu, Sidney Yip, and Subra Suresh. Atomistic mechanisms governing elastic limit and incipient plasticity in crystals. Lett. to nature., 418:307-310, 2002.

[10] Ju Li, Ting Zhu, Sidney Yip, Krystyn J. Van Vliet, and Subra Suresh. Elastic criterion for dislocation nucleation. Mat.Sci.Eng.A, 365:25-30, 2004.

[11] Ting Zhu, Ju Li, Krystyn J, Van Vliet, Shigenobu Ogata, Sidney Yip, and Subra Suresh. Predictive modeling of nanoindentation-induced homogeneous dislocation nucleation in copper. J. Mech. Phys. Sol., 52:691-724, 2004.

[12] J. de la Figuera, M. A. Gonzalez, R. Garcia-Martinez, and J. M. Rojo. STM characterization of extended dislocation configurations in Au(001). Phys. Rev. $B, 58(3): 1169(4), 1998$.

[13] F. EI Gabaly, R. Miranda, and J. de la Figuera. Properties of dislocation half loops in $\mathrm{Au}(100)$ : structure, formation energy, and diffusion barrier. Phys. Rev. $B, 70(1): 012102(4), 2004$.

[14] J. D. Kiely and J. E. Houston. Nanomechanical properties of Au (111),(001), and (110) surfaces. Phys. Rev. B, 57(19):12588(7), 1998.

[15] J. A. Zimmerman, C. L. Kelchner, P. A. Klein, J. C. Hamilton, and S. M. Foiles. Surface Step Effects on Nanoindentation. Phys. Rev. Lett., 87(16):165507, 2001.

[16] V. Yamakov, D. Wolf, S. R. Phillpot M. Salazar, and H. Gleiter. Length-scale Effects in the Nucleation of Extended Dislocations in Nanocrystalline Al by Molecular-Dynamics Simulation. Acta Mat., 49:2713-2722, 2001.

[17] Duesbery M. S. and Vitek V. Yielding of Steel Sheets Containing Slits. Acta Mat., 46:1481-1492, 1998. 
[18] Gouldstone A., Van Vliet K. J., and Suresh S. Nanoindentation: simulation of defect nucleation in a crystal. Nature, 411:656, 2001.

[19] Rice J. R. and Beltz G. E. Local Structural Fluctuations in Amorphous and Liquid Metals: A Simple Theory of the Glass Transition. J. Mech. Phys. Sol., 42:333-360, 1994.

[20] Shenoy V. B., Phillips R., and Tadmor E. B. Nucleation of dislocations beneath a plane strain indenter. J. Mech. Phys. Sol., 48:649-673, 2000.

[21] E. B. Tadmor and S. Hai. A Peierls Criterion for the Onset of Deformation Twinning at Crack Tips. J. Mech. Phys. Sol., 51:765-793, 2003.

[22] D. Hull and D. J. Bacon. Introduction to Dislocations. Butterworth-Heinemann, Oxford, 2001.

[23] James F. Shackelford. Introduction to Materials science for engineers. PRENTICE-HALL, 1996.

[24] W. T. Read. Dislocations in Crystals. McGraw Hill, 1953.

[25] J. Chen, W. Wang, L. H. Qian, and K. Lu. Critical shear stress for onset of plasticity in a nanocrystalline $\mathrm{Cu}$ determined by using nanoindentaion. Scripta Materialia, 49:645-650, 2003.

[26] J. D. Kiely, R. Q. Hwang, and J. E. Houston. Effect of Surface Steps on the Plastic Threshold in Nanoindentation. Phys. Rev. Lett., 81(20):4424, 1998.

[27] C. F. Robertson and M. C. Fivel. A study of the submicron indent-induced plastic deformation. J.Mat.Res, 14(6):2251-2258, 1999.

[28] E. T. Lilleodden, J. A. Zimmerman, S. M. Foiles, and W. D. Nix. Atomistic simulations of elastic deformation and dislocation nucleation during nanoindentation. J. Mech. Phys. Sol., 51:901-920, 2003. 
[29] E. B. Tadmor, R. Miller, and R. Phillips. Nanoindentation and incipient plasticity. J.Mat.Res, 14(6):2233-2250, 1999.

[30] M. Rieth and W. Schommers. Handbook of Theoretical and Computational Nanotechnology. American Scientific, CA, 2005.

[31] S. M. Foiles, M. I. Baskes, and M. S. Daw. Embedded-atom-method functions for the fcc metals $\mathrm{Cu}, \mathrm{Ag}, \mathrm{Au}, \mathrm{Ni}, \mathrm{Pd}, \mathrm{Pt}$ and their alloys. Phys. Rev. B, 33(12), 1986.

[32] Murray S. Daw and M. I. Baskes. Embedded-atom method: Derivation and application to impurities, surfaces, and other defects in metals. Phys. Rev. B, 29(12):6443-6453, 1984.

[33] J. Li and S. Yip. Atomistic Measures of Materials Strength. Computer modeling in engineering Sciences, 3(2):219-227, 2002.

[34] L. E. Shilkrot, R. Miller, and W. A. Curtin. Coupled Atomistic and Discrete Dislocation Plasticity. Phys. Rev. Lett., 89(2):025501, 2002.

[35] M. S. Daw and S. M. Foiles. Dynamo version 8.7. FORTRAN code, 1994.

[36] Adeyemi J. Akangbe. M.App.Sc thesis. Carleton University, 2003.

[37] C. Zuppa and A. Cardona. A collocation meshless method based on local optimal point interpolation. Int.J.Numer.Meth.Engng, 57:509-536, 2003.

[38] Ron. E. Miller. PhD thesis. Brown University, 1997.

[39] J.Y. Li, S.W. Luo, and Y.J. Qi. Approximation of Function by Adaptively Growing Radial Basis Function Neural Networks. ESANN'2003 proceedings - European Symposium on Artificial Neural Networks Bruges, pages 113-118, 2003. 
[40] K. L. Johnson. Contact mechanics. Cambridge, New York, NY, 1985.

[41] Anil Gannepalli and Surya K. Mallapragada. Atomistic studies of defect nucleation during nanoindentation of $\mathrm{Au}(001)$. Phys. Rev. B, 66(10):104103, 2002.

[42] Y. C. Fung. A First Course In Continuum Mechanics. Prentice-Hall, Inc., Englewood Cliffs, New Jersey, 1977. 